\title{
Mechanistic Plasticity and Molecular Recognition: The Structural Biology of the MAP Kinase Interacting Kinases 1 and 2, the NAD Synthetase and the Zinc Finger Associated Domain
}

\author{
PhD Thesis \\ In partial fulfillment of the requirements for the degree of \\ Doctor of Philosophy (Ph.D.) / Doctor rerum naturalium (Dr. rer. nat.) \\ in the Molecular Biology Program \\ at the Georg-August University Göttingen, \\ Faculty of Biology
}

Submitted by

RALF JAUCH

Born on the 28.06.1977 in Weida (Thüringen)

Göttingen, 2005 


\section{Affidavit}

Here I declare that my Ph.D. thesis entitled "Mechanistic Plasticity and Molecular Recognition: The Structural Biology of the MAP Kinase Interacting Kinases 1 and 2, the NAD Synthetase and the Zinc Finger Associated Domain" has been written independently with no other sources and aids than quoted.

Ralf Jauch

Göttingen, 27.09.2005 


\section{Table of Contents}

$\begin{array}{lr}\text { Abstract } & 4\end{array}$

Introduction $\quad 5$

Scope and Motivation $\quad 5$

$\begin{array}{ll}\text { Tuning Cells with Phosphoryl Switches } & 6\end{array}$

The Making of a Protein Kinase follows a Conserved Construction Plan 7

Protein Kinase Function 10

The Regulation of Protein Kinases is Diverse 11

The Subfamily of MAPK Interacting Kinases $\quad 13$

Chapter I: Crystal Structures of the Mnk2 Kinase Domain Reveal an Inhibitory Conformation and a Zinc-Binding Site $\quad 16$

Chapter II: The MAPK Interacting Kinase (Mnk) Subfamily is Regulated via a Novel Autoinhibitory Mechanism

Supplementary Chapter III: Structures of Escherichia coli NAD Synthetase with substrates and products reveal mechanistic rearrangements

Supplementary Chapter IV: The zinc finger-associated domain of the Drosophila transcription factor Grauzone is a novel zinc-coordinating protein-protein interaction module

General Discussion

Zinc Coordinating Protein Modules and Intermolecular Recognition

Mechanistic Plasticity of Biological Catalysts: Lessons from Mnk1/2 and NADS

The Structures of Mnk1 and Mnk2 Reveal a Novel Mechanism of Protein Kinase Regulation

The Structures of ZAD, NADS and Mnk1/2 are Relevant for Structure Guided Drug Design

Bibliography

Abbreviations

Acknowledgements

Curriculum Vitae 


\section{Abstract}

In this study a set of four different proteins has been examined structurally and functionally. The core results embody the structural analysis of the catalytic domains of the mitogen activated protein kinase (MAPK) interacting kinases (Mnk) 1 and 2. These proteins are Ser/Thr protein kinases which target the translation initiation machinery in response to activation by MAPK cascades. Despite a highly similar primary sequence the crystal structures of Mnk1 and Mnk2 exhibit pronounced differences in structural modules which are required for enzymatic activity. Both proteins were found to adopt a conformation that is incompatible with productive binding to either ATP or the peptide substrate. The structural snapshots obtained from Mnk1, Mnk2 and Mnk2 ${ }^{\mathrm{D} 228 \mathrm{G}}$, a Mnk2 active site mutant, in combination with activity assays enable to derive a model for the functional dynamics common to Mnk subfamily members. The model predicts that activation of these protein kinases is guided by pronounced rearrangements of the activation segment. The results indicate that the regulation of Mnks involves autoinhibition mediated by the structurally reorganized activation segment and suggests strategies for inhibitor development.

A second example highlighting the structural rearrangements which accompany the activity cycle of an enzyme is the Escherischia coli nicotinamide adenosine dinucleotide synthetase (ecoNADS). An ensemble of five crystal structures of this enzyme was analyzed. They show that the structural rearrangements of ecoNADS are dependent on the presence of its substrates and products. The results allow defining a catalytic state and a substrate/product exchange state of the enzyme.

Finally, the insect-specific zinc-finger associated domain (ZAD) was investigated. The ZAD constitutes a highly expanded sequence motif in Drosophila. Biochemical and structural data revealed that the ZAD of the Drosophila transcription factor Grauzone adopts a novel zinc coordinating fold. This fold bears a fingerprint of hydrophobic residues that facilitate homodimerisation in an antiparallel fashion. The results show that the ZAD constitutes a protein-protein recognition module which participates in the transcriptional regulation in specific phylogenetic lineages. 


\section{Introduction}

\section{Scope and Motivation}

Richard Feynman, who received the Nobel Prize in physics in 1965, once pointed out that: "...everything that living things do can be understood in terms of jigglings and wigglings of atoms" (Feynman et al., 1963). Although this reductionist view may not be common sense, there is no doubt that structural biology is concerned with one of the most fundamental aspect of biology - how the physical shape of biological macromolecules determines their function. The present work aimed at the elucidation of dynamical features of biological catalysts and addresses the questions how those features guide the enzymatic reaction and regulate the activity. Furthermore, it was attempted to study the molecular recognition on the proteinprotein as well as on the protein-ligand level. To this end, the structure and function of three nucleotide utilizing enzymes, two closely related protein kinases MAP kinase interacting kinases (Mnk) 1 and 2 (Chapters $I$ and II) and the Escherischia coli nicotinamide adenosine dinucleotide synthetase (ecoNADS) (Supplementary Chapter III) were investigated. In addition, the zinc-finger associated domain (ZAD) of the Drosophila transcription factor Grauzone was studied (Supplementary Chapter IV).

The studies on Mnk1 and Mnk2 were initiated to define the structural basis for Mnk regulation and to propose novel strategies for protein kinase inhibitor development. The investigation of various structures of the ecoNADS was considered to provide an instructive complementation of studies on the mechanistic dynamics of nucleotide utilizing enzymes. In addition, the bacterial NADS has recently been highlighted as promising target to develop anti-anthrax drugs (Sutherland, 2003). The phylogenetic comparison to the Bacillus subtilis NADS ( Rizzi et al., 1996 ;Rizzi et al., 1998), which is highly similar to the enzyme of the pathogen Bacillus anthracis, was therefore expected to reveal intricate details within the substrate recognition site which are relevant for designing antimicrobial agents.

The investigation of the ZAD extended the in silico characterization of this insect specific protein module (Chung et al., 2002). The ZAD constitutes and abundant sequence motif that is expanded in insect genomes and shares no sequence homology to previously 
determined structures. By characterizing ZAD it was attempted to clarify the functional roles of this protein module and to provide a structural view on a potential insecticide target.

By the time of completion of this $\mathrm{PhD}$ thesis, three manuscripts presented herein have been published (Supplementary Chapters III and IV) or are in press (Chapter I; scheduled for publication October 2005) in peer-review journals. The manuscript presented in Chapter II will be submitted for publication in due course. Copies of the manuscripts can be found in the corresponding chapters. The potential application of the investigated structures for drug design efforts has been protected by patent application in the case of the ZAD (European Patent 03017076) and Mnk1/2 (European Patent 050198899). The study on the Mnk subfamily as a prototype variation of eukaryotic protein kinases (PKs) has been the main focus of this work and has therefore received the largest overall attention. Hence, the Chapters I and II containing the studies on Mnk1 and Mnk2 constitute the core of this $\mathrm{PhD}$ thesis, whereas the work on NADS and ZAD is considered as a supplementation that frames the core project. To highlight this weightening, the chapters discussing NADS and ZAD are denoted as Supplementary Chapters $I I I$ and $I V$. Priorities given within the introduction and the discussion were adjusted accordingly.

\section{Tuning Cells with Phosphoryl Switches}

Reversible phosphorylation acts as a molecular switch which regulates a multitude of biological activities (Krebs, 1983; Pawson and Scott, 2005). Pioneering work that demonstrated this profound biological insight has been carried out by Edwin G. Krebs, Edmond H. Fischer and Earl Sutherland in the 1950s by studying the rabbit muscle phosphorylase (Fischer and Krebs, 1955; Sutherland and Wosilait, 1955; Krebs and Fischer, 1956). Subsequent studies lead to the discovery of PKs as the molecular players behind those events (Krebs et al., 1958). PKs constitute a class of enzymes which catalyze the transfer of the terminal phosphat group of ATP or related nucleotides onto the hydroxyl group of the ami- 


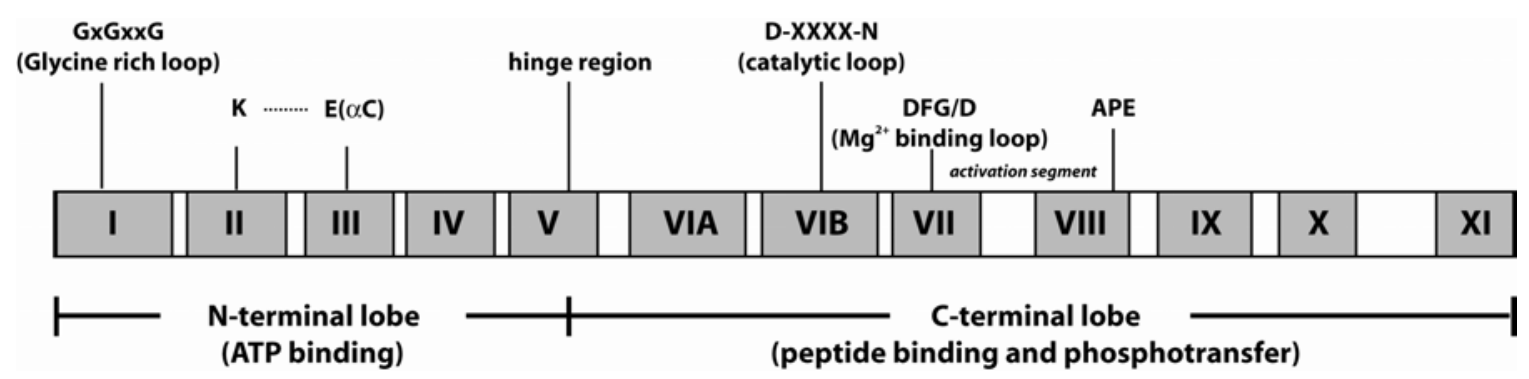

Figure 1: Structural and functional organization of protein kinase domains.

The 11 conserved subdomains of a protein kinase are indicated by roman numbers (I to XI; Hanks, 1988; Hanks et al., 2003). Functional sites are labeled and conserved amino acids motifs are indicated using the one letter code. X means any amino acid.

no acid residues serine, threonine or tyrosine within substrate proteins. This covalent modification may lead to an alteration of the activity, stability or subcellular localization of the affected proteins (Krebs, 1983; Pawson and Scott, 2005).

Protein kinases are often activated by extracellular stimuli. Thus, they enable cells to adapt their physiology in response to external signals, e.g. during cell-cell communication. Genome sequencing projects underlined the significance of the PKs by identifying more than 500 members of this gene family encoded by the human genome (Manning et al., 2002). This large number groups PKs among the most abundant gene families only topped in number by genes encoding the $\mathrm{C} 2 \mathrm{H} 2$ Zinc-finger and the immunoglobulins (Lander et al., 2001).

\section{The Making of a Protein Kinase Follows a Conserved Construction Plan}

The investigation of an increasing number of PKs led to the recognition of common features with a putative role in the catalytic reaction. The functional domain, which spans 250 to 300 amino acids, has been defined and was further divided into 11 conserved subdomains (I to XI form N-to C-terminus; Figure 1; Hanks et al., 1988). 

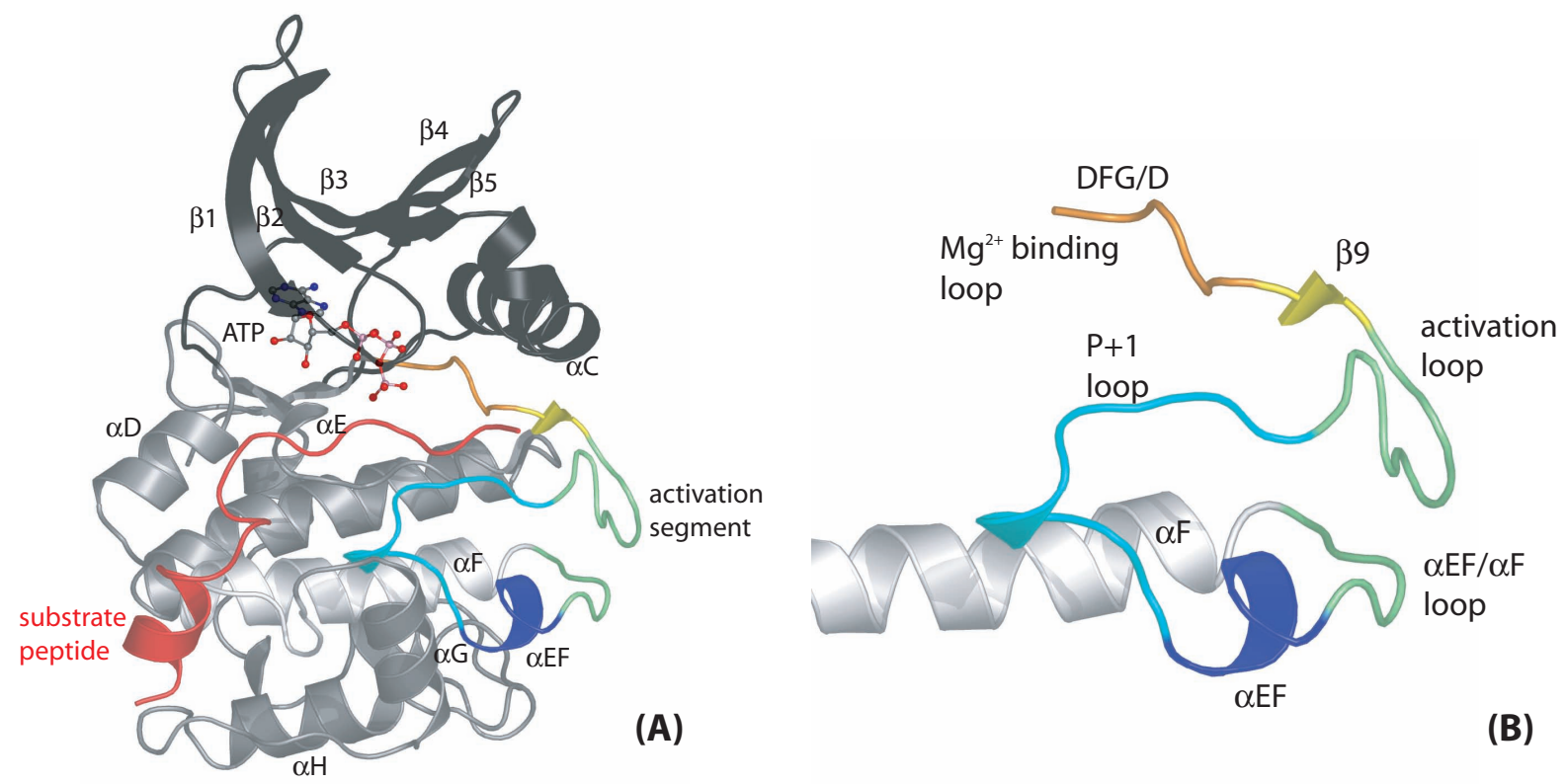

(B)

Figure 2: The topology of a prototype protein kinase and the nomenclature of the activation segment.

(A) The cAMP dependent protein kinase (PKA) in complex with an ATP molecule (ball-and-stick-representation) and a peptide inhibitor (PDB-id: 1atp). The peptide inhibitor mimics a substrate peptide (red). The N-terminal lobe is depicted in black and the $\mathrm{C}$-terminal lobe in gray. The secondary structure is labeled according to standard conventions and used henceforth in the text accordingly. (B) Enlarged depiction of the activation segment and $\alpha F$ of PKA in the same orientation as in (A). The activation segment in (B) represents a prototype activation segment in its active configuration. Structural elements of the activation segment discussed in the text are indicated.

Certain amino acid motifs have been found in the vast majority of protein kinases such as the Gly-X-Gly-X-X-Gly (X corresponds to any amino acid) motif of subdomain I, the Lys of subdomain II, an Asp-X-X-X-X-Asn motif of subdomain VI and an Asp-Phe-Gly (DFG) motif of subdomain VII (Hanks et al., 1988). The region between the DFG motif and the AspPro-Glu (APE) motif, the latter denotes a conserved tripeptide of subdomain VIII, often bears residues which are targeted for activating phosphorylation by upstream kinases and has thus been termed the 'activation segment' (Johnson et al., 1996; Nolen et al., 2004).

The first crystal structure of a PK had been published in 1991 and revolutionized the understanding of PK function (Knighton et al., 1991a). The cAMP dependent PK (PKA) in complex with $\mathrm{Mg}^{2+}$-ATP and an inhibitory peptide unraveled the overall fold as well as the modes of nucleotide and peptide recognition of a prototype PK (Knighton et al., 1991a; Knighton et al., 1991b; Figure 2). Subsequent studies demonstrated that the overall architectural makeup of PKs is conserved (Taylor and Radzio-Andzelm, 1994; Johnson et al., 1996; Huse and Kuriyan, 2002). In short, PK domains (PKDs) fold into two structural modules which are termed the 'N-terminal lobe' (subdomain I to $\mathrm{V}$ ) and the 'C-terminal lobe' 
(subdomain $\mathrm{V}$ to $\mathrm{XI}$ ), respectively. This makeup leads to a characteristic bilobal architecture of the PKD. The ATP molecule is sandwiched in a deep groove between both lobes termed the interlobal cleft (Figure 2A). The N-terminal lobe is composed of a twisted $\beta$-sheet comprising five antiparallel strands $(\beta 1-\beta 5)$ and the helix $\alpha \mathrm{C}$. Certain PKs such as PKA contain more $\alpha$ helices within the $\mathrm{N}$-terminal lobe of which only $\alpha \mathrm{C}$ is conserved throughout the PK superfamily. The linker between the two lobes is referred to as the 'hinge' region. The hinge region participates in the interaction with the ATP molecule through main-chain H-bonds with the N1 and 6-amino group of the adenine. The C-terminal lobe consists largely of a $\alpha$-helical bundle $(\alpha \mathrm{D}-\alpha \mathrm{H})$ and bears the structural elements required for peptide binding and phosphoryl transfer. One of these elements is the activation segment (Figure 2B). The activation segment is a highly dynamic entity and its conformation is often associated with the catalytic activity. The activation segment can be further subdivided into structurally and functionally distinct components. It harbors (in N- to C-terminal direction) the magnesium binding DFG/D motif, the activation loop which is often phosphorylated by upstream PKs, the P+1 loop and the short helix $\alpha E F$ (Nolen et al., 2004). The $\alpha E F / \alpha F$ loop constitutes the C-terminal 'anchor' of the activation segment and connects it to helix $\alpha \mathrm{F}$ (Figure 2B). The structural elements downstream of $\alpha \mathrm{F}$ lack a functionally copious annotation. However, recent reports suggest that helices $\alpha \mathrm{G}$ and $\alpha \mathrm{H}$ at the C-terminal end of the PKD provide an important scaffold required for the interaction with substrate and/or regulatory proteins (Kannan and Neuwald, 2005; Kim et al., 2005). 


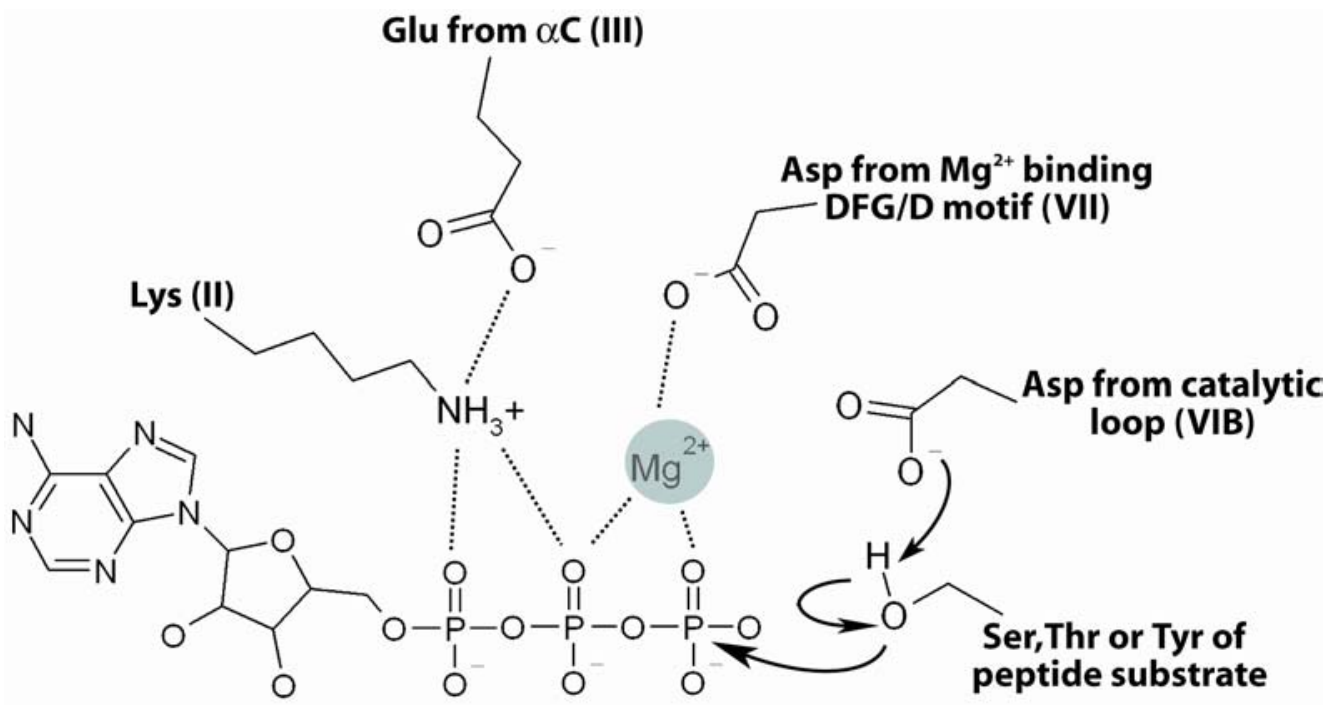

Figure 3: Active site residues and reaction mechanism of a protein kinase.

An ATP molecule and the side chains of amino acids with immediate relevance for the phosphoryl transfer are shown. The reaction mechanism depicted here assumes a general base catalysis (as indicated by the arrows). However, the actual biochemical events are still under debate (Adams, 2001; Hart et al., 1998). Only the nonhydrogen and non-carbon atoms are shown. The subdomains from which the amino acid residues originate are shown in brackets.

\section{Protein Kinase Function}

PKs facilitate the phosphoryl transfer through accurate positioning of the reactants by active site elements. These elements often constitute highly conserved entities (Taylor and Radzio-Andzelm, 1994; Figure 3). The phosphate groups of ATP are held in place by the Glyrich loop (Gly-X-Gly-X-X-Gly motif; subdomain I) and the invariant Lys from subdomain II. This Lys is in turn positioned by an invariant Glu which emanates from helix $\alpha \mathrm{C}$ and forms a Lys-Glu ion pair found in all active PKs. The terminal phosphate group of ATP is polarized and thereby activated, by a catalytic magnesium ion which is coordinated by the first Asp of the DFG/D motif (Adams, 2001). As a consequence, the $\gamma$-phosphate group becomes positioned for the nucleophilic attack by the hydroxyl group of the substrate peptide. The latter reaction is further potentiated by the catalytic Asp emanating from the catalytic loop (C-loop; corresponding to the conserved Asp-X-X-X-X-Asn motif of subdomain VI). The catalytic Asp has been proposed to either act as a base catalyst by accepting a proton from the substrate or as 
stabilizer of a protonated transition state of the $\gamma$-phosphate group (Adams, 2001; Hart et al., 1998; Figure 3).

In order to enable the phosphoryl transfer, the substrate peptide has to be docked to the active site with the phosphate receiving group in close proximity to the terminal phosphate of ATP ( Hubbard, 1997; Brown et al., 1999; Yang et al., 2002). This positioning is achieved through contributions of residues from the C-loop as well as from the $\mathrm{P}+1$ loop located within the activation segment. The $\mathrm{P}+1$ loop received its designation because it binds the residue of the peptide substrate which is located downstream of the phosphorylation site ( $\mathrm{P} 0$-site; residues upstream are designated $\mathrm{P}-1, \mathrm{P}-2$ and so forth; residues downstream $\mathrm{P}+1, \mathrm{P}+2$ etc) (Knighton et al., 1991a). Therefore, the $\mathrm{P}+1$ loop plays an important role in conferring substrate specificity (Hanks, 2003). The discrimination between protein Ser/Thr and Tyr kinases, for example, is essentially achieved through a single amino acid residue from the $\mathrm{P}+1$ loop. In the case of a Ser/Thr kinase, the P+1 loop bears either a Ser or a Thr which forms hydrogen bonds with the catalytic Asp (Knighton et al., 1991a; Lowe et al., 1997; Yang et al., 2002). In a Tyr kinase, however, the Ser/Thr is substituted by a Pro which engages in a stacking interaction with the phenol of the substrate tyrosine and thereby positions this amino acid residue for catalysis (Hubbard, 1997).

\section{The Regulation of Protein Kinases is Diverse}

Despite the conserved architecture of their catalytic domains, individual PKs often function in distinct physiological contexts and are regulated by a number of different mechanisms. PK activity is adjusted, for example, by signaling molecules such as cyclic nucleotides (Kim et al., 2005) and $\mathrm{Ca}^{2+} /$ Calmodulin (Goldberg et al., 1996), by covalent modifications such as phosphorylation (Hubbard, 1997) or by interactions with regulatory subunits and regulatory domains (Jeffrey et al., 1995; Kobe et al., 1996). In many cases, PK regulation involves a combination of these mechanisms. The biochemical events accompanying PK activation induce intricate conformational changes within the catalytic domain which leads to a positioning of active site elements. This way, recognition sites for ATP or substrate peptides are made accessible. However, there is no stereotypical mode of kinase regulation and the structural changes accompanying PK activation are highly diverse 
(see, for example, p38 (Bellon et al., 1999); cyclin dependent kinase 2 (CDK2; Jeffrey et al., 1995), Twitchin (Kobe et al., 1996); CAMKI (Goldberg et al., 1996); insulin receptor kinase (IRK; Hubbard, 1997); c-Abl (Nagar et al., 2003); FLT3 (Griffith et al., 2004)). The understanding of the regulation of individual PKs does therefore require a delineation of mechanistic events at the atomic level.

The impact of structure-function studies on PKs is underlined by the fact that deregulation of PK activities is a major cause of human diseases including cancer, inflammation and arthritis (Cohen, 2002; Vieth et al., 2005). Hence, the investigation of the structural basis for PK regulation assists in diagnosing, understanding and readjusting PK malfunction, for example, by rationalizing efforts to design specific PK inhibitors (Gill et al., 2005). The present work elucidates regulatory principles of the Mnk subfamily of PKs and proposes how these principles can be applied for therapeutic interventions.
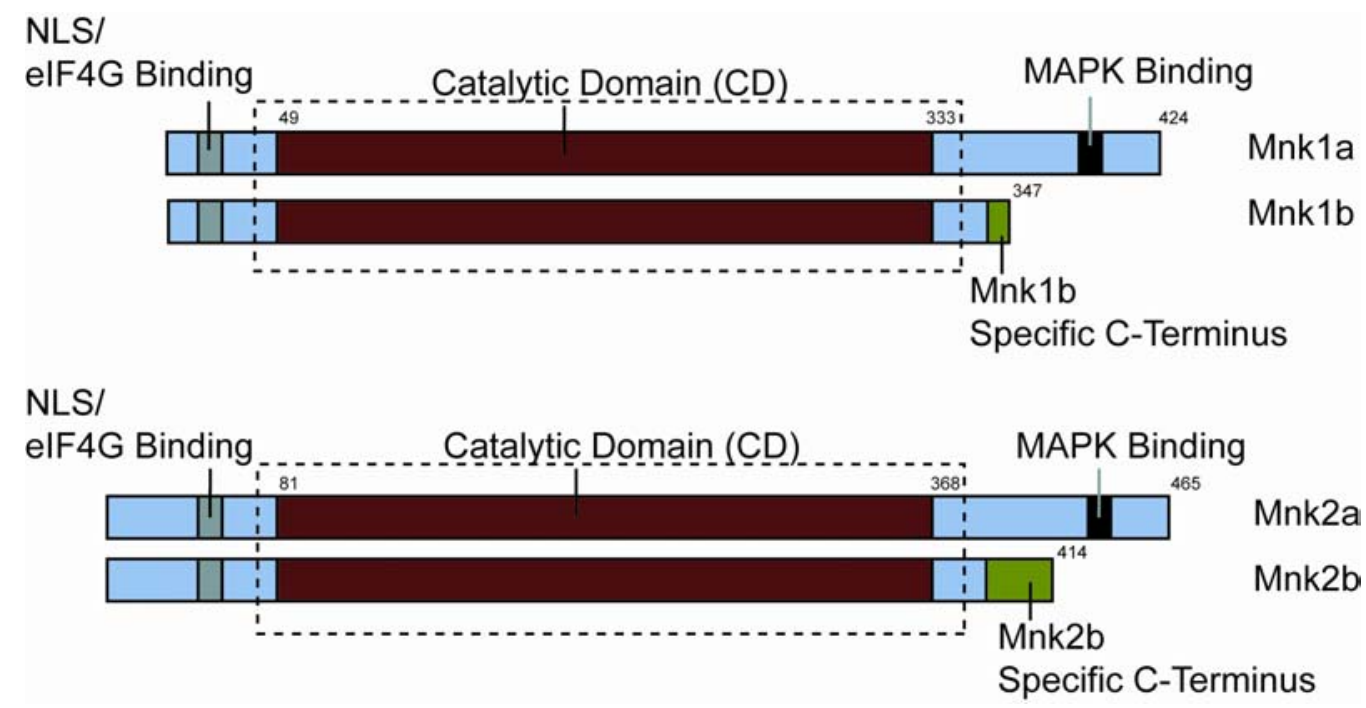

Figure 4: Organization of the human Mnk proteins .

Schematic depiction of the two human Mnk isoforms indicating the arrangement of functional domains (as labeled). The region investigated herein (Mnk1/2 kinase region, Mnk1/2-KR) is boxed with dashed lines. Alternative splicing does neither affect the N-terminus nor the kinase domain. NLS - nuclear localization signal. eIF4G - eukaryotic initiation factor $4 \mathrm{G}$, the scaffolding protein of the translation initiation complex which binds Mnk1 and Mnk2 (Pyronnet et al., 1999; Scheper et al., 2001). 


\section{The Subfamily of MAPK Interacting Kinases}

The analysis of the phylogenetic tree of PKs led to the definition of nine major groups termed after prominent group members (Manning et al., 2002). These groups were further subdivided into families and subfamilies (www.kinase.com). The $\mathrm{Ca}^{2+} / \mathrm{Calmodulin}$ modulated protein kinase (CAMK) group comprises 80 members including the name-giving CAMK family of PKs, the phosphorylase kinase family and MAP kinase associated protein kinase (MAPKAP) family (Hanks, 2003).

The Mnks constitute a subfamily belonging to the MAPKAPs encoded by two genes within the human genome (mnkl and mnk2). Both Mnk isoforms are alternatively spliced leading to a total of four proteins products. The resulting proteins are termed Mnk1a, Mnk1b, Mnk2a and Mnk2b and range from 347 to 465 amino acid residues ( Slentz-Kesler et al., 2000; Scheper et al., 2003; O'Loghlen et al., 2004; Figure 4). The differential splicing affects a region C-terminal of the central kinase domain which bears both a Crm-1 type nuclear export signal (Fornerod et al., 1997) and a MAPK binding motif. This way, the subcellular localization and the basal activity of the corresponding proteins is altered (O'Loghlen et al., 2004; Parra-Palau et al., 2003). In contrast, the region N-terminal of the PK domain is common to all four splice-products (Scheper and Proud, 2002). This region bears a basic sequence which functions as a nuclear localization signal and a binding region for the eukaryotic initiation factor 4G (eIF4G; Scheper and Proud, 2002). The catalytic PKDs of Mnk1 and Mnk2 locates to the center of the enzymes and spans their largest portion. The PKD of both isoforms exhibits an amino acid identity of $78 \%$ suggesting similar substrate specificities and regulatory principles.

Mnks are downstream components of intracellular signal transduction pathways (Duncan et al., 2005). They are activated in response to phosphorylation by the MAP kinases ERK1/2 and p38, which are activated upon cellular stress, cytokine or mitogen induced signaling cascades (Fukunaga and Hunter, 1997; Waskiewicz et al., 1997; Scheper et al., 2001). The hitherto most intensively studied Mnk substrate is the eukaryotic initiation factor 4E (eIF4E) (Scheper and Proud, 2002). Mnks have been shown to phosphorylate eIF4E at a conserved serine close to its substrate binding region in vitro and in vivo ( Waskiewicz et al., 1997; Waskiewicz et al., 1999; Ueda et al., 2004). 
The Mnk substrate eIF4E is a component of the trimeric eIF4F complex which is required to initiate the translation of most eukaryotic mRNAs. The eIF4F complex contains, in addition, the DEAD-box RNA helicase eIF4A and the scaffold protein eIF4G. eIF4E is required to link this complex to the mRNA's 5'-terminus by physically binding to the $\mathrm{m}^{7} \mathrm{GpppN}$ cap structure $\left(\mathrm{m}^{7} \mathrm{G}\right.$ represents 7-methylguanylate; $\mathrm{p}$ represents phosphate groups and $\mathrm{N}$ represents any base; Marcotrigiano et al., 1997; Preiss and Hentze, 2003). A cap-bound eIF4F complex is essential for the recruitment of the $43 \mathrm{~S}$ pre-initiation complex including the small ribosomal subunit and the initiator tRNA to the mRNA ( Sachs, 2000; Preiss and Hentze, 2003; Proud, 2005). Since the initiation of translation is tightly controlled, e.g. by posttranslational modifications of initiation factors, Mnks could potentially function as regulators of gene expression on the level of mRNA translation (Gebauer and Hentze, 2004). It has indeed been demonstrated that phosphorylation of eIF4E reduces the binding affinity to capped mRNA in vitro (Scheper et al., 2002; Zuberek et al., 2003). However, the physiological effects of eIF4E phosphorylation on mRNA translation are under debate, but it seems that the overall protein synthesis is not affected by eIF4E phosphorylation (McKendrick et al., 2001; Morley and Naegele, 2002; Ueda et al., 2004). Instead, several reports suggest that effects of Mnk activity is restricted to regulate the translation of specific mRNAs (Nikolcheva et al., 2002; Walsh and Mohr, 2004; Buxade et al., 2005). It has been suggested that transcripts with a complex 5'-untranslated region (UTR) structure are susceptible for Mnk-mediated regulatory mechanisms (Sonenberg and Gingras, 1998).

A recent study indicates an impact of Mnks on mRNA translation which is independent of eIF4E (Buxade et al., 2005). In this study, it was shown that Mnk1 phosphorylates the heterogenous nuclear ribonucleoprotein A1 (hnRNP A1). hnRNP A1 binds AU rich sequences within the 3'-UTR structures of many eukaryotic mRNAs and thereby affects various aspects of mRNA regulation including translation, splicing and subcellular localization (Allemand et al., 2005). As a result of Mnk1 mediated phosphorylation of hnRNP A1, the translation of the tumor necrosis factor alpha (TNF- $\alpha$ ) was downregulated (Buxade et al., 2005). Hence, Mnks target not only eIF4E but also other components of the translation initiation machinery. This way, it appears to regulate the translation of a distinct set of transcripts rather than translation in general.

The recent observation that mice homozygous for a targeted disruption of either one or both Mnk genes show no signs of abnormal development is in line with the conclusion that 
Mnks are not essential for overall protein synthesis (Ueda et al., 2004). However, Drosophila flies, which have lost the single functional Mnk1/2 homolog, termed Lk6 develop a dietdependent growth phenotype (Reiling et al., 2005): The lk6 gene is dispensable when the flies were kept on a high protein diet, whereas a significant and eIF4E-mediated growth reduction has been observed with $L k 6$ mutant flies raised on food low in amino acids (Arquier et al., 2005; Reiling et al., 2005). Taken together, the observations of a diet-dependence of Lk6 activity in the fly (Reiling et al., 2005), the indication of Mnk substrates in addition to eIF4E (Hefner et al., 2000; Buxade et al., 2005) as well as the specific role of Mnks in viral translation (Walsh and Mohr, 2004) and in the translation of TNF- $\alpha$ (Buxade et al., 2005) suggest intricate not yet unraveled mechanisms of Mnk action with relevance for human health. Here I will present the crystal structures and biochemical data of the catalytic domains of the two human subfamily members, Mnk1 and Mnk2, and of a Mnk2 active site mutant. The results show that Mnks represent a prototype variation of PKs. The structures provide evidence that Mnks evolved a novel autoinhibitory mechanism. This mechanism is guided by a structurally rearranged activation segment (Chapter I, II). 


\section{Chapter I: Crystal Structures of the Mnk2 Kinase Domain Reveal an Inhibitory Conformation and a Zinc-Binding Site}

\section{Citation:}

Jauch, R., Jäkel, S., Netter, C., Schreiter, K., Aicher, B., Jäckle, H., and Wahl, M. (2005). Crystal Structures of the Mnk2 Kinase Domain Reveal an Inhibitory Conformation and a ZincBinding Site. Structure. Scheduled for publication October 2005

\section{Statement of Contribution:}

crystallization, data collection, structure solution, structure refinement, model building, structure analysis, examination of and comparison to available protein kinase structures, analytical gel filtration, light scattering, ATP binding assay, manuscript preparation 


\title{
Crystal Structures of the Mnk2 Kinase Domain Reveal an Inhibitory Conformation and a Zinc-Binding Site
}

\author{
Ralf Jauch $^{1}$, Stefan Jäkel ${ }^{2}$, Catharina Netter ${ }^{3}$, Kay Schreiter ${ }^{2}$, Babette Aicher $^{2}$, Herbert Jäckle ${ }^{1}$ \\ and Markus C. Wahl ${ }^{3 *}$
}

Max-Planck-Institut für Biophysikalische Chemie

${ }^{1}$ Abteilung Molekulare Entwicklungsbiologie

${ }^{3}$ Abteilung Zelluläre Biochemie/Röntgenkristallographie

Am Faßberg 11

D-37077 Göttingen, Germany

${ }^{2}$ Develogen AG

Rudolf-Wissell-Str. 28

D-37079 Göttingen, Germany

Human mitogen-activated protein kinases (MAPK)-interacting kinases 1 and 2 (Mnk1 and Mnk2) target the translational machinery by phosphorylation of the eukaryotic initiation factor 4E (eIF4E). Here we present the $2.1 \AA$ crystal structure of a nonphosphorylated Mnk2 fragment that encompasses the kinase domain. The results show unique Mnk-specific features such as a zinc-binding motif and an atypical open conformation of the activation segment. In addition, the ATP binding pocket contains an Asp-Phe-Asp (DFD) in place of the canonical magnesium-binding Asp-Phe-Gly (DFG) motif. The phenylalanine of this motif sticks into the ATP binding pocket and blocks ATP binding as observed with inhibitor-bound and thus inactive p38 kinase. Replacement of the DFD by the canonical DFG motif affects the conformation of Mnk2, but not ATP binding and kinase activity. The results suggest that the ATP binding pocket and the activation segment of Mnk2 require conformational switches to provide kinase activity. 


\section{Introduction}

Control of cellular growth is tightly linked to the regulation of protein synthesis through the eukaryotic initiation factor $4 \mathrm{E}$ (eIF4E). eIF4E facilitates the recruitment of mRNA to the translational machinery by binding of the mRNA 5'-cap structure (von der Haar et al., 2004) and is subject to regulation. Nutrition sensing and growth factors regulate formation of productive translation initiation complexes (Richter and Sonenberg, 2005), a process in which eIF4E activity is modulated through phosphorylation by mitogen-activated protein kinases (MAPK)-interacting kinases 1 and 2 (Mnk1 and Mnk2) (Scheper et al., 2001; Waskiewicz et al., 1997; Waskiewicz et al., 1999). Both eIF4E and Mnks bind to eIF4G within the initiation complexes. Mnks are activated in turn by MAP kinases such as extracellular signal regulated protein kinases (ERK) and p38 in response to cytokines, mitogens or cellular stress (Fukunaga and Hunter, 1997; Scheper et al., 2001; Waskiewicz et al., 1997).

The regulatory effects of Mnk-mediated phosphorylation of eIF4E on translation are still controversial (Knauf et al., 2001; Nikolcheva et al., 2002; Ueda et al., 2004) as are the biological consequences of the lack of Mnk activity in mice and fly. Mice homozygous for a targeted disruption of either one or both Mnk genes show no signs of abnormal development (Ueda et al., 2004), whereas loss of the single Drosophila Mnk homolog, Lk6, produces a dietdependent and eIF4E-mediated growth phenotype (Arquier et al., 2005; Reiling et al., 2005).

Humans possess two mnk genes, both of which encode two alternatively spliced transcripts, resulting in the proteins Mnk1a, Mnk1b, Mnk2a and Mnk2b (Figure 1A; (O'Loghlen et al., 2004; Scheper et al., 2003)). Sequence alignments reveal a central kinase domain in Mnks, which can be placed into the group of $\mathrm{Ca}^{2+} /$ calmodulin modulated protein kinases (CaMK) (Manning et al., 2002) (Figure 1B) and which is unaffected by alternative splicing (Figure 1A). We have investigated human Mnk2 as a representative of the Mnks, both structurally and functionally. We present the crystal structure of a Mnk2 fragment spanning its kinase domain at $2.1 \AA$ resolution, showing a Mnk-specific zinc-binding module and an open conformation of the activation segment (Nolen et al., 2004). In addition, Mnks contain an AspPhe-Asp (DFD) motif in place of the otherwise strictly conserved magnesium-binding AspPhe-Gly (DFG), which exhibits a conformation that obstructs ATP binding. Thus, Mnks are architecturally distinct from other protein kinases. We also present structural and functional 
data on a Mnk2 mutant containing a canonical DFG motif. Our analysis revealed novel features relevant for the regulation of activity and inhibition of Mnks and demonstrate that Mnks represent a prototype variation of CaMK group kinases.

\section{Results and Discussion}

\section{Organization and Atypical Elements of Mnk2}

The central region of Mnks harbors the kinase domain of the proteins (residues 81-368 in human Mnk2; Figure 1A, B), according to which Mnks belong to the CaMK group. The Nterminal 80 residues contain a nuclear localization signal (NLS) and a binding motif for eIF4G (Figure 1A). Residues beyond position 368 contribute to the interaction with MAP kinases (Slentz-Kesler et al., 2000) (Figure 1A). Like several other CaMK group members, Mnk's are not regulated by $\mathrm{Ca}^{2+} /$ calmodulin in agreement with the lack of a sequence element for the interaction with that protein (Slentz-Kesler et al., 2000).

Protein kinases contain conserved structural elements to bind and activate their protein substrates and the co-substrate ATP (Johnson et al., 1996; Nolen et al., 2004; Taylor and Radzio-Andzelm, 1994). In addition to those, Mnks contain atypical elements such as an Asp226-Phe227-Asp228 (DFD) motif (sequence numbering is according to human Mnk2) in place of the canonical magnesium-binding Asp-Phe-Gly (DFG) motif, and three insertions (I1I3; Figure 1B), one of which contains a cluster of four conserved cysteines (I3). We asked whether and how these features affect the structure and function of the kinase domain. While we were able to express and purify the recombinant full-length Mnk2, the protein failed to crystallize. We therefore screened for alternative expression constructs, identifying a truncated version (residue 72-385) that encompasses the kinase domain (Figure 1A) and could be produced in soluble form (see Experimental Procedues). This construct is referred to as Mnk2kinase region (Mnk2-KR) herein. Significantly, non-phosphorylated Mnk2-KR is inactive but can be activated by phosphorylation via ERK2 (Figure 2), thus recapitulating the behavior of the full-length protein (Waskiewicz et al., 1997). The non-phosphorylated Mnk2-KR could be crystallized and yielded diffraction data to $2.1 \AA$ A resolution (Table 1). 
A

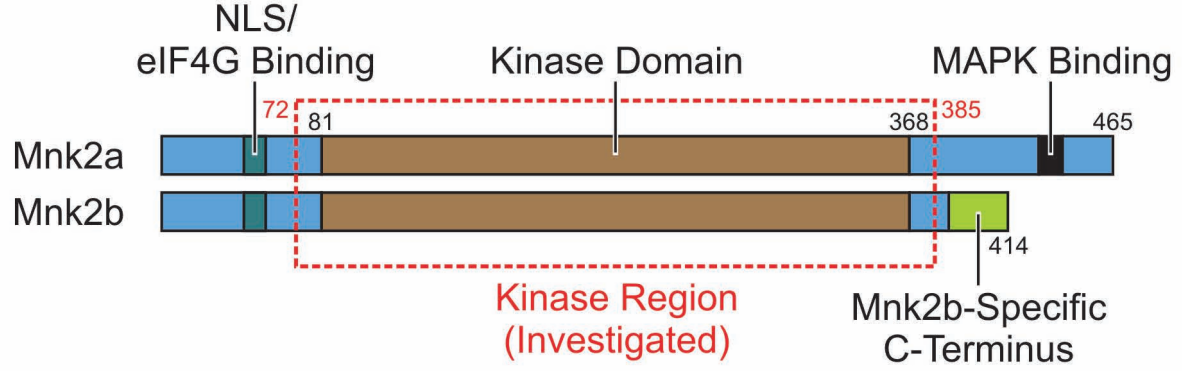

B

MNK 2

MNK 1

Lk6

R 166.5
MAPKAP

DAPK 1

Camk1a

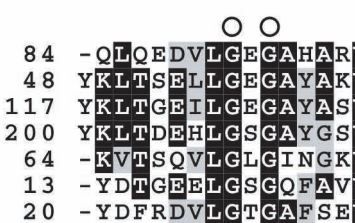

$\bigcirc$

0

O

WTINITSQEYAVKITEKQ- - - - P GHIRSRVFREVEMLYQCQG QTCVNIYTDLEYAVKVIDKI- - - - - PGHARARVFREVETFHHCQG TT TCK S I KS GVEY AVKI VDKQ G- - - - ETHSRKRI LREVNI F K TCKD VLQIFNKRTQEKFALKMLQDCKKCREKSTGLOYAAKFIKKRRTKSSRRGVSREDI EREVS I LKEIQ-

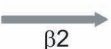

$\beta 2$ $\beta 3$

MNK2 139 HRNVLELIFFFE- - - EEDRFYLVFEKMRGGSILSHTHKR- -RHFNGLEASVVVQDVASALD MNK 1104 NKNILELIEFFE-- - DDTRFYLVFEKLQGGSILAHIQKQ- - KHFNEREASRVVRDVAAALD Lk6 173 HLGILQLIEFFE- - - DDEKFYLVFEKINGGPLLSRIQEH - - I CF SEHEASQI IKEIASGLD R166.5 257 HPNIVQLLDWFE-- - DETNFYLVMEKMRG GPLLQHILQR - - KYFTEEEARRVTKDISLALK MAPKAP 114 CPDIVRIVDVYENLYAGRKCLLIVMECLDGGELFSRIQDRGDQAFTEREASEIMKSIGEAIQ DAPK 173 HPNVITLHEVYE-- - -NKTDVILI LELVAGGELFDFLAEKE- -S LTEEEATEFLKOILNGVY CaMK1a 75 HPNIVALDDIYE----SGGHLYLIMQLVSGGELFDRIVEKG- - FYTERDASRLIFQVLDAVK

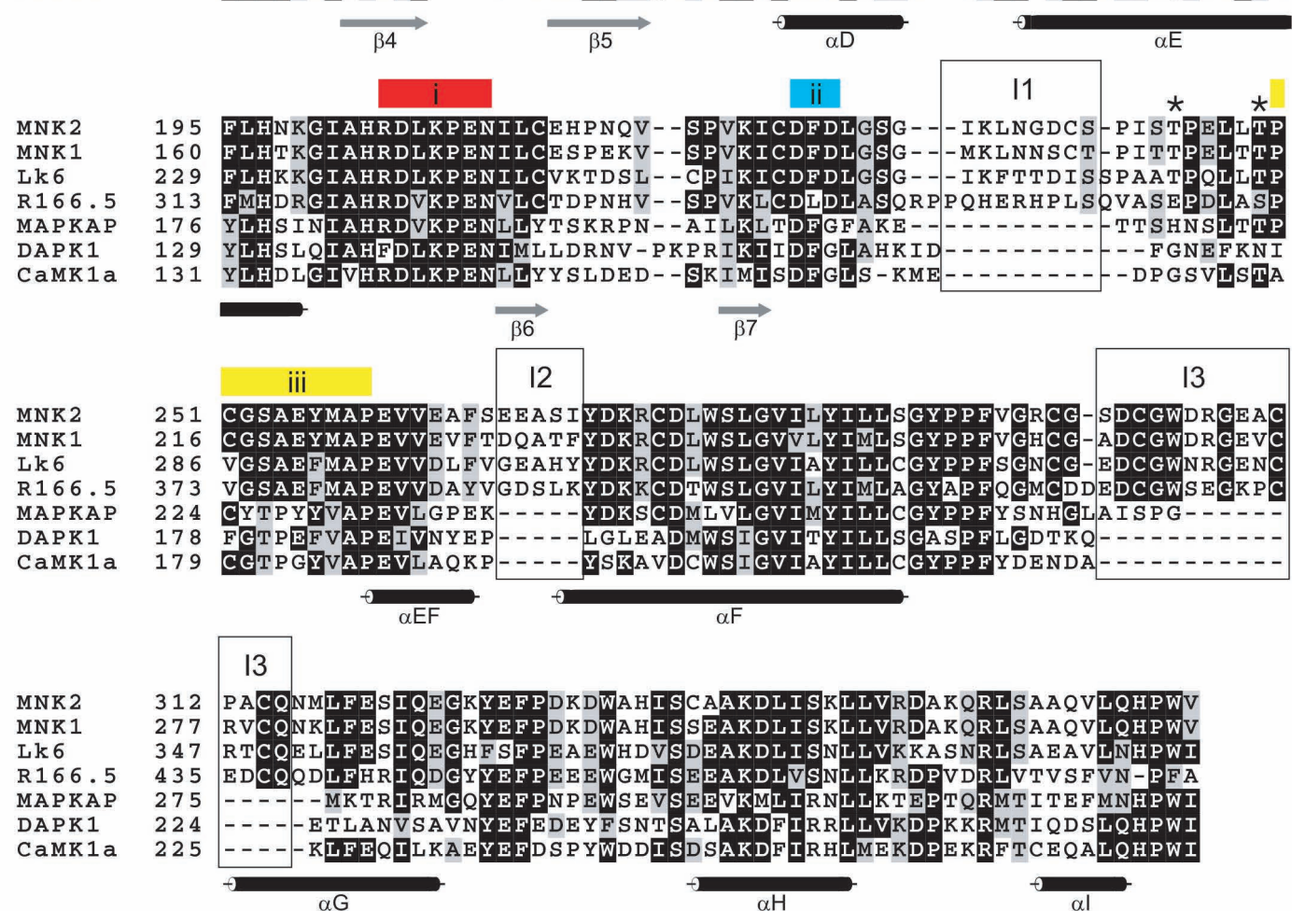

Figure 1 
Figure 1. Mnk2 Organization and Sequence Alignment

(A) Schematic comparison of the two splice variants of human Mnk2 indicating the arrangement of functional domains (as labeled). The region investigated herein (Mnk2 kinase region, Mnk2-KR) is boxed. Alternative splicing does neither affect the N-terminus nor the kinase domain. NLS - nuclear localization signal. eIF4G eukaryotic initiation factor $4 \mathrm{G}$, the scaffolding protein of the translation initiation complex which binds Mnk1 and Mnk2 (Pyronnet et al., 1999; Scheper et al., 2001).

(B) Sequence alignment of the kinase domains of human Mnk1 and Mnk2, the Drosophila and C. elegans Mnk orthologs (Lk6 and R166.5, respectively) and three human CaMK group kinases of known structure (MAPKAP MAP kinase-activated protein-kinase). Mnk2 numbering refers to a recently reported sequence (Slentz-Kesler et al., 2000). Secondary structure elements as found in Mnk2-KR are indicated below the alignment. Stars indicate phosphorylation sites (Scheper et al., 2001). The catalytic loop (i), the DFD motif (DFG in other kinases; ii) and the $\mathrm{P}+1$ loop (iii) are marked with colored bars. Insertions characteristic for Mnks are boxed (I1-I3). Open circles mark Gly91 and Gly93 of the glycine rich loop, Lys113 and Glu129 known to be important for ATP binding (Taylor and Radzio-Andzelm, 1994), filled circles mark Gly164 and Gly165 of the hinge region separating the Nterminal and C-terminal lobes.

\section{Structure Solution and Architecture of the Mnk2 Kinase Region}

The structure of Mnk2-KR was solved by molecular replacement using as a search model the structure of the death-associated protein kinase 1 (DAPK1; PDB ID 1JKS; (Tereshko et al., 2001)), another CaMK group member that exhibits about 30\% amino acid identity with Mnk2-KR. The final Mnk2-KR model and the DAPK1 structure compare with an average rmsd of $1.32 \AA$ over 211 matching $\mathrm{C} \alpha$ atoms. The Mnk2-KR crystal structure was refined to a final $\mathrm{R} / \mathrm{R}_{\text {free }}$ value of $21.5 / 25.4 \%$ maintaining good overall geometry (Table 1 ). 16 residues at the C-terminus of Mnk2-KR, which do not pertain to the core kinase domain (Figure 1A), as well as residues 232-250 (part of the activation segment) and 306-309 (part of insertion I3), which are situated in flexible regions, did not show up in the final $2 \mathrm{~F}_{\mathrm{o}}-\mathrm{F}_{\mathrm{c}}$ electron density and have been omitted from the model.

Mnk2-KR displays the typical bilobal arrangement of a protein kinase with the ATP binding cleft sandwiched between an N-terminal and a C-terminal lobe (Figure 3A). The Nterminal lobe contains a twisted $\beta$-sheet $(\beta 2-\beta 5$; numbering of secondary structure elements is according to the standard nomenclature of kinase topology; (Knighton et al., 1991a)) and a $\alpha$ helix $(\alpha \mathrm{C})$. While the N-terminal part of the protein runs in antiparallel fashion along the edge of strand $\beta 2$, it does not engage in continuous $\beta$-type backbone pairing to the $\beta$-sheet and the present structure of Mnk2-KR therefore formally lacks strand $\beta 1$ (Figures 1B, 3A). The conserved residue Glu129 of helix $\alpha \mathrm{C}$ participates in an ionic interaction with Lys113 in $\beta 3$, as required for stabilizing the ATP substrate (Taylor and Radzio-Andzelm, 1994). The glycine rich loop (residues 90-94) is flexible and contains the conserved residues Gly91 and Gly93 playing key roles in ATP binding (Taylor and Radzio-Andzelm, 1994). The C-terminal lobe 


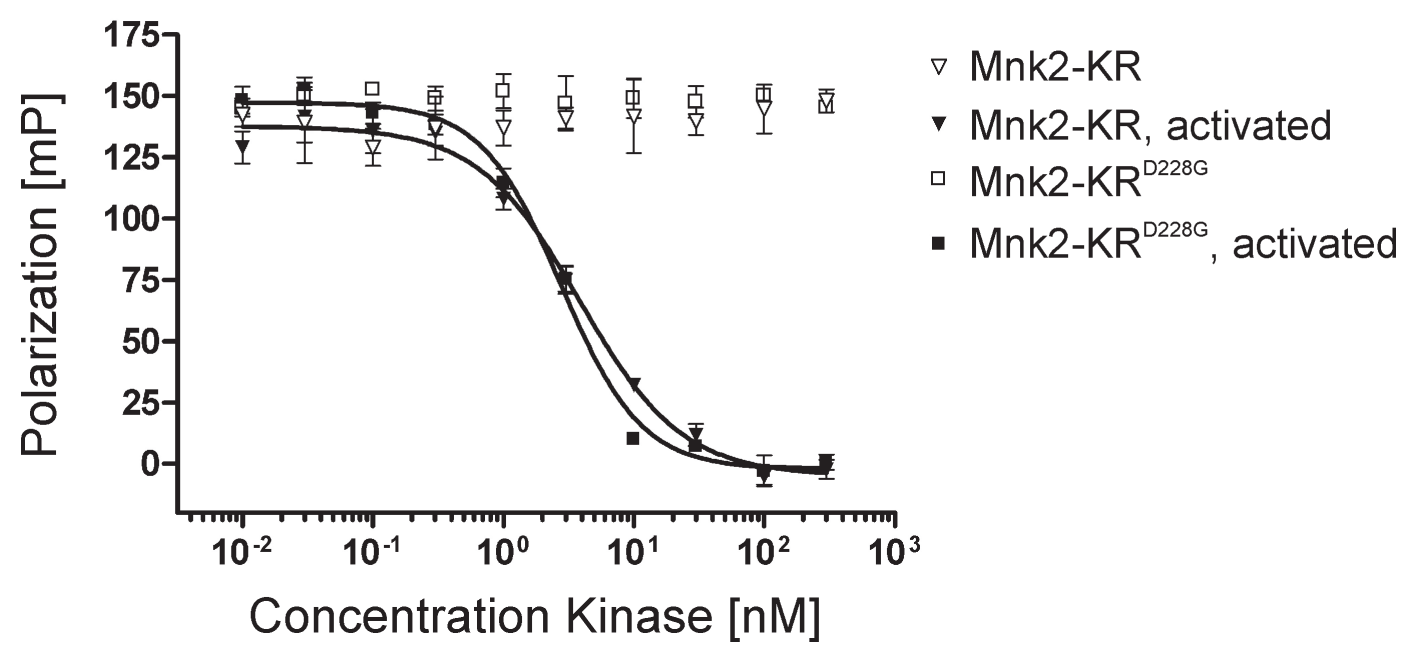

Figure 2. Catalytic Activity of Mnk2-KR and Mnk2-KR ${ }^{\mathrm{D228G}}$

Kinase activity profiles of Mnk2-KR (wild type and D228G mutant) before and after activation by phosphorylation through ERK2. Kinases were titrated in the indicated concentration range into the Mnk kinase assay (see Experimental Procedures). Data points represent means of triplicates, error bars indicate one standard deviation. The data have been subjected to non-linear regression analysis using the four parameter logistic equation (GraphPad Prism software). Results demonstrate that while activation is necessary for both wild type Mnk2-KR and the D228G mutant, the activated wild type and mutant kinase domains exhibit almost identical kinase activity (calculated $\mathrm{EC}_{50}$ s for $\mathrm{Mnk} 2-\mathrm{KR}$ and $\mathrm{Mnk} 2-\mathrm{KR}^{\mathrm{D} 228 \mathrm{G}}$ are 3.8 and $2.8 \mathrm{nM}$, respectively). 
(residues 166-368) starts downstream of the 'hinge' connector region (Gly164, Gly165) and contains a predominantly hydrophobic four-helix bundle $(\alpha \mathrm{D}-\alpha \mathrm{F}, \alpha \mathrm{H})$. Arg204, which defines the RD-kinases that are often activated by phosphorylation (Johnson et al., 1996), the catalytic aspartate (Asp205) and Asn210, which serves in active kinases to coordinates a catalytic magnesium ion (Adams, 2001), are situated in the catalytic loop (C-loop, residues 204-210). In addition, the C-terminal lobe harbors the activation segment (residues 226-260; (Nolen et al., 2004); Figure 3A), which is required for binding the protein substrate.

\section{Inhibitory Conformation of the Activation Segment}

The magnesium-binding loop (residues surrounding the DFD motif), the activation loop (disordered residues preceding the $\mathrm{P}+1$ loop) and the $\mathrm{P}+1$ loop (residues 250-259) constitute the activation segment (Figure 3A; (Nolen et al., 2004)). It is followed C-terminally by the short helix $\alpha E F$ (residues 260-265) and the long helix $\alpha F$ (residues 270-290), the latter being part of the four-helix bundle in the C-terminal lobe (Figure 3A). The $\mathrm{P}+1$ loop was shown to interact with a residue adjacent to the phosphorylation site (Knighton et al., 1991b). Together with the adjoining helix $\alpha E F$, the $\mathrm{P}+1$ loop represents subdomain VIII (Hanks and Quinn, 1991), which in active protein kinases is cradled in a predominantly hydrophobic environment provided by $\alpha \mathrm{F}, \alpha \mathrm{G}$ and the C-loop. In the present Mnk2-KR crystal structure helix $\alpha \mathrm{F}$ is $\mathrm{N}$ terminally extended due to a Mnk-specific insertion (I2; Figure 1B). As a consequence, the protein disengages the canonical intramolecular interaction between subdomain VIII and the $\alpha \mathrm{F} / \alpha \mathrm{G} / \mathrm{C}$-loop region and instead two symmetry-related, neighboring molecules in the crystal reciprocally insert their activation segments into the C-terminal lobe of the symmetry mate (Figure 3B). This intermolecular interaction observed for Mnk2-KR maintains conserved polar interactions between subdomain VIII and the $\alpha \mathrm{F} / \alpha \mathrm{G} / \mathrm{C}$-loop region, which are normally built up intramolecularly (as seen, e.g., in DAPK1; Figure 3C, Supplementary Figure 1), including a hydrogen bond between Ser253 (P+1 loop) and Asp205 (C-loop) as well as a Glu260-Arg356 ion pair (Figure 3C).

Because protein kinases require the interaction of the $\mathrm{P}+1$ loop with the $\mathrm{C}$-loop for substrate binding and since such an interaction is fostered intermolecularly in the present crystal structure, the arrangement of two Mnk2-KR molecules within the crystals suggested a functionally relevant dimer formation. However, multi-angle laser light scattering and 
analytical gel filtration chromatography showed that Mnk2-KR is a monomer in solution (data not shown). Therefore, the interaction of the $\mathrm{P}+1$ loop and the $\mathrm{C}-$ loop as required for substrate binding is not promoted by intermolecular contacts in solution. An extruded activation segment as observed in the present structure could therefore be one reason, why nonphosphorylated Mnk2 and Mnk2-KR are inactive (Figure 2). Upon phosphorylation the proteins acquire kinase activity (Figure 2), consistent with the notion that after phosphorylation the activation segment of Mnk2 flips inwards so that it can establish the necessary intramolecular contacts with the $\alpha \mathrm{F} / \alpha \mathrm{G} / \mathrm{C}$-loop region. Significantly, in the present inactive conformation Arg204 from the C-loop forms a salt bridge with Asp273 in helix $\alpha \mathrm{F}$ (Figure 3C) that immediately follows the Mnk-specific insertion I2 (Figure 1B). Thus, by positioning Asp273, Arg204 stabilizes the extruded conformation of the activation segment in inactive Mnk2-KR. As known from other RD-kinases (Johnson et al., 1996), Arg204 engages in contacts with a phosphate group after activation. The engagement of Arg204 in an alternative interaction with a phosphate after phosphorylation would release Asp273, which in turn could allow for the structural rearrangement of the activation segment required for activity.

\section{Inhibitory Conformation of the Magnesium-Binding Loop}

Protein kinases contain a conserved DFG motif (residues 226-228; Figure 3A). The first

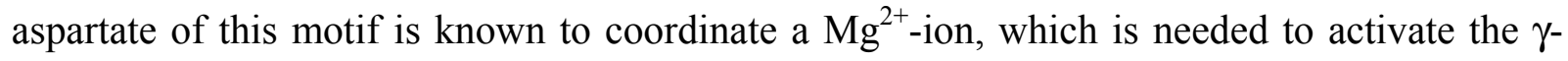
phosphate of ATP through polarization (Taylor and Radzio-Andzelm, 1994). The Mnk2-KR contains a DFD motif (residues 226-228) in place of the canonical DFG motif normally found in protein kinases. This DFD fingerprint is unique within the human kinome but conserved within the Mnk subfamily (Figure 1B). As compared to the DFG motif of, for example, DAPK1, the DFD motif of the Mnk2-KR is rotated by almost $180^{\circ}$ (Figures 4, 5A). As a result, Phe227 is displaced from its standard position in active kinases and sticks into the ATP binding pocket (Figure 4A), thus excluding ATP from its binding site. The residue also blocks an access route for the nucleotide to the pocket (Figure 4B). Thus, the arrangement of the DFD motif in Mnk2-KR interferes with the generic mode of ATP binding (Figure 4C).

The present conformation of the DFD motif is stabilized (i) by a hydrogen bonding interaction of the carbonyl oxygen of Phe227 with the side chain amino group of Lys113; and 
A
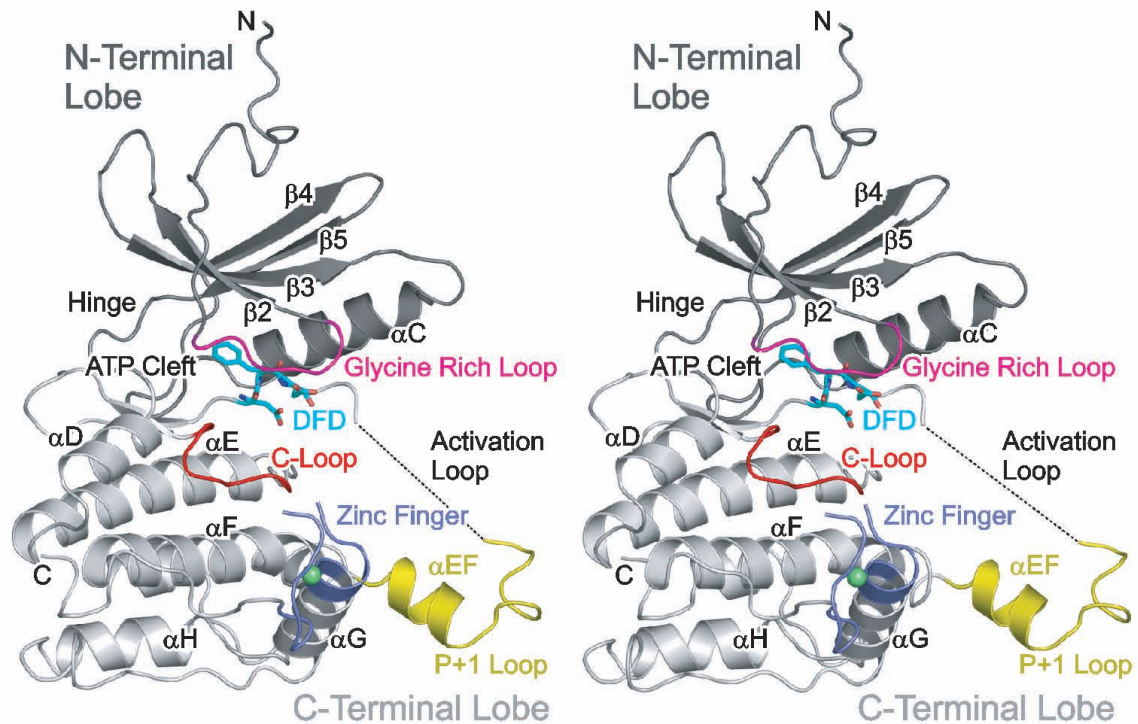

B
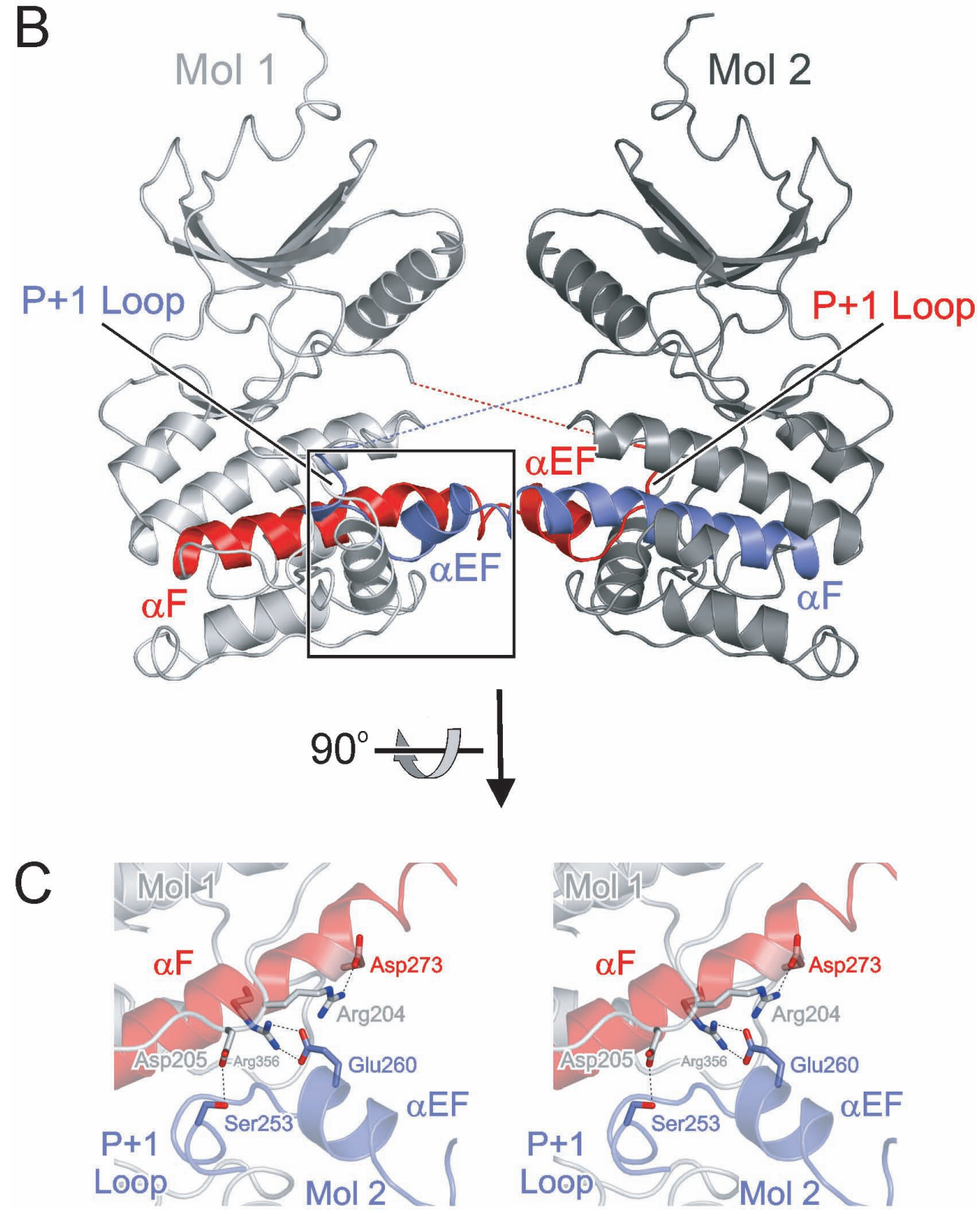

Figure 3 
Figure 3. Overall Structure of Mnk2-KR and the Extruded Activation Segment

(A) Stereo ribbon plot displaying the overall Mnk2-KR structure. Prominent structural elements are displayed in colors and labeled according to standard nomenclature (Knighton et al., 1991a). The magnesium-binding loop (around the DFD motif), the activation loop and the P+1 loop constitute the activation segment. Subdomain VIII is in yellow (Hanks and Quinn, 1991). Note that the glycine rich loop (pink) exhibits partially disordered side chains and that residues Gly232 to Cys 251 are missing in the final electron density (dotted line). The missing portion encompasses part of the activation loop including Thr244 and Thr249 that are phosphorylated by upstream activating kinases. Another region with partially missing electron density and high temperature factors corresponds to the insertion with the specific cysteine cluster (residues 304-310; tip of the zinc finger). Green sphere: zinc ion.

(B) Side view of two neighboring Mnk2-KR molecules in the crystal lattice (Mol1 and Mol2, light and dark gray, respectively), which show a reciprocal interaction via the $\mathrm{P}+1$ loop and helix $\alpha \mathrm{EF}$ (red for Mol1, blue for Mol2). The left molecule (Mol1) is rotated $20^{\circ}$ clockwise about the vertical axis compared to the view in (A).

(C) Stereo ribbon plot showing details of the interaction of the $\alpha E F / P+1$ loop portion of one molecule (Mol 2, blue) with the C-terminal lobe of a neighboring molecule in the crystal (Mol1, gray with $\alpha \mathrm{F}$ in red). Residues involved in conserved polar interaction are highlighted as sticks. Atoms are color-coded by atom type (carbon as the respective protein backbones; oxygen - red; nitrogen - blue). The view is on the boxed region in (B) rotated $90^{\circ}$ about the horizontal axis as indicated.

(ii) by positioning of the Phe 227 side chain in a hydrophobic pocket formed primarily by Leu143 and the non-conserved Phe159 (which corresponds to the so called gatekeeper residue that acts as selectivity filter for ATP-competitive drugs (Cohen et al., 2005); Figure 4A). The Mnk-specific Asp228 does not participate in stabilizing this conformation by direct interactions.

The non-canonical arrangement of the DFD motif in Mnk2-KR is referred to as the 'DFG/D-out' conformation, as opposed to the standard 'DFG/D-in' conformation as found e.g. in DAPK1 (Tereshko et al., 2001). A DFG/D-out conformation in the $\mathrm{Mg}^{2+}$-binding loop was initially found in the apo-form of the insulin receptor kinase (IRK), a tyrosine kinase (TK) (Hubbard et al., 1994). A similar DFG/D-out conformation is observed in co-crystals of tyrosine kinases such as the cellular protein encoded by Abelson gene (c-Abl), cKIT and the vascular endothelial growth factor receptor (VEGF-R) kinase with their inhibitors Gleevec and AAL-993, respectively, (Nagar et al., 2002). It is also found in the Ser/Thr kinase p38 when bound to diaryl urea scaffold molecules (Figure 4D; (Pargellis et al., 2002)). The latter substances, termed 'allosteric inhibitors', switch the DFG/D-in conformation found in the inhibitor-free p38 (Wilson et al., 1996) into a DFG/D-out conformation, in which the central phenylalanine blocks the ATP binding by sterical hindrance as observed with Mnk2-KR.

Since the DFG/D-out conformation would prevent productive ATP binding (Figure 4C), a switch of DFG/D-out into DFG/D-in is prerequisite for ATP binding in Mnk2-KR. The proposed structural rearrangement can be modeled without sterical clashes (Figure 4A). 

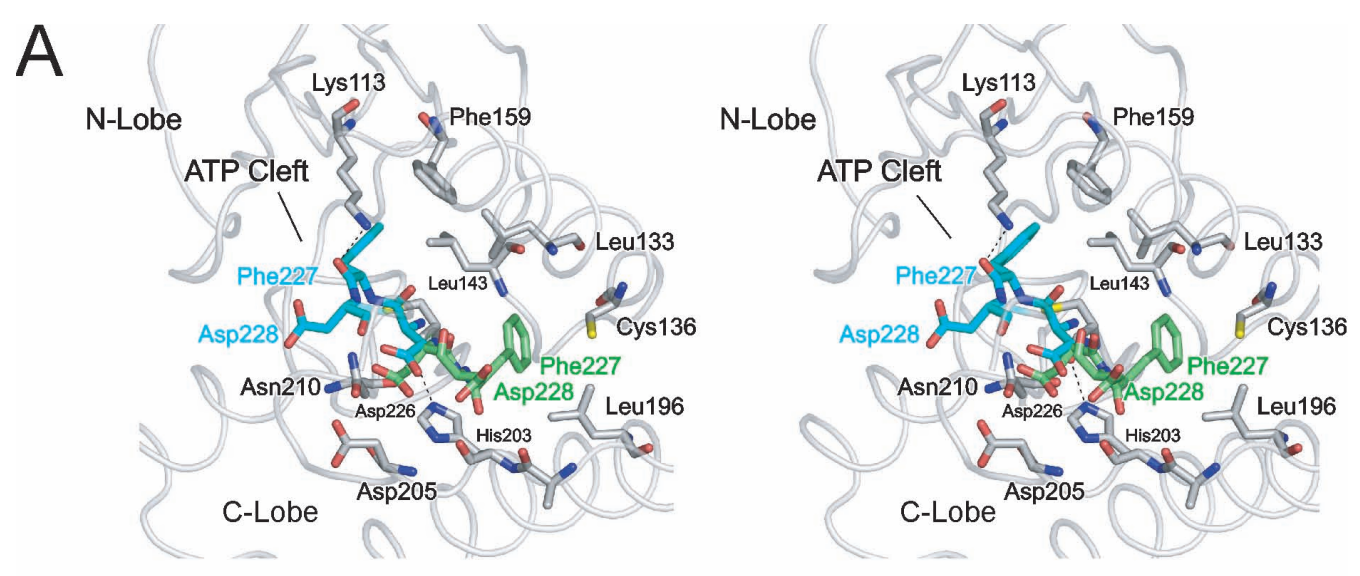

B
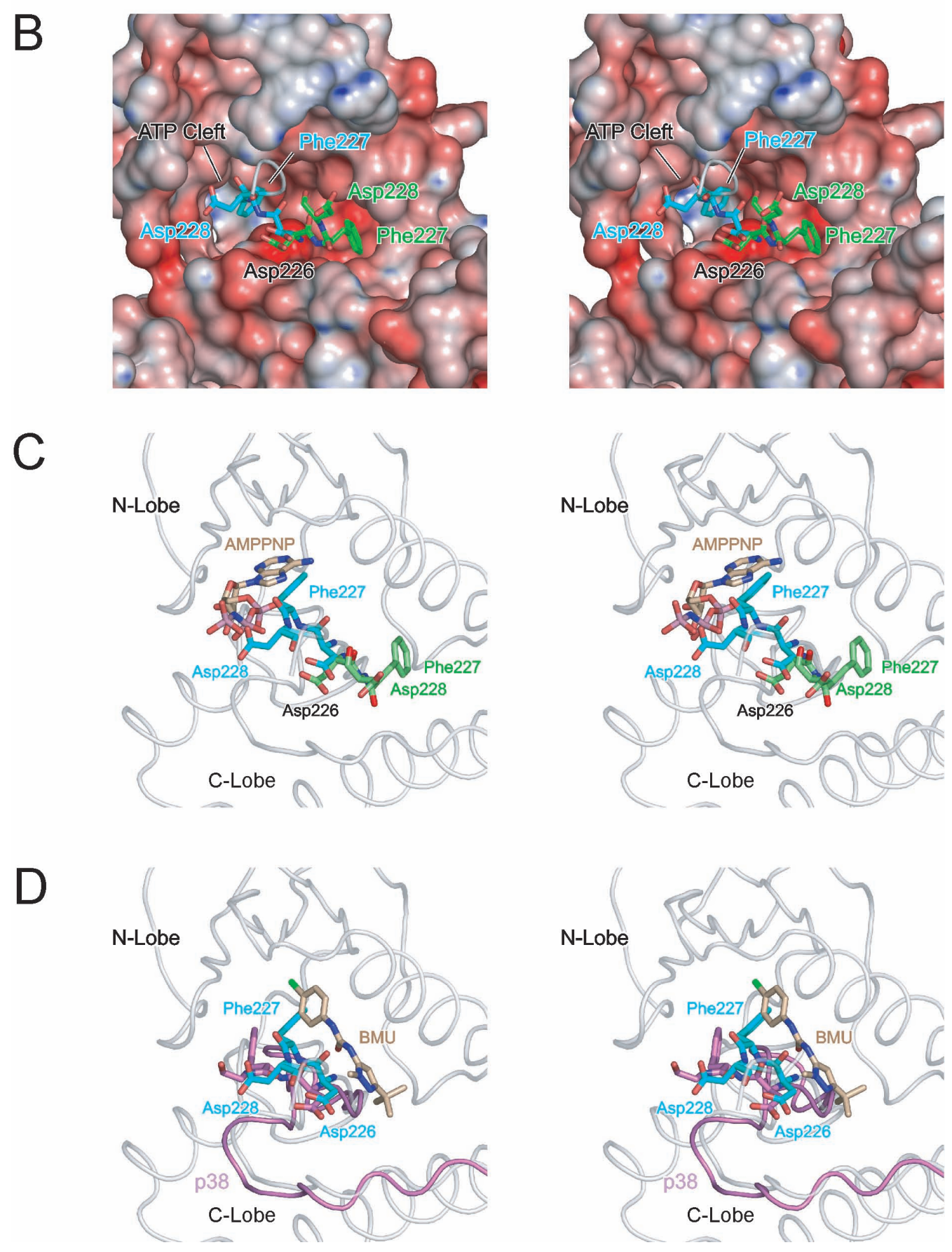

Figure 4 
Figure 4. Neighborhood of the DFD Motif

(A) Close-up stereo view of the DFD region and the ATP binding cleft. The DFG/D-out conformation of wild type Mnk2-KR is indicated by a stick representation for Asp226, Phe227 and Asp228 on the upper left with Phe227 and Asp228 poking into the ATP binding cleft (carbon - cyan). A DFG/D-in conformation (lower right; carbon - green) has been modeled according to the DFG/D-in conformation seen in other kinases and as observed for the Asp228Gly mutant of Mnk2-KR (see Figure 5B). A backbone trace of Mnk2-KR is shown as a semitransparent gray tube. Residues within a radius of $4 \AA$ around the of the DFD motif in either the DFG/D-in or -out conformations are displayed as sticks (carbon - gray). Direct interactions with the protein matrix, which stabilize the DFG/D-out conformation, are indicated by dashed lines. Phe227 comes to lie in two different hydrophobic pockets in the two different conformations. No obstacle for adoption of a DFG/D-in conformation is visible. Relative to Figure $3 \mathrm{~A}$, the molecule has been rotated $90^{\circ}$ clockwise about the vertical axis.

(B) Stereo view of the molecular surface of Mnk2-KR color-coded by electrostatic potential (blue - positive charge; red - negative charge) with the two conformations of the DFD motif as a stick representation (colorcoding as in (A)). The ATP binding cleft is pointed out. Asp228 in either conformation is well accessible to the aqueous solvent. The DFG/D-out conformation not only positions Phe227 and Asp228 in the ATP binding cleft but also obstructs access to this cleft from the front. The molecule has been rotated by $30^{\circ}$ about the horizontal axis (N-terminal lobe to back) relative to (A) in order to afford an unobstructed view into the DFD pockets.

(C) Same view as in (A) with a non-hydrolyzeable ATP analog (adenosine 5'-[ $\beta$, $\gamma$-imido]-triphosphate (AMPPNP); carbon - beige; phosphorus - violet) superimposed as seen in the co-crystal structure with DAPK1 (PDB ID 1IG1). In the DFG/D-out conformation the adenine base clashes with the side chain of Phe227, the phosphate groups clash with the side chain of Asp228.

(D) The same view as in (A) and (C) with only the DFG/D-out conformation shown. The DFG-region of a p38BMU inhibitor complex (PDB ID 1KV1) is shown for comparison (magenta tube; DFG in stick representation; carbon - magenta) as seen after global superpositioning of the protein structures. The BMU inhibitor (carbon beige; chloride - green) occupies part of the DFG/D-in binding pocket and induces a DFG/D-out conformation in p38.

\section{Replacement of DFD by the Canonical DFG Motif}

As described, we did not observe any direct interactions of the Mnk-specific Asp228 of the DFD motif with other protein residues, questioning its importance for the DFG/D-out conformation. To clarify this point, we replaced Asp228 by a glycine and solved the corresponding Mnk2-KR ${ }^{\mathrm{D} 228 \mathrm{G}}$ structure at $3.2 \AA$ resolution. The Fo-Fc 'omit' electron density map of the DFG region suggests that the Mnk2-KR ${ }^{\mathrm{D} 28 \mathrm{G}}$ molecules in the crystals adopt both the DFG/D-in and the DFG/D-out conformation (Figure 5B). Therefore, the replacement of the DFD motif in Mnks by the generic DFG motif destabilizes DFG/D-out in favor of DFG/Din. The alternative conformation observed in the $\mathrm{Mnk} 2-\mathrm{KR}^{\mathrm{D} 228 \mathrm{G}}$ mutant also shows that there are indeed no structural constrains that would prevent the switch of the DFD motif into DFG/D-in (Figure 4A).

Together, the above observations suggest that Asp228 moderately stabilizes DFG/D-out although it is not involved in direct intramolecular interactions in the present crystal structure. When modeling a DFD/G-in conformation of a DFD motif based on the structure of the Mnk2-KR ${ }^{\mathrm{D} 28 \mathrm{G}}$ mutant (Figure 4A) no direct interactions involving Asp228 can be discerned either. The overall exposure of Asp228 to the aqueous environment in either conformation 
A

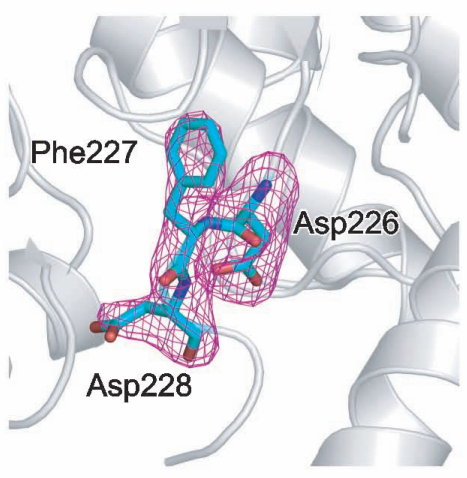

B

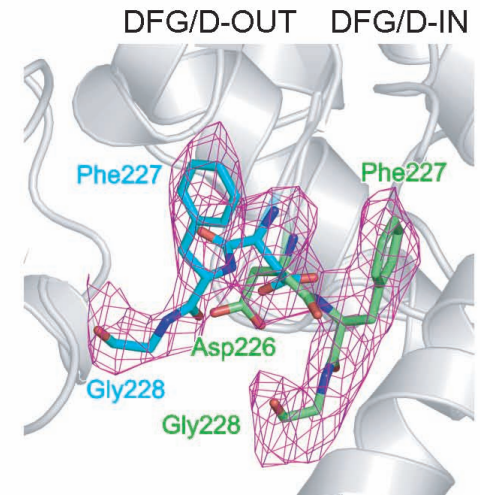

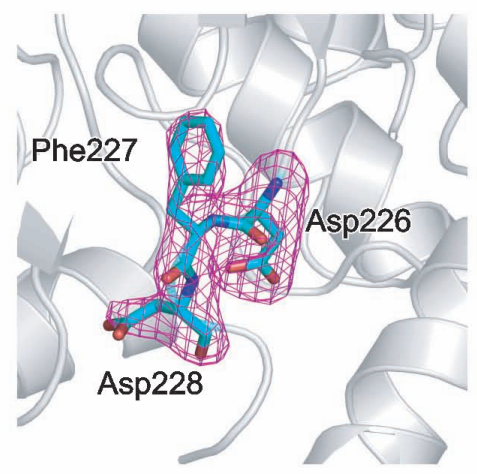

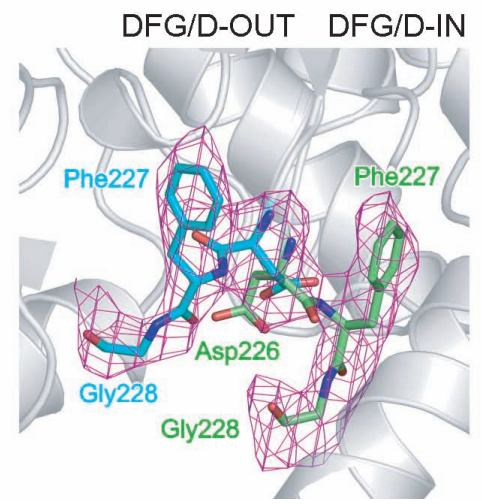

I II III

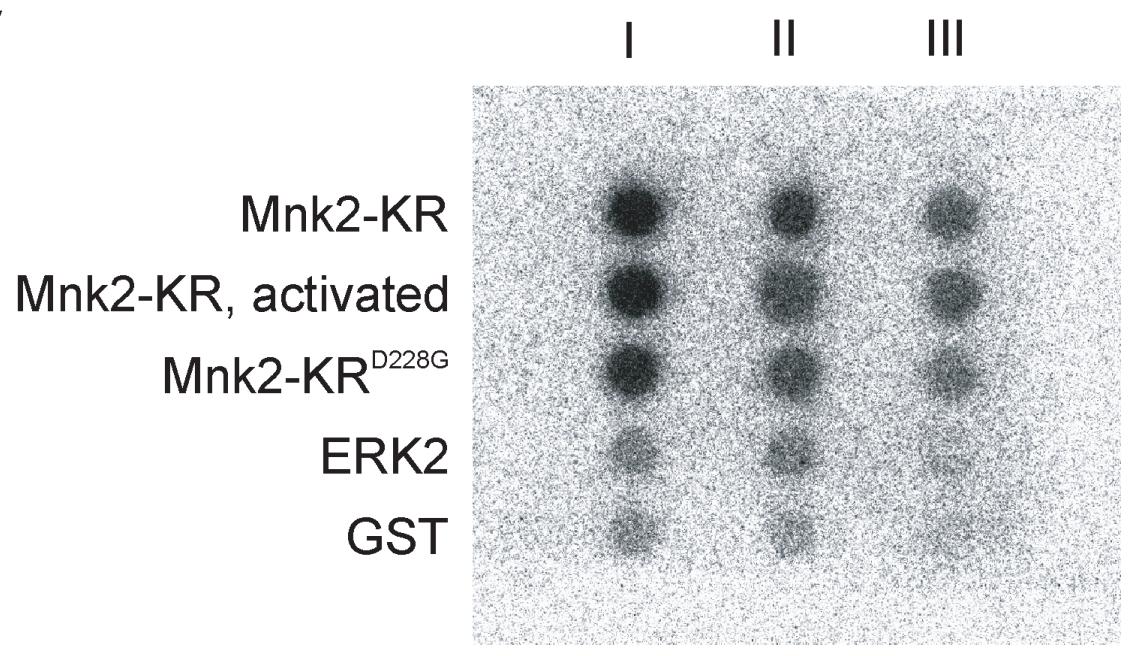

Figure 5. Conformation of the DFD Motif and ATP Binding

(A) Stereo plot showing the Fo-Fc 'omit' electron density contoured at the $3 \sigma$ level covering the DFD motif (sticks; carbon - cyan) in wild type Mnk2-KR (gray ribbon). Relative to Figure 3A, the molecule has been rotated $90^{\circ}$ clockwise about the vertical axis and $90^{\circ}$ about the horizontal axis (N-terminal lobe to front), affording a view from the N-terminal lobe onto the motif. Only the DFG/D-out conformation is visible in wild type Mnk2KR.

(B) Stereo plot showing the Fo-Fc 'omit' electron density contoured at the $2.5 \sigma$ level covering the DFG motif in Mnk2-KR ${ }^{\mathrm{D} 228 \mathrm{G}}$. The DFG motif is found in both a DFD/G-in (carbon green) and in a DFD/G-out (carbon cyan) conformation in the mutant.

(C) Dot blot assaying the binding of $\alpha^{32}$-ATP to the indicated proteins at concentrations of $2.8 \mu \mathrm{M}$ (I), $1.4 \mu \mathrm{M}$ (II) and $0.7 \mu \mathrm{M}$ (III). GST - negative control using glutathione S-transferase. 
seems to be similar as well (Figure 4B). In addition, we cannot discern decisive differences in the electrostatic potentials around the DFD motif that would favor the DFG/D-out or -in conformation (Figure 4B). One possibility is that interactions involving Asp228 ensue in a slightly modified conformation in solution and that the DFG/D-out conformation has been trapped in the crystals. Gly228 in place of Asp228 in the Mnk2-KR ${ }^{\mathrm{D} 228 \mathrm{G}}$ mutant may foster increased backbone flexibility, which could then allow the protein to adopt both conformations.

We next asked whether the DFG/D-out to DFG/D-in switch that is necessary to allow ATP binding requires factors other than ATP itself. ATP binding assays with nonphosphorylated Mnk2-KR, phosphorylated and thereby activated Mnk2-KR and with Mnk2$\mathrm{KR}^{\mathrm{D} 228 \mathrm{G}}$ showed that both Mnk2-KR and $\mathrm{Mnk} 2-\mathrm{KR}^{\mathrm{D} 228 \mathrm{G}}$ were able to bind ATP and that binding occurred irrespective of the phosphorylation-dependent activation of the proteins (Figure 5C). However, only the phosphorylated forms of Mnk2-KR and Mnk2-KR ${ }^{\mathrm{D} 228 \mathrm{G}}$ exerted kinase activity (Figure 2). These results establish that phosphorylation of Mnk2-KR is a prerequisite for catalytic activity but not for ATP binding, and that neither aspect depends on Asp228 of the DFD motif.

Since our solution studies showed that ATP is able to bind to non-phosphorylated Mnk2KR (Figure 5C), we attempted to co-crystallize the protein with ATP, ATP/Mg ${ }^{2+}$ or the nonhydrolyzeable analog AMPPNP with and without $\mathrm{Mg}^{2+}$ or to soak preformed crystals with these substances in order to directly visualize conformational changes taking place upon nucleotide binding. Despite extensive efforts, we were not able to obtain crystals of the ATPbound form. Indirectly, these negative results suggest that indeed conformational changes take place upon ATP binding.

\section{A Zinc-Binding Motif in Mnk2}

Among the Mnk-specific insertions, a region containing four conserved cysteine residues stands out (I3; Figure 1B). In the present crystal structure, the four cysteines are arranged in a manner suggesting a metal ion binding site (Figure 6A). In order to verify the presence of a metal ion in the protein, we recorded the X-ray fluorescence spectrum of frozen Mnk2-KR crystals. $\mathrm{K} \alpha$ and $\mathrm{K} \beta$ emission lines characteristic of a zinc ion were observed (Figure 6B). Significantly, $\mathrm{Zn}^{2+}$ was never added during the expression, purification or crystallization of the protein and, therefore, must have been scavenged spontaneously by the 
protein from the cellular environment. To locate the zinc ion within the Mnk2-KR structure, we reprocessed the original Mnk2-KR diffraction data keeping the Friedel pairs separated. While the diffraction data were collected at a wavelength of $1.05 \AA$, i.e. remote from the Zn Kedge (about $1.28 \AA$ ), the anomalous signal expected at that wavelength $(\sim 2.7 \mathrm{e})$ is still strong compared to the maximum signal around the edge $(\sim 3.9 \mathrm{e})$. Indeed, the Fourier map calculated from the model phases and the anomalous intensity differences revealed a single peak at $\sim 6 \sigma$ above the background, which maps to the center of the four cysteine SH-groups (Figure 6A). Therefore we conclude that Mnk2 bears a zinc finger-like module within the kinase domain.

We do not expect a direct effect of the zinc finger on the catalytic activity since it is positioned far away from the catalytic center. In zinc fingers of other proteins, zinc ions contribute to the shaping and compaction of short structural motifs, which in the following often provide a binding platform for proteins and/or nucleic acids (Krishna et al., 2003). Thus, we assume that the Mnk-specific zinc finger serves as a docking site for phosphorylation targets and/or regulators. Studies to test the significance of the Mnk specific zinc finger in vivo are in progress.

\section{Conclusions}

Herein, we have investigated a truncated form of human Mnk2 (Mnk2-KR) that covers the kinase domain and exhibits phosphorylation-dependent activity indistinguishable from the full-length protein. Our results provide evidence that Mnks represent a distinct protein kinase subfamily. This subfamily is characterized by three insertions which lead to an open conformation of the activation segment in the non-phosphorylated Mnk2-KR and a zinccoordinating cysteine cluster. Adoption of the active conformation of the activation segment after phosphorylation may be triggered by a phosphate-mediated disruption of an Arg204Asp273 salt bridge that otherwise stabilizes the inactive, extruded conformation of the activation segment. In addition, we observed a DFD motif that favors an inhibitory DFG/D-out conformation in contrast to other unliganded Ser/Thr kinases. Interestingly, the DFG/D-out conformation has no qualitative effect either on the ATP binding properties of nonphosphorylated Mnk2-KR or on the catalytic activity after phosphorylation, suggesting that it is marginally stable in the present structure. The latter notion is in agreement with the lack of direct interactions of the Mnk specific Asp228 that would fasten the DFG/D-out conformation. Since both, ATP binding and kinase activity, require an open ATP binding pocket, the switch 


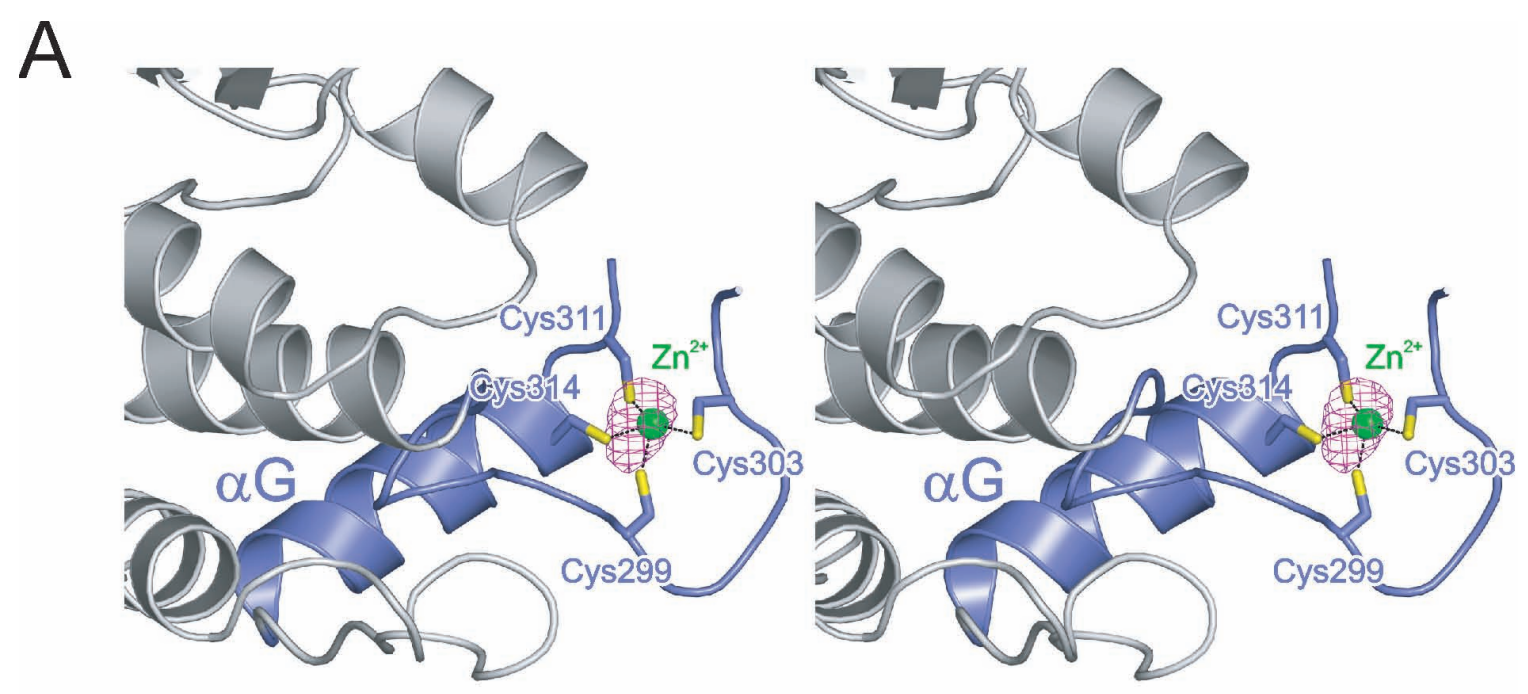

B

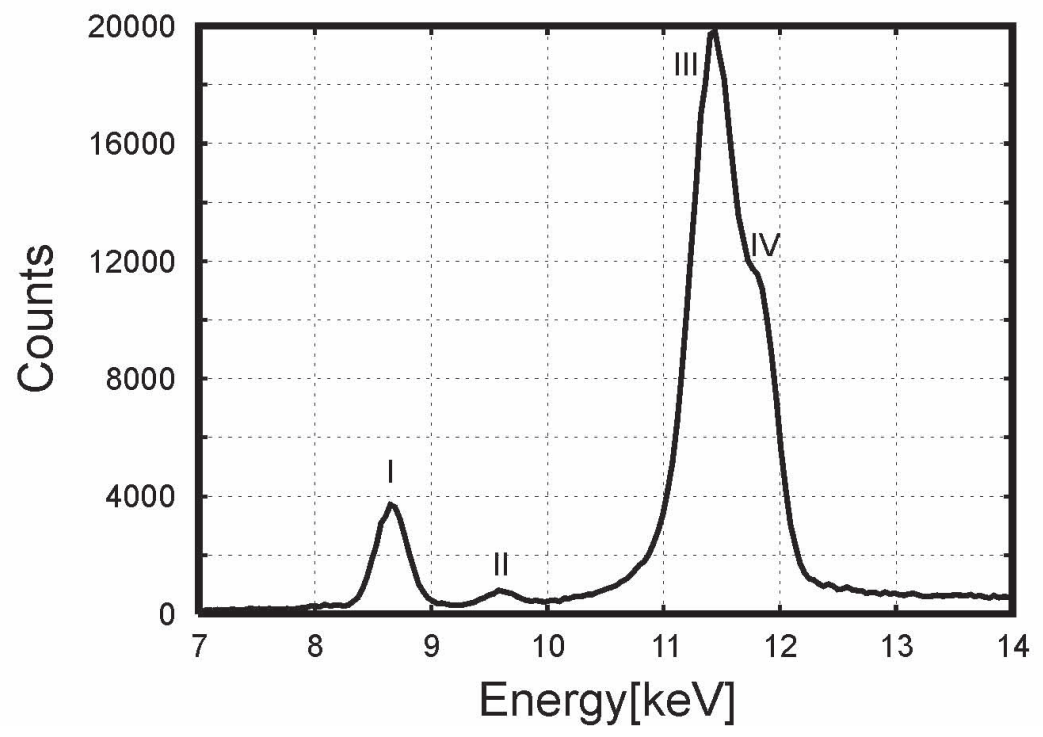

Figure 6. Zinc-Binding Site

(A) Stereo ribbon plot of the zinc finger motif in Mnk2-KR (blue). The cluster of four cysteines is indicated by sticks at the tip of the finger (carbon - blue; sulfur - yellow). They surround a zinc ion (green sphere). A Fourier map using the anomalous intensity differences and phases from the refined model is shown contoured at the $5 \sigma$ level and identifies the position of the anomalous scatterer. The region from Trp305 to Glu309 lacks unambiguous backbone density and has been omitted from the model. The molecule has been rotated $90^{\circ}$ counterclockwise about the vertical axis compared to Figure 3A.

(B) X-ray emission spectrum of native Mnk2-KR crystals irradiated with X-rays of $1.05 \AA$. Peaks in the spectrum correspond to $\mathrm{I}=\mathrm{Zn}-\mathrm{K} \alpha$ line, $\mathrm{II}=\mathrm{Zn}-\mathrm{K} \beta$ line, III Compton scattering, IV elastic scattering. 
into the DFG/D-in conformation might be imposed by ATP binding per se, again consistent with a weak intramolecular stabilization of the DFG/D-out conformation in the Mnk2-KR structure. The question remains whether a regulatory potential via interconversion of DFG/Din and-OUT conformations could be realized in vivo, e.g. by interaction with other regulatory proteins or by the protein adopting other conformations in solution.

Since the DFG/D-out conformation of Mnk2 is rarely observed in inhibitor-free protein kinases, it provides a handle for specific inhibitor design that would extend the range of generic ATP-competitive compounds (Mol et al., 2004), a speculation that is supported by the binding of the DFG/D-out-inducing p38-inhibitor BIRB-796 to Mnk2 (Fabian et al., 2005). Also, the Mnk-specific Asp228 may aid inhibitor design because it renders the $\mathrm{Mg}^{2+}$-binding loop unique among Ser/Thr kinases. 


\section{Tables}

Table 1. Crystallographic Data

\begin{tabular}{|c|c|c|}
\hline & Mnk2-KR Wild Type & Mnk2-KR ${ }^{\text {D228G }}$ \\
\hline \multicolumn{3}{|l|}{ Data collection } \\
\hline Wavelength $(\AA)$ & 1.05 & 1.05 \\
\hline Space Group & $\mathrm{P}_{2} 21$ & $\mathrm{P}_{2} 21$ \\
\hline \multicolumn{3}{|l|}{ Cell Dimensions $(\AA)$} \\
\hline $\mathrm{a}(=\mathrm{b})$ & 104.5 & 104.6 \\
\hline $\mathrm{c}$ & 72.3 & 73.4 \\
\hline Resolution $(\AA)$ & $30.0-2.1(2.2-2.1)^{\mathrm{a}}$ & $30-3.2(3.3-3.2)$ \\
\hline $\mathrm{R}_{\mathrm{sym}}{ }^{\mathrm{b}}(\%)$ & $5.2(37.3)$ & $4.1(46.2)$ \\
\hline $\mathrm{I} / \sigma \mathrm{I}$ & $31.6(3.4)$ & $14.5(1.8)$ \\
\hline Completeness (\%) & $99.5(99.9)$ & $98.2(99.0)$ \\
\hline \multicolumn{3}{|l|}{ Refinement } \\
\hline Resolution $(\AA)$ & $15.0-2.1$ & $30-3.2$ \\
\hline No. Reflections & 24664 & 7768 \\
\hline $\mathrm{R}_{\text {work }} / \mathrm{R}_{\text {free }}{ }^{\mathrm{c}}(\%)$ & $21.5 / 25.4$ & $23.8 / 30.6$ \\
\hline \multicolumn{3}{|l|}{ No. Atoms } \\
\hline Protein & 2217 & 2205 \\
\hline $\mathrm{Zn}^{2+}$ & 1 & 1 \\
\hline Water & 161 & 18 \\
\hline \multicolumn{3}{|l|}{ B-factors } \\
\hline Protein & 75.8 & 75.6 \\
\hline Water & 77.4 & 53.1 \\
\hline Wilson & 60.1 & 68.5 \\
\hline \multicolumn{3}{|l|}{ Rms Deviations from Ideal } \\
\hline Bond lengths $(\AA)$ & 0.009 & 0.009 \\
\hline Bond angles $\left({ }^{\circ}\right)$ & 1.21 & 1.56 \\
\hline
\end{tabular}

a Values for the highest $0.1 \AA$ resolution shell in parentheses.

${ }^{\mathrm{b}} \mathrm{R}_{\mathrm{sym}}(\mathrm{I})=\left(\Sigma_{\mathrm{hkl}} \Sigma_{\mathrm{i}}\left[\left|I_{\mathrm{i}}(\mathrm{hkl})-<I(\mathrm{hkl})>\right|\right] / \Sigma_{\mathrm{hkl}} \Sigma_{\mathrm{i}}\left[I_{\mathrm{i}}(\mathrm{hkl})\right] ; I_{\mathrm{i}}(\mathrm{hkl})-\right.$ intensity of the $\mathrm{i}^{\text {th }}$ measurement of hkl; $<I(\mathrm{hkl})>$ - average value of hkl for all i measurements.

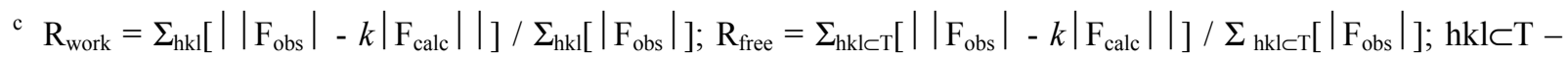
test set. 

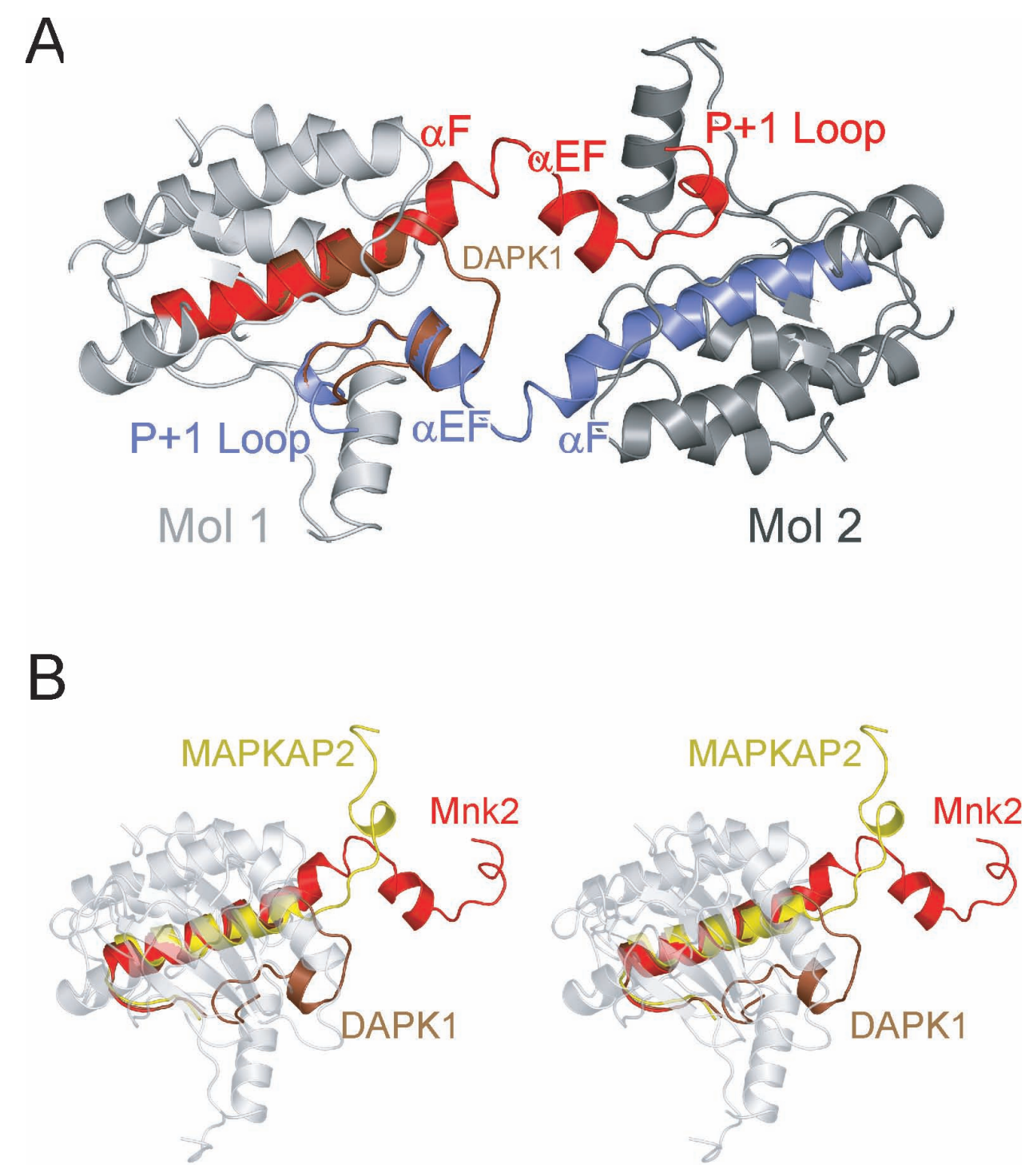

\section{Supplementary Figure 1. Open Conformation of the Activation Segment}

(A) View from the N-terminal lobe onto the arrangement of the two molecules (rotated $90^{\circ}$ about the horizontal axis compared to the view in Figure 3B). The N-terminal lobe and the upper part of the C-terminal lobe have been cut away to allow an unobstructed view onto the interaction regions. The P+1 loop region of DAPK1 is cradled intramolecularly within the body of the C-terminal lobe and is shown for comparison (brown ribbon) after global superpositioning of the DAPK1 structure (PDB ID 1JKS) onto Mol1.

(B) Stereo ribbon plot comparing the conformations of the $\mathrm{P}+1$ loop $/ \alpha E F / \alpha F$ regions in Mnk2-KR (red), MAP kinase-activated protein-kinase-2 (MAPKAP2; yellow; PDB ID 1NXK) and DAPK1 (brown; PDB ID 1JKS). The segments are shown after global superpositioning of the protein structures. The remainder of a Mnk2-KR molecule is indicated by a semi-transparent gray ribbon. The view is the same as in (A). 


\section{Experimental Procedures}

\section{Cloning}

A cDNA fragment of human Mnk2 (amino acid residues 72-385; Entrez AAG26336), which encompasses the kinase domain, was amplified using the forward/reverse primer pair 5'CGGGATCCACCGACAGCTTCTCGGGCAGG/5'ACGCGTCGACACTACCTCTGCAG GACCATGGGAG (restriction sites underlined) and cloned into the BamHI and SalI sites of the vector pGEX-4T1 (Amersham, Uppsala, Sweden). The amino acid substitution Asp228Gly was introduced into the GST-Mnk2-KR construct employing the Stratagene (Heidelberg, Germany) Quik Change Site Directed Mutagenesis kit according to the manufacturers' instructions. Mutagenesis oligonucleotides were 5'GAAGATCTGTGACTTCGGCCTGGGCAGCGGCATCAAACTC and 5'GAGTTTGATG CCGCTGCCCAGGCCGAAGTCACAGATCTTC.

\section{Protein Preparation}

Expression of GST-Mnk2-KR or GST-Mnk2-KR ${ }^{\mathrm{D} 228 \mathrm{G}}$ was performed in E. coli BL21. Cells were grown in LB medium (Merck, Darmstadt, Germany) supplemented with ampicillin $\left(100 \mu \mathrm{g} / \mathrm{ml} ; 37^{\circ} \mathrm{C}\right)$. Expression was induced at $\mathrm{OD}_{600}$ of 0.8 with $1 \mathrm{mM}$ isopropyl thiogalactoside $\left(4 \mathrm{~h} ; 25^{\circ} \mathrm{C}\right)$. Cells were harvested by centrifugation and resuspended in $10 \mathrm{ml}$ lysis buffer (50 mM Tris/HCl, pH 7.5, $200 \mathrm{mM} \mathrm{NaCl,} 5 \mathrm{mM}$ DTT) per gram wet weight. Lysates were prepared by sonication and subsequent clearing in a Sorvall SS34 rotor (18000 rpm, $45 \mathrm{~min}, 4^{\circ} \mathrm{C}$ ). The supernatant was applied to two GSTPrep FF 16/10 columns (Amersham) connected in series and equilibrated with lysis buffer.

Wild type or mutated Mnk2-KR were eluted by on-column thrombin cleavage from the GST tag. 1000 units of thrombin (Amersham) were dissolved in $60 \mathrm{ml}$ wash buffer and cycled over the columns (over night, $8^{\circ} \mathrm{C}$ ). After collection, the thrombin eluate was diluted $(1: 5$ in 50 $\mathrm{mM}$ Tris/HCl, $\mathrm{pH} 8.0$ ), applied to a $25 \mathrm{ml} \mathrm{Q}$ sepharose HP column (Amersham) and eluted with a linear gradient of sodium chloride $(50 \mathrm{mM}$ Tris/ $\mathrm{HCl}, \mathrm{pH} 8.0,0-1 \mathrm{M} \mathrm{NaCl})$. Fractions

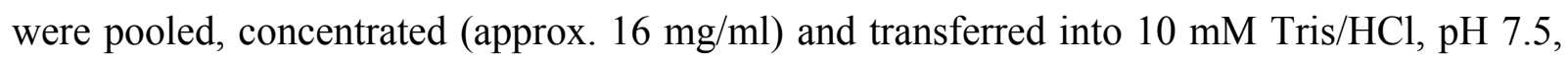
$50 \mathrm{mM} \mathrm{NaCl}, 1 \mathrm{mM}$ DTT via a PD10 column (Amersham). Typically the final protein concentration was approximately $12 \mathrm{mg} / \mathrm{ml}$. 


\section{Crystallographic Analysis}

Crystals of wild type Mnk2-KR and of Mnk2-KR ${ }^{\mathrm{D} 228 \mathrm{G}}$ were grown by vapor diffusion with a reservoir solution containing $23 \%(\mathrm{w} / \mathrm{v})$ polyacrylic acid 5100, $2 \%$ (v/v) 2-methyl-2,4pentane-diol and 0.1 M HEPES/NaOH, pH 7.7 and were frozen (liquid nitrogen) in reservoir solution supplemented with $12.5 \%$ glycerol and $12.5 \%$ ethyleneglycol. Diffraction data (Table 1) were collected on beamline BW6 (DESY, Hamburg, Germany) at 100K on a MarResearch (Norderstedt, Germany) CCD detector and processed with the HKL package (Otwinowski and Minor, 1997).

The Mnk2-KR structure was solved by molecular replacement (MolRep; (Collaborative Computational Project, 1994) using the structure coordinates of DAPK1 (PDB ID 1JKS) as the search model. Model building was conducted automatically using arp/warp (Cohen et al., 2004) and manually using Xfit (McRee, 1999). Refinement was performed with CNS (Brunger et al., 1998) and Refmac5 (Collaborative Computational Project, 1994) using standard protocols. All data between 15.0 and $2.1 \AA$ resolution were employed in the refinement, setting aside $5 \%$ of the reflections to monitor the free R-factor (Table 1 ). In the last refinement cycles, different overall anisotropic temperature factor corrections were applied to the N-terminal lobe and the C-terminal lobe using the TLS refinement option of Refmac5. The structure of Mnk2-KR ${ }^{\mathrm{D} 228 \mathrm{G}}$ was subsequently solved by molecular replacement using the refined Mnk2-KR structure and refined with CNS.

\section{Gel Filtration and Light Scattering}

Gel filtration chromatography was carried out with a SMART system using a Superdex 75 PC 3.2/30 column (Amersham). Experiments were performed at room temperature in 20 $\mathrm{mM}$ Tris/ $\mathrm{HCl}, \mathrm{pH} 7.5,100 \mathrm{mM} \mathrm{NaCl}, 1 \mathrm{mM}$ DTT at a flow rate of $0.04 \mathrm{ml} / \mathrm{min}$. The molecular weight of Mnk2-KR was estimated using standard proteins (BioRad, Munich, Germany). Multi-angle laser light scattering with a $30 \mu \mathrm{M}$ solution of Mnk2-KR in buffer A was performed as described elsewhere (Jauch et al., 2003).

\section{Kinase and Dot Blot Assays}

Mnk2, Mnk2-KR and Mnk2-KR ${ }^{\mathrm{D} 228 \mathrm{G}}(2.5 \mu \mathrm{M})$ were activated using $140 \mathrm{nM}$ preactivated ERK2 and $50 \mu \mathrm{M}$ ATP in $20 \mathrm{mM}$ HEPES/KOH, pH 7.4, $10 \mathrm{mM} \mathrm{MgCl} 2,0.25 \mathrm{mM}$ DTT and $0.05 \%$ (w/v) polyoxyethylene 20 stearylether (Brij 78; Sigma, Munich, Germany) 
for $45 \mathrm{~min}$ at $30^{\circ} \mathrm{C}$. In vitro kinase activity was assayed in the presence of $30 \mu \mathrm{M}$ eIF4Ederived substrate peptide and $20 \mu \mathrm{M}$ ATP in a buffer of $20 \mathrm{mM}$ HEPES/KOH, pH 7.4, $10 \mathrm{mM}$ $\mathrm{MgCl}_{2}, 0.5 \mathrm{mM}$ DTT, $0.1 \%(\mathrm{w} / \mathrm{v})$ bovine serum albumin and $0.01 \%(\mathrm{w} / \mathrm{v})$ Pluronic F127 (Sigma). Reactions $\left(40 \mathrm{~min}, 30^{\circ} \mathrm{C}\right)$ were terminated by EDTA addition. Substrate phosphorylation was detected based on a competitive fluorescence polarization format (Seethala and Menzel, 1998).

For the dot blots, $0.7-5 \mu \mathrm{M}$ protein were incubated with a $10 \mu \mathrm{M}$ mixture of ATP and $\alpha^{32}$ P-ATP $(0.2 \mu \mathrm{Ci}$; Hartmann Analytic, Braunschweig, Germany) in $10 \mathrm{mM}$ Tris/HCl, $\mathrm{pH}$ 7.5, $100 \mathrm{mM} \mathrm{NaCl}, 10 \mathrm{mM} \mathrm{MgCl} 2$ (30-60 minutes at room temperature). Proteins were transferred onto nitrocellulose membranes using the Minifold I vacuum filtration system (Schleicher\&Schüll, Dassell, Germany), washed and detected by autoradiography.

\section{Accession Numbers}

Coordinates and structure factors have been submitted to the RSCB Protein Databank (http://www.rcsb.org/pdb/) with accession codes $2 \mathrm{AC} 3$ and $2 \mathrm{AC} 5$ and will be released upon publication.

\section{Acknowledgments}

We thank Monique Erling for excellent technical support in protein production and purification and Gleb Bourenkov (DESY, Hamburg) for help with diffraction studies. Work was in part supported by the Max-Planck Gesellschaft (HJ and MCW). $\mathrm{CN}$ is a fellow of the Boehringer Ingelheim Fonds. This work is part of the scientific research conducted in the International Ph.D. Program Molecular Biology - International Max Planck Research School at the Georg August University, Göttingen, Germany. 


\section{References}

Adams, J. A. (2001). Kinetic and catalytic mechanisms of protein kinases. Chem Rev 101, 2271-2290.

Arquier, N., Bourouis, M., Colombani, J., and Leopold, P. (2005). Drosophila Lk6 kinase controls phosphorylation of eukaryotic translation initiation factor $4 \mathrm{E}$ and promotes normal growth and development. Curr Biol 15, 19-23.

Brunger, A. T., Adams, P. D., Clore, G. M., DeLano, W. L., Gros, P., Grosse-Kunstleve, R. W., Jiang, J. S., Kuszewski, J., Nilges, M., Pannu, N. S., et al. (1998). Crystallography \& NMR system: A new software suite for macromolecular structure determination. Acta Crystallogr. D 54, 905-921.

Cohen, M. S., Zhang, C., Shokat, K. M., and Taunton, J. (2005). Structural bioinformaticsbased design of selective, irreversible kinase inhibitors. Science 308, 1318-1321.

Cohen, S. X., Morris, R. J., Fernandez, F. J., Ben Jelloul, M., Kakaris, M., Parthasarathy, V., Lamzin, V. S., Kleywegt, G. J., and Perrakis, A. (2004). Towards complete validated models in the next generation of ARP/wARP. Acta Crystallogr. D 60, 2222-2229.

Collaborative Computational Project, N. (1994). The CCP4 Suite: Programs for protein crystallography. Acta Crystallogr. D 50, 760-763.

Fabian, M. A., Biggs, W. H., Treiber, D. K., Atteridge, C. E., Azimioara, M. D., Benedetti, M. G., Carter, T. A., Ciceri, P., Edeen, P. T., Floyd, M., et al. (2005). A small moleculekinase interaction map for clinical kinase inhibitors. Nat Biotechnol. 23, 329-36

Fukunaga, R., and Hunter, T. (1997). MNK1, a new MAP kinase-activated protein kinase, isolated by a novel expression screening method for identifying protein kinase substrates. Embo J 16, 1921-1933.

Hanks, S. K., and Quinn, A. M. (1991). Protein kinase catalytic domain sequence database: identification of conserved features of primary structure and classification of family members. Methods Enzymol 200,38-62.

Hubbard, S. R., Wei, L., Ellis, L., and Hendrickson, W. A. (1994). Crystal structure of the tyrosine kinase domain of the human insulin receptor. Nature 372, 746-754.

Jauch, R., Bourenkov, G. P., Chung, H. R., Urlaub, H., Reidt, U., Jackle, H., and Wahl, M. C. (2003). The zinc finger-associated domain of the Drosophila transcription factor 
grauzone is a novel zinc-coordinating protein-protein interaction module. Structure 11, 1393-1402.

Johnson, L. N., Noble, M. E., and Owen, D. J. (1996). Active and inactive protein kinases: structural basis for regulation. Cell $85,149-158$.

Knauf, U., Tschopp, C., and Gram, H. (2001). Negative regulation of protein translation by mitogen-activated protein kinase-interacting kinases 1 and 2. Mol Cell Biol 21, 55005511.

Knighton, D. R., Zheng, J. H., Ten Eyck, L. F., Ashford, V. A., Xuong, N. H., Taylor, S. S., and Sowadski, J. M. (1991a). Crystal structure of the catalytic subunit of cyclic adenosine monophosphate-dependent protein kinase. Science 253, 407-414.

Knighton, D. R., Zheng, J. H., Ten Eyck, L. F., Xuong, N. H., Taylor, S. S., and Sowadski, J. M. (1991b). Structure of a peptide inhibitor bound to the catalytic subunit of cyclic adenosine monophosphate-dependent protein kinase. Science 253, 414-420.

Krishna, S. S., Majumdar, I., and Grishin, N. V. (2003). Structural classification of zinc fingers: survey and summary. Nucleic Acids Res 31, 532-550.

Manning, G., Whyte, D. B., Martinez, R., Hunter, T., and Sudarsanam, S. (2002). The protein kinase complement of the human genome. Science 298, 1912-1934.

McRee, D. E. (1999). XtalView/Xfit--A versatile program for manipulating atomic coordinates and electron density. J Struct Biol 125, 156-165.

Mol, C. D., Fabbro, D., and Hosfield, D. J. (2004). Structural insights into the conformational selectivity of STI-571 and related kinase inhibitors. Curr Opin Drug Discov Devel 7, 639-648.

Nagar, B., Bornmann, W. G., Pellicena, P., Schindler, T., Veach, D. R., Miller, W. T., Clarkson, B., and Kuriyan, J. (2002). Crystal structures of the kinase domain of c-Abl in complex with the small molecule inhibitors PD173955 and imatinib (STI-571). Cancer Res 62, 4236-4243.

Nikolcheva, T., Pyronnet, S., Chou, S. Y., Sonenberg, N., Song, A., Clayberger, C., and Krensky, A. M. (2002). A translational rheostat for RFLAT-1 regulates RANTES expression in T lymphocytes. J Clin Invest 110, 119-126.

Nolen, B., Taylor, S., and Ghosh, G. (2004). Regulation of protein kinases; controlling activity through activation segment conformation. Mol Cell 15, 661-675. 
O'Loghlen, A., Gonzalez, V. M., Pineiro, D., Perez-Morgado, M. I., Salinas, M., and Martin, M. E. (2004). Identification and molecular characterization of Mnk1b, a splice variant of human MAP kinase-interacting kinase Mnk1. Exp Cell Res 299, 343-355.

Otwinowski, Z., and Minor, W. (1997). Processing of X-ray Diffraction Data Collected in Oscillation Mode. Meth Enz 276, 307-326.

Pargellis, C., Tong, L., Churchill, L., Cirillo, P. F., Gilmore, T., Graham, A. G., Grob, P. M., Hickey, E. R., Moss, N., Pav, S., and Regan, J. (2002). Inhibition of p38 MAP kinase by utilizing a novel allosteric binding site. Nat Struct Biol 9, 268-272.

Pyronnet, S., Imataka, H., Gingras, A. C., Fukunaga, R., Hunter, T., and Sonenberg, N. (1999). Human eukaryotic translation initiation factor 4G (eIF4G) recruits mnk1 to phosphorylate eIF4E. Embo J 18, 270-279.

Reiling, J. H., Doepfner, K. T., Hafen, E., and Stocker, H. (2005). Diet-dependent effects of the Drosophila Mnk1/Mnk2 homolog Lk6 on growth via eIF4E. Curr Biol 15, 24-30.

Richter, J. D., and Sonenberg, N. (2005). Regulation of cap-dependent translation by eIF4E inhibitory proteins. Nature $433,477-480$.

Scheper, G. C., Morrice, N. A., Kleijn, M., and Proud, C. G. (2001). The mitogen-activated protein kinase signal-integrating kinase Mnk2 is a eukaryotic initiation factor 4E kinase with high levels of basal activity in mammalian cells. Mol Cell Biol 21, 743754.

Scheper, G. C., Parra, J. L., Wilson, M., Van Kollenburg, B., Vertegaal, A. C., Han, Z. G., and Proud, C. G. (2003). The $\mathrm{N}$ and $\mathrm{C}$ termini of the splice variants of the human mitogenactivated protein kinase-interacting kinase Mnk2 determine activity and localization. Mol Cell Biol 23, 5692-5705.

Seethala, R., and Menzel, R. (1998). A fluorescence polarization competition immunoassay for tyrosine kinases. Anal Biochem 255, 257-262.

Slentz-Kesler, K., Moore, J. T., Lombard, M., Zhang, J., Hollingsworth, R., and Weiner, M. P. (2000). Identification of the human Mnk2 gene (MKNK2) through protein interaction with estrogen receptor beta. Genomics 69, 63-71.

Taylor, S. S., and Radzio-Andzelm, E. (1994). Three protein kinase structures define a common motif. Structure 2, 345-355. 
Tereshko, V., Teplova, M., Brunzelle, J., Watterson, D. M., and Egli, M. (2001). Crystal structures of the catalytic domain of human protein kinase associated with apoptosis and tumor suppression. Nat Struct Biol 8, 899-907.

Ueda, T., Watanabe-Fukunaga, R., Fukuyama, H., Nagata, S., and Fukunaga, R. (2004). Mnk2 and Mnk1 are essential for constitutive and inducible phosphorylation of eukaryotic initiation factor 4E but not for cell growth or development. Mol Cell Biol 24, 65396549.

von der Haar, T., Gross, J. D., Wagner, G., and McCarthy, J. E. (2004). The mRNA capbinding protein eIF4E in post-transcriptional gene expression. Nat Struct Mol Biol 11, 503-511.

Waskiewicz, A. J., Flynn, A., Proud, C. G., and Cooper, J. A. (1997). Mitogen-activated protein kinases activate the serine/threonine kinases Mnk1 and Mnk2. Embo J 16, 1909-1920.

Waskiewicz, A. J., Johnson, J. C., Penn, B., Mahalingam, M., Kimball, S. R., and Cooper, J. A. (1999). Phosphorylation of the cap-binding protein eukaryotic translation initiation factor 4E by protein kinase Mnk1 in vivo. Mol Cell Biol 19, 1871-1880.

Wilson, K. P., Fitzgibbon, M. J., Caron, P. R., Griffith, J. P., Chen, W., McCaffrey, P. G., Chambers, S. P., and Su, M. S. (1996). Crystal structure of p38 mitogen-activated protein kinase. J Biol Chem 271, 27696-27700. 


\section{Chapter II: The MAPK Interacting Kinase (Mnk) Subfamily is Regulated via a Novel Autoinhibitory Mechanism}

\section{Citation:}

Jauch, R., Jäkel, S., Netter, C., Schreiter, K., Aicher, B., Jäckle, H., and Wahl, M. (2005). The MAPK Interacting Kinase (Mnk) Subfamily is Regulated via a Novel Autoinhibitory Mechanism. to be submitted.

\section{Statement of Contribution:}

crystallization, data collection, structure solution, model building, structure refinement, structure analysis, examination of and comparison to available protein kinase structures, manuscript preparation 


\title{
The MAPK Interacting Kinase (Mnk) Subfamily is Regulated via a Novel Autoinhibitory Mechanism
}

\author{
Ralf Jauch $^{1}$, Stefan Jäkel ${ }^{2}$, Catharina Netter ${ }^{3}$, Kay Schreiter ${ }^{2}$, Babette Aicher $^{2}$, Herbert Jäckle ${ }^{1}$ \\ and Markus C. Wahl ${ }^{3}$
}

\author{
Max-Planck-Institut für Biophysikalische Chemie \\ ${ }^{1}$ Abteilung Molekulare Entwicklungsbiologie \\ ${ }^{3}$ Abteilung Zelluläre Biochemie/Röntgenkristallographie \\ Am Faßberg 11 \\ D-37077 Göttingen, Germany \\ ${ }^{2}$ Develogen AG \\ Rudolf-Wissell-Str. 28 \\ D-37079 Göttingen, Germany
}

MAP kinase interacting kinases 1 and 2 (Mnk1/2) regulate the expression of certain genes via phosphorylation of key factors involved in translation initiation. Here we present the crystal structure of the unphosphorylated Mnk1 catalytic domain showing that an otherwise structurally conserved element at the C-terminus of the activation segment of Mnk1 occupies a novel binding site alongside helix $\alpha \mathrm{C}$. This reorganization seeds a circuit of structural rearrangements. Firstly, it causes closing of the interlobal cleft by reorienting the $\mathrm{N}$-terminal lobe through interactions with $\alpha \mathrm{C}$. Secondly, a phenylalanine which derives from a Mnk specific insertion is plunged into a hydrophobic pocket that is normally occupied by the phenylalanine of the Asp-Phe-Gly (DFG) motif (DFD in Mnks) of the Magnesium binding loop. The DFD motif, in turn, flips by almost $180^{\circ}$ and occupies the ATP binding site. This conformation is stabilized by carboxylcarboxyl side chain interactions of the DFD motif with the catalytic aspartate and a conserved glutamate residue of $\alpha \mathrm{C}$. The $\mathrm{C}$-terminal end of the activation segment is therefore employed as an autoinhibitory domain similar to the juxtamembrane domains of c-Kit and FLT3, respectively. Comparison of the Mnk1 structure with Mnk2 allows us to propose a model for the mechanistic dynamics that accompany the activation of Mnk subfamily members. It predicts a novel regulatory principle that is unique for the action of Mnks. 


\section{Introduction}

Mitogen activated protein kinases (MAPK) interacting kinases (Mnk's) comprise a small subfamily of serine-threonine kinases ( Manning et al., 2002; Jauch et al., 2005). Mnk's are activated through phosphorylation by the growth factor stimulated Ras- extracellular signal regulated protein kinases (ERK)1/2 and the stress induced p38-pathway (Fukunaga and Hunter, 1997; Waskiewicz et al., 1997). The two mammalian Mnk isoforms, Mnk1 and Mnk2, phosphorylate the eukaryotic initiation factor 4E (eIF4E) in vitro and in vivo (Waskiewicz et al., 1999; Scheper et al., 2001; Ueda et al., 2004). eIF4E binds the CAP structures of eukaryotic messenger RNA's (Marcotrigiano et al., 1997) suggesting that Mnk dependent control of eIF4E phosphorylation affects CAP dependent translation (Waskiewicz et al., 1999). However, the effect of eIF4E phosphorylation by Mnks on global protein synthesis is under debate (Scheper and Proud, 2002). At the organismic level, it has been shown that mice deficient for Mnk1/2 develop normally implying that Mnks have no essential function (Ueda et al., 2004). However, in Drosophila the single Mnk orthologue, Lk6, was shown to regulate growth, body mass and lipid metabolism (Arquier et al., 2005; Reiling et al., 2005). The Lk6 phenotype was dependent on amino acid availability and has been interpreted to be a result of an intricate interplay with the mTOR pathway (Reiling et al., 2005). Furthermore, a most recent study indicates that Mnks control the translation initiation of specific mRNAs also by phosphorylating different targets (Buxade et al., 2005). This study demonstrates, that phosphorylation of the RNA binding heterogenous nuclear ribonucleoprotein A1 (hnRNPA1) diminishes the translation of the tumor necrosis factor alpha (TNF- $\alpha$ ). Hence, Mnks have the potential to regulate inflammation by controlling the TNF- $\alpha$ levels.

Mnks have also been reported to affect the translation of RFLAT-1 as well as of viral RNAs (Nikolcheva et al., 2002; Walsh and Mohr, 2004). Therefore Mnk mediated phosphorylation appears to regulate the translation of specific mRNAs but the molecular details of the role of Mnks in translation initiation are still unkown. However, the involvement of Mnks in growth control, in lipid metabolism, viral translation and TNF- $\alpha$ regulation defines them as a potential targets for pharmaceutical intervention. 
Recently we reported the crystal structure of the Mnk2 kinase region (Mnk2-KR) (Jauch et al., 2005) showing an extended conformation of the activation segment and a novel zinc coordinating site in a Mnk specific insertion, two features absent from other protein kinases. In addition, we noted the substitution of the highly conserved Magnesium binding Asp-Phe-Gly (DFG) motif by Asp-Phe-Asp (DFD) which adopts a conformation that obstructs ATP binding. However, the significance of the extended activation segment and the trigger for adoption of the inhibitory DFD motif conformation remained elusive. Here we present the crystal structure of the Mnk1 kinase region (Mnk1-KR), showing that it adopts a conformation different from the Mnk2-KR. The Mnk1-KR structure reveals a structurally rearranged activation segment that serves as an autoinhibitory module in the non-phosphorylated form. Buttressing of the activation segment against the N-terminal lobe closes the interlobal cleft and leads to the adoption of an ATP-competitive conformation of the DFD motif. Furthermore the results reveal how the Mnk-specific Asp of the DFD motif stabilizes this autoinhibited state. We propose a model of Mnk activation in which the N-terminal lobe, the Magnesium binding loop and the activation segment undergo distinct structural rearrangements to proceed in a stepwise fashion from an autoinhibited to an active state.

\section{Results}

\section{Structure Solution and overall Architecture of Mnk1-KR}

We produced a polypeptide spanning residues 37 to 341 of Mnk1 in Escherischia coli BL21 cells and purified it to homogeneity. The construct includes the catalytic domain of Mnk-1 and is henceforth referred to as Mnk1 kinase region (Mnk1-KR). As shown for Mnk2KR (Jauch et al., 2005) this fragment recapitulates the activity of the full length protein, i.e. it phosphorylates a eIF4E peptide after activation by ERK2 in vitro ( Mnk1 data not shown). Needlelike, tetragonal crystals of Mnk1-KR could be grown at $20^{\circ} \mathrm{C}$ using the vapor diffusion method. A 2.8 dataset was collected at the Swiss-Light-Source (Table 1). After molecular replacement using a truncated model of the Mnk2-KR (Jauch et al., 2005) followed by density modification, an interpretable electron density was obtained. The model could subsequently be refined to $R / R_{\text {free }}$ factors of $23.5 / 28.0 \%$ (Table 1 ). The asymmetric unit contains two Mnk1- 

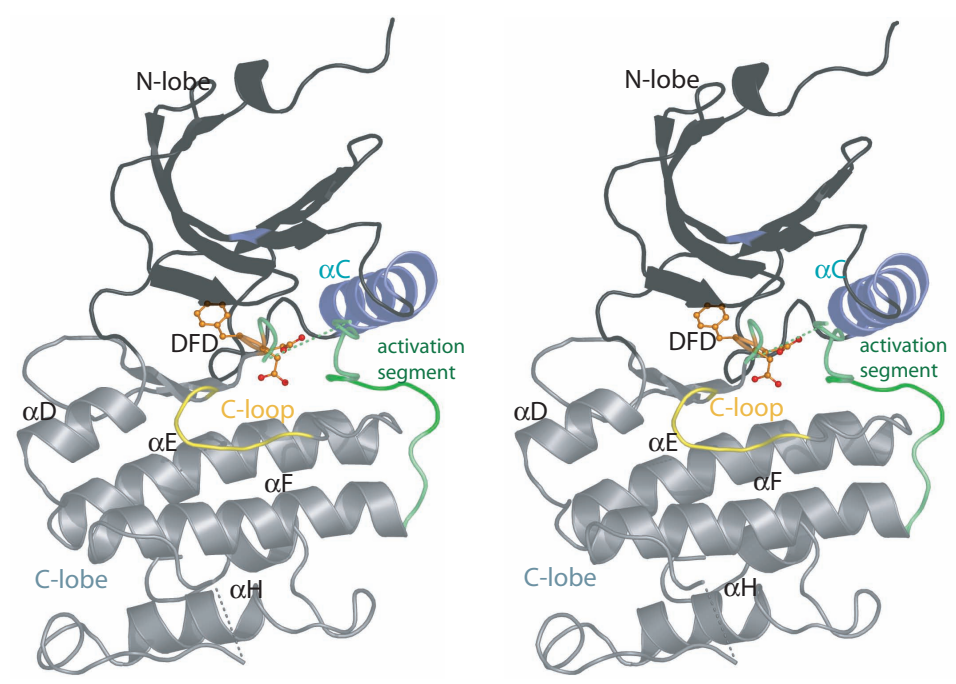

(A)

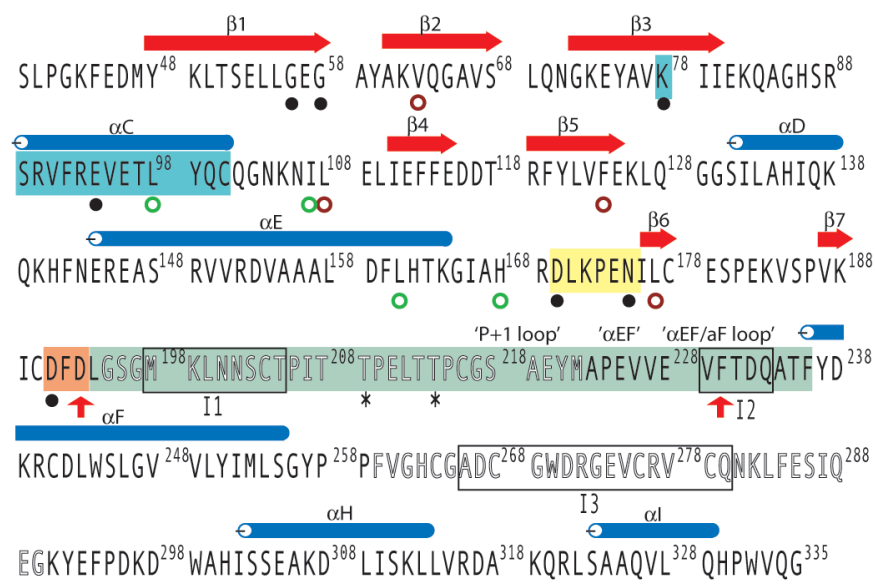

(B)

Figure 1: Overall structure of Mnk1 in stereo representation (A) and primary sequence (B). If not indicated otherwise the coloring scheme is maintained in the following figures: N-lobe: black; C-lobe: gray; C-loop: yellow; DFG/D-motif orange; $\alpha \mathrm{C}$ helix and Lys-Glu pair: blue; activation segment: green. Connections which could not be traced in the $2 \mathrm{Fo}-\mathrm{Fc}$ electron density are indicated with dotted lines. (B) residues known to interact with ATP are marked with closed circles, residues comprising the DFG/D-in pocket: green empty circles; residues comprising the DFG/D-out pocket: red empty circles. Mnk specific amino acid insertions are boxed and Mnk specific residues with functional relevance are highlighted with a red arrow. Phosphorylation sites are indicated with stars. Regions expected to form conserved secondary structure elements in the active state are show with quotation marks: 'P +1 loop', ' $\alpha E F$ ' and the ' $\alpha E F / \alpha F$ loop'. Note that those elements are not present in their functional configuration in the inactive Mnk1 structure. Regions not traced in the $2 \mathrm{Fo}-\mathrm{Fc}$ electron density are shown as empty letters. 
KR molecules which are related by a non-crystallographic two-fold axis. Molecule A exhibits lower temperature factors and a clearer electron density in several regions. The functionally important regions, however, are virtually identical in molecule A and molecule B. The final model spans residues 39-335 of Mnk1.

The Mnk1-KR preserves several global features of protein kinase (PK) architecture including the bilobal makeup (Knighton et al., 1991a). The N-terminal lobe contains a twisted sheet of five antiparallel $\beta$-strands $(\beta 1-\beta 5)$ and the regulatory helix $\alpha \mathrm{C}$ (Figure 1). It contains the key elements essential for ATP binding such as the Gly rich loop, the Lys78 from $\beta 3$ and the Glu94 emanating from $\alpha \mathrm{C}$. The larger C-terminal lobe is predominantly $\alpha$-helical and contains the elements required for substrate binding and phosphate transfer. It includes the catalytic loop (C-loop, residues 170-175), the DFD motif (residues 191-193) and the activation segment (Figure 1). Two segments within the Mnk1-KR exhibit strong conformational flexibility and could thus not be traced in the electron density. The first segment comprises the core of the activation segment including the activation loop and the $\mathrm{P}+1$ loop (residues 197222). The second segment spans the Mnk specific cysteine cluster and helix $\alpha \mathrm{G}$ (residues 261290).

\section{The activation segment reorients the $\mathbf{N}$-lobe via helix $\alpha C$}

In its canonical conformation as seen in most other protein kinases the $\mathrm{P}+1$ loop as well as the succeeding helix $\alpha-E F$ are buried within the kinase core in an environment provided by the catalytic loop and the helices $\alpha \mathrm{F}, \alpha \mathrm{G}$ (Knighton et al., 1991a). As a result, the $\mathrm{P}+1$ loop is in a position to interact with the peptide substrate, e.g. with the residue downstream of the phophorylation site (hence the name $\mathrm{P}+1$ loop). The short helix $\alpha$-EF is connected to helix $\alpha \mathrm{F}$ through the $\alpha-\mathrm{EF} / \alpha \mathrm{F}$ loop which varies in size and primary sequence between individual PKs (Nolen et al., 2004). In contrast, the region corresponding to $\alpha$-EF and the $\alpha-E F / \alpha F$ loop of the Mnk1-KR adopts a different conformation. This region is unwound and traverses the cleft between the N-terminal and C-terminal lobes. This way, the residues of the highly conserved Asp-Pro-Glu (APE) motif that usually form the helix $\alpha$-EF come to lie at the N-terminal lobe, lining up with helix $\alpha \mathrm{C}$ (Figure 2A). This conformation is stabilized by interaction of Glu225 (APE motif) and Glu228 ( $\alpha$-EF/ $\alpha$ F loop) with Arg90 and Arg93 form $\alpha \mathrm{C}$. Significantly, residues corresponding to Arg90 and Arg93 are often bound to phosphate 

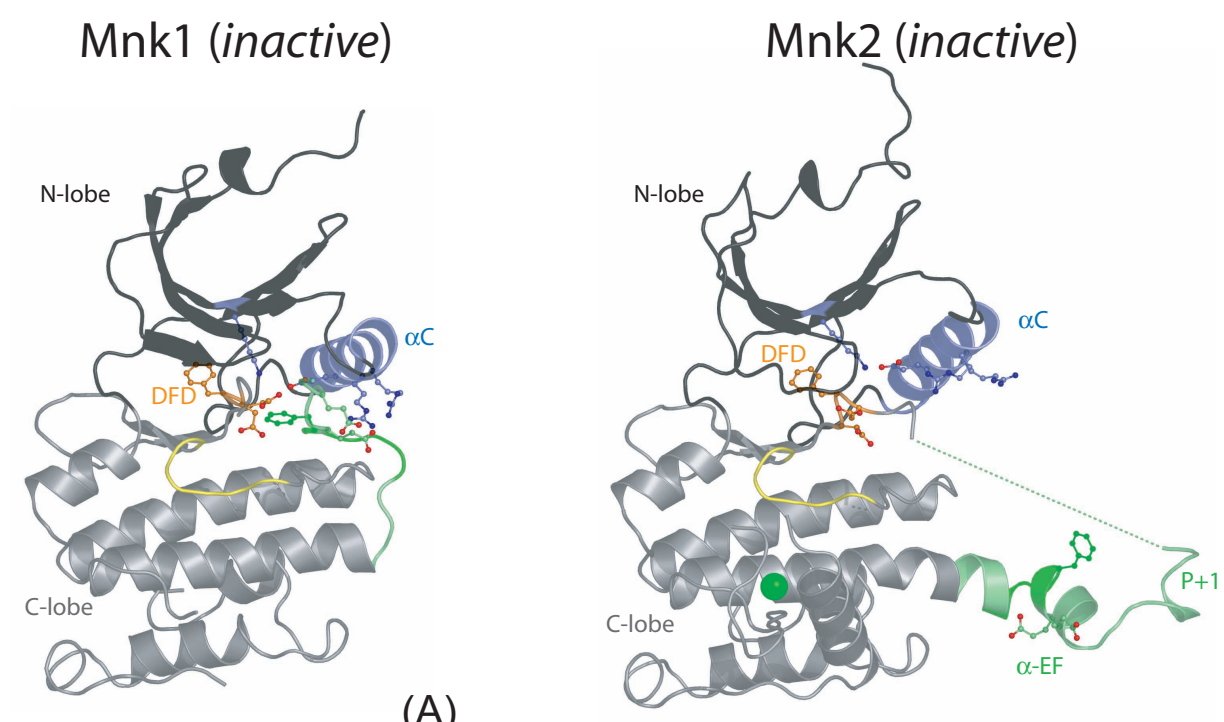

(B)

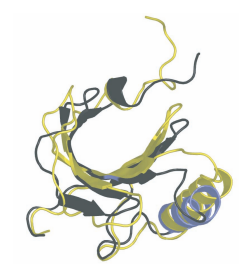

Mnk1

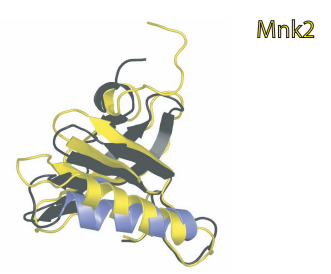

(D)

Figure 2: N-lobe movement induced by the activation segment. Overall structure of Mnk1 (A) and Mnk2 (B). The DFD-motif, Phe-230, Arg-90, Arg-93, Glu225 and Glu-228 are shown in the ball-and-stick representation (Mnk1 numbering). Arg90 and Arg93 correspond to residues known to interact with phospho amino acids (Krupa et al., 2004). (C, D) Comparison of the N-lobes of Mnk1 (black, $\alpha \mathrm{C}$ blue) and Mnk2 (yellow) after the superposition of the C-lobes (C-lobes not shown). (C) orientation as in (A,B). (D) rotated by $90^{\circ}$ around the vertical axis. 
moieties in active protein kinases (Krupa et al., 2004). Therefore, in inactive Mnk1, the region corresponding to $\alpha-\mathrm{EF}$ and the $\alpha-\mathrm{EF} / \alpha \mathrm{F}$ loop is apparently positioned next to helix $\alpha \mathrm{C}$ by residues which in active PKs engage in alternative interactions. This observation suggests that phosphorylation will provide a trigger that releases the activation segment from helix $\alpha \mathrm{C}$.

As a consequence of the unusual positioning of the $\alpha-E F-\alpha-E F / \alpha F$ loop region, helix $\alpha \mathrm{C}$ and the remainder of the $\mathrm{N}$-terminal lobe are displaced towards the $\mathrm{C}$-terminal lobe and thereby narrow the interlobal cleft (Figure 2A). Thus, in the Mnk1 structure, the structural region corresponding to the $\mathrm{P}+1$ loop and $\alpha-\mathrm{EF}$ adopts a conformation that is incompatible with catalysis and must therefore be rearranged for activity. Hence, the activation segment for Mnks spans the region located between the DFD motif and helix $\alpha \mathrm{F}$. It therefore includes, in contrast to previous conventions (Nolen et al., 2004), $\alpha$-EF and the prolonged $\alpha-E F / \alpha F$ loop (Figure 1).

The unusual conformation of the activation segment of Mnk1 may be the result of two amino acid insertions which are not present in most other $\mathrm{Ca}^{2+} / \mathrm{Calmodulin}$ modulated kinases (CAMK) group PKs. Insertion 1 (I1, Figure 1) is located C-terminal of the DFG/D-motif and is disordered in the present structure. Insertion 2 (I2) affects a region corresponding to the $\alpha$ $\mathrm{EF} / \alpha \mathrm{F}$ loop. Notably, residues of insertion $\mathrm{I} 2$ participate in the stabilization of the conformation seen in Mnk1 (e.g. Glu228 and Phe230).

\section{The activation segment induces an ATP-competitive conformation}

The vast majority of protein kinases bears an DFG motif at the beginning of the activation segment (subdomain VII) which shapes the 'lip' of the ATP binding 'mouth' of protein kinases at the interlobal cleft (Hanks, 2003; Hanks et al., 1988; Taylor and RadzioAndzelm, 1994). The first aspartate of this motif is invariant among catalytically active protein kinases and is known to coordinate a magnesium ion that is essential for phosphoryl transfer (Adams, 2001). The region encompassing the DFG motif has therefore been termed the magnesium binding loop. Mnks contain a DFD motif at the corresponding position. We thus refer to the motif as DFG/D. In active kinases, the Phe of the DFG/D is situated in a structurally conserved pocket. In Mnk1, this pocket is provided by Leu98 and Thr97 
(emanating from $\alpha \mathrm{C}$ ), His168 (upstream of the C-loop), Ile107, Ile189 and Leu161. This active DFG/D motif conformation is referred to as DFG/D-in conformation and its corresponding binding site will henceforth be referred to as DFG/D-in pocket.

The conformation of the activation segment as seen in the present Mnk1 structure has a dramatic effect on the DFD. As a result of the activation segment repositioning Phe230, comes to lie in the DFG/D-in pocket (Figure 3). It thereby displaces the DFGD. The DFD motif is in turn rotated by $\sim 180^{\circ}$ around the $\Phi$ angle of Asp191 $\left(\Phi_{\mathrm{Asp} 191}=-120\right)$ with respect to the DFG/D-in conformation of active state protein kinases (e.g. DAPK1 $\Phi_{\mathrm{Asp} 161}=55^{\circ}$; Figure $4 \mathrm{~A}$; Tereshko et al., 2001). As a result the Phe of the DFG/D (Phe192) occupies a hydrophobic pocket provided by Val63, Leu108, the 'gatekeeper residue' Phe124 and Leu177 which would normally accommodate the adenosyl moiety of ATP. The resulting DFG/D conformation is referred to as DFG/D-out. The DFG/D-out conformation has also been described for Mnk2 (Jauch et al., 2005).

\section{The Mnk-specific DFD stabilizes the DFD-out Conformation}

The DFD motif of Mnk1 participates in a network of polar interactions that explains the preference for the DFG/D-out conformation. Both, the invariant Asp191 and the Mnk specific Asp193 are engaged in tight carboxyl-carboxyl side chain interactions with active site residues (Figure 4B): (i) Asp191 binds to Glu93; (ii) Asp193 binds to Asp170. Glu94 and Asp170 correspond to residues which are invariant among catalytically active protein kinases (Hanks et al., 1988). Glu-94 emanates from the regulatory helix $\alpha \mathrm{C}$ and is known to form an ion pair with Lys78 that is essential for productive ATP binding (Adams, 2001). This pairing is obstructed in Mnk1, since Asp191 of the DFG/D motif interacts with Glu94 (ODAsp191:OE-Glu94) and with Lys78 (O-Asp191:Nz-Lys78). Asp170, which interacts with Asp193, corresponds to the catalytic aspartate of the C-loop.

Although carboxyl-carboxyl side chain interactions appear unusual, they have often been observed in proteins structures and are particularly abundant within the catalytic centers of enzymes (Flocco and Mowbray, 1995). The pH of the crystallization conditions for Mnk1 (pH 5.6) may have favored the stabilization of those interaction. However, acid-acid interactions have been observed even at basic buffer conditions (Flocco and Mowbray, 1995). The O-O distances between the two carboxylic acid groups reside in proximity of $2.6 \AA$ and 

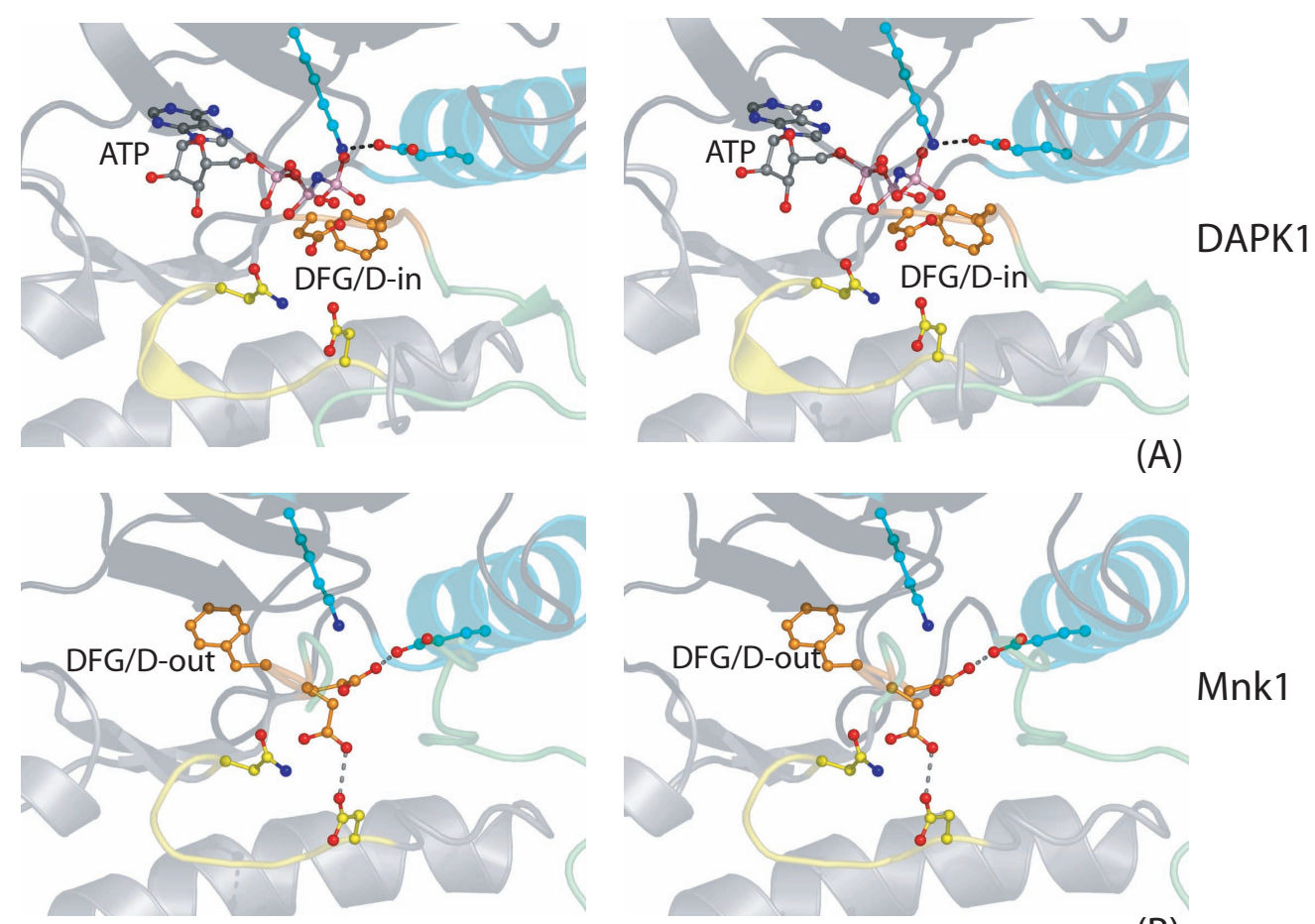

(B)

Figure 3: The ATP binding pocket of active DAPK1 (A; PDB-id: 1ig1; (Tereshko et al., 2001)) and inactive Mnk1 (B). The molecules are in the same orientation as in Fig. 1 with the ATP binding regions blown up. (A) active DAPK1 contains the non-cleavable ATP analogon ANP-PNP (ball-and-stick) at the functional site. Note the permissive DFG/D-in conformation of the Magnesium binding DFG-motif. The ATP site blockade of Mnk1 (B) and Mnk2 (Jauch et al., 2005) is achieved by the inhibitory DFG/D-out conformation. The DFD-motif, Lys78, Glu94, Asp-170, Asn-175 and corresponding residues of DAPK1 are shown in the ball-and-stick representation. 
$2.5 \AA$ which is significantly shorter than the average O-O distance between non-acidic hydrogen donor/acceptor pairs. The latter observation was attributed to a proton sharing binding mode (Flocco and Mowbray, 1995). As described for other such carboxyl-carboxyl interactions Asp191:Glu94 as well as Asp193:Asp170 are stabilized by amines (Lys78) or amides (Asn175), respectively (see e.g. (Werten et al., 2002) for comparison).

\section{Discussion}

\section{The activation segment of Mnk1 is reorganized}

Previous studies have shown that in most protein kinases, the flexible portion of the activation segment is restricted to the activation loop, which is located between the DFG/D motif and the $\mathrm{P}+1$ loop (Nolen et al., 2004). The $\mathrm{P}+1$ loop interacts with the residue adjacent to the phosphorylation site of substrate peptides and therefore plays an important role in substrate peptide positioning (Knighton et al., 1991b). Within the Mnk subfamily, however, the activation segment is extended through two amino acid insertions (Figure 1). In addition, the region subject to structural rearrangements is strongly expanded since the flexible portion not only includes the activation loop but also the succeeding $\mathrm{P}+1$ loop, the region corresponding to the short helix $\alpha-E F$ and the $\alpha-E F / \alpha F$ loop. Both the $\mathrm{P}+1$ loop and the $\alpha-E F$ helix occupy conserved sites within the vast majority of reported protein kinase structures, no matter whether the active or the inactive version of the enzymes were crystallized (Nolen et al., 2004). In contrast, the $\alpha$-EF helix and the $\alpha-E F / \alpha F$ loop of Mnk1 unfold and traverse the interlobal cleft. Hence, the activation segment of Mnk1 is not only longer as compared to other PKs but also structurally rearranged in an unprecedented manner. This observation has profound consequences for the function of Mnk1.

\section{Variegation of the Juxtamembrane Theme: Autoinhibtion of Mnk1}

The previously reported Mnk2 structure displays an inactive conformation which is characterized by the ATP competitive DFG/D-out conformation (Jauch et al., 2005). The autoinhibition could be destabilized in Mnk2 by introducing the canonical DFG motif in place 


\section{c-KIT (autoinhibited)}

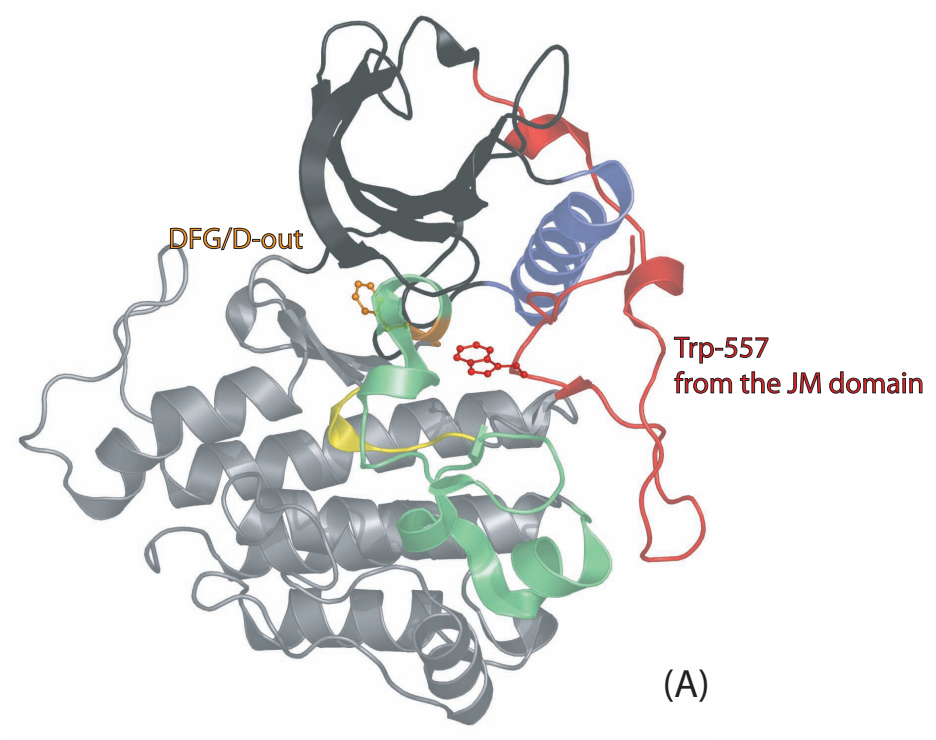

Mnk1 (autoinhibited)

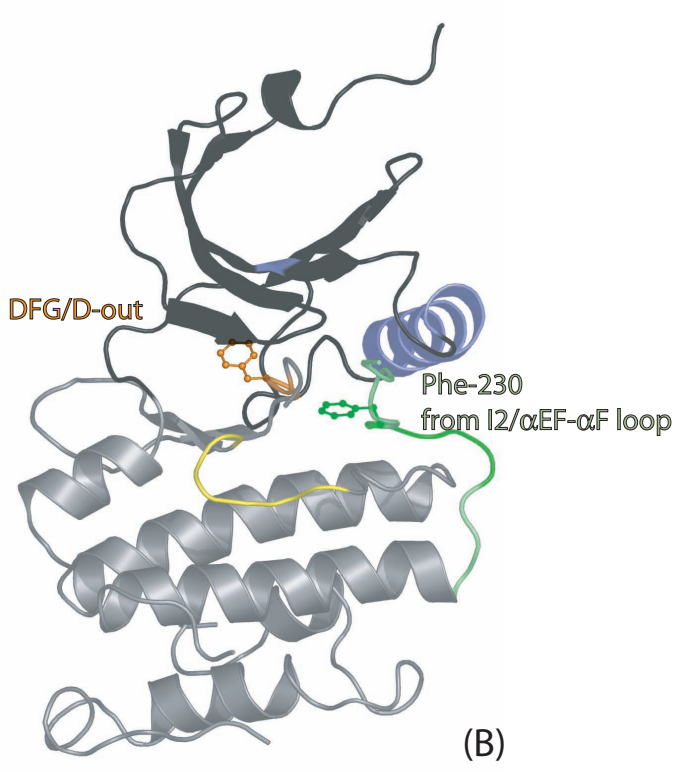

Figure 4: Autoinhibtion in c-KIT (A; PDB-id: 1t45) and Mnk1 (B). The autoinhibitory JM domain of c-KIT crosses the interlobal cleft coming from the $\mathrm{N}$-terminus of the molecule (colored red). A region corresponding to helix $\alpha E F$ and the $\alpha E F / \alpha F$ loop traverses the interlobal cleft in the opposite direction in Mnk1. Residues occupying the DFG/D-in pocket and the Phe of the DFG/D-motif are displayed in the ball-and-stick representation. Corresponding structural elements are colored equally. Note that the activation segment of c-KIT is fully visible in contrast to Mnk1. 
of the Mnk specific DFD motif, suggesting a role of the Mnk-specific Asp. However, the reason for adopting DFG/D-out remained largely speculative in the case of Mnk2. The present Mnk1 structure sheds light on the structural basis for Mnk regulation and clarifies the role of the DFD motif.

Autoinhibition is a prominent strategy for protein kinase regulation which is imposed differently in individual cases and may affect various functional sites of the molecules (Huse and Kuriyan, 2002). Autoinhibiting regulatory domains outside the core of the protein kinase domain are employed, for example, in CAMKI (Goldberg et al., 1996), Twitchin (Kobe et al., 1996), c-KIT (Mol et al., 2004b) and Flt3 (Griffith et al., 2004). In the case of c-KIT and FLT3, two receptor tyrosine kinases of the platelet derived growth factor receptor (PDGFR) family, the autoinhibition is due to a juxtamembrane (JM) domain. This domain connects the transmembrane helix to the cytoplasmatic catalytic domain and functions as a regulatory element in many receptor tyrosine kinases (Hubbard, 2004). In c-KIT and FLT3, the JM domain causes autoinhibition by plunging a hydrophobic residue into the DFG/D-in pocket. As a result, the DFG/D-out conformation is adopted and catalytic activity is repressed (Figure 3; Griffith et al., 2004; Mol et al., 2004a). Although Mnk1 does not contain a JM domain, it is likewise autoinhibited by the induction of the DFG/D-out conformation (Figure 3, 4). The 'molecular trick' employed by Mnk1 parallels the mechanism described for c-KIT and FLT3. In contrast to c-KIT and Flt-3, however, Mnk1 does not bear an autoinhibitory domain outside the PK domain. Instead, Mnk1 induces DFG/D-out through its activation segment. To this end, Mnk1 inserts Phe230 emanating form the Mnk-specific insertion I2 into the DFG/D-in pocket. As a result, the DFG/D-in pocket is inaccessible for the DFG/D motif and the DFG/D-out conformation is enforced. The reorganized activation segment, in particular Phe230, may therefore constitute an autoinhibitory element which plays a key role for a Mnk specific regulatory mechanism. This element serves formally the same function as the JM domain of PDGFRs.

\section{The Ins and Outs of DFG/D}

Both Mnk1 and Mnk2 display the DFG/D-out conformation, which excludes ATP from entering its binding pocket. This observation suggests that the DFG/D-out conformation is a default state of inactive Mnk kinases. The Mnk1 structure provides an explanation for the 
consequences of the Mnk specific Asp within the DFG/D-motif. This Asp interacts with catalytically important residues and thus stabilizes the DFG/D-out conformation (Figure 3). Hence, the DFD motif may facilitate the specific regulation of Mnks by stabilizing an inactive conformation.

Our observation highlights a possible significance of the DFG/D-motif for PK regulation. To date, the DFG/D-out conformation has been observed in only a fraction of the more than 50 protein kinases for which structural data are available (Mol et al., 2004b; Vieth et al., 2005). In some cases, the DFG/D-out conformation has been induced and/or stabilized by the binding of selective inhibitors such as in the structures of Birb796 bound to p38 (Pargellis et al., 2002), Cleevec bound to c-Abl (Nagar et al., 2002) and AAL-993 bound to VEGFR-2 (Manley et al., 2004). Notably, inhibitors targeting the DFG/D-out conformation are expected to bind with higher selectivity when compared to classical compounds which target the ATP binding pocket directly (Mol et al., 2004b). Hence, Mnks display a selective binding surface for specific inhibitors in their unphosphorylated state. The selectivity of this site is further pronounced by the presence of the Mnk specific Asp within the DFG/D motif.

\section{The Mnk1 structure differs from Mnk2}

Although their primary sequence is largely identical the crystal structures of Mnk1 and Mnk2 display pronounced differences in structural modules which are essential for kinase function (Figure 2,4). These differences include (i) the conformation of the activation segment, (ii) the orientation of the two lobes towards each other and (iii) atomic details of the DFG/D-out conformation (Figure 2, 4). In the Mnk2 structure the activation segment protrudes from the PK core and helix $\alpha \mathrm{F}$ is prolonged by one turn. Mnk2 does also display the $\mathrm{P}+1$ loop and helix $\alpha-E F$, a region which is either disordered or structurally rearranged in Mnk1. However, the $\mathrm{P}+1$ loop and $\alpha-\mathrm{EF}$ are inserted into the symmetry equivalent neighbor molecules instead of becoming positioned at their canonical intramolecular binding site (Jauch et al., 2005). As a consequence, the interaction between the activation segment and the N-lobe is relieved and the occupation of the DFG/D-in pocket is reversed in Mnk2. As a result, the Nlobe of Mnk2 could snap back, the interlobal cleft could open up and the Lys-Glu ion pair would be formed (Figure 2B). When compared with Mnk1, the N-lobe of Mnk2 is tilted by approximately $10^{\circ}$ leading to an opening of the ATP binding mouth of the kinase (Figure $2 \mathrm{C}$, 
D). A N-lobe movement would explain the absence of the polar interactions of the DFD motif in the Mnk2 structure.

\section{The mechanistic dynamics of Mnks}

Mnk1 and Mnk2 exhibit a sequence identity of 78\% within their catalytic domains and their cellular function is largely redundant ( Waskiewicz et al., 1999; Scheper et al., 2001). Hence, we expect that they behave similarly at the atomic level. We therefore assume that both structures provide snapshots of different structural states. These states are common among the Mnk subfamily members and can therefore be adopted by Mnk1 and Mnk2. Based on this assumption, we propose a model describing the structural basis for Mnk regulation. The model comprises 3 states: (I) the inhibited state, (II) the intermediate state and (III) the active state (Figure 5). State I is represented by the structure of Mnk1. In this state the PK is autoinhibited through a novel positioning of the reorganized activation segment. It displays an activation segment which traverses the interlobal cleft and aligns with helix $\alpha \mathrm{C}$. As a result DFG/D-out is adopted and the interlobal cleft is closed. Several basic residues which are known to interact with phospho-residues in activated PKs engage in unique intramolecular contacts in state I. First, the Arg upstream of the catalytic loop (RD-Arg) undergoes a polar sidechain interaction with Asp-238 (Asp-273 in Mnk2). Second, basic residues from $\alpha \mathrm{C}$ (Arg-90 and Arg-93) interact with the repositioned activation segment. Both, the RD-Arg and basic residues from $\alpha \mathrm{C}$ are known to form the so-called RD-pocket which accommodates phospho-residues emanating from the activation segment (Krupa et al., 2004; Nolen et al., 2004). The interaction between such phospho-residues and the RD-pocket has been reported to have at least two effects: it positions the activation segment for substrate binding and it induces a closed conformation by re-orientating the N-lobe (Nolen et al., 2004).

It is well established that Mnk's require phosphorylation at two sites within the activation loop to become activated (Waskiewicz et al., 1999). Phosphorylation is expected to disrupt the intramolecular interactions of RD-pocket residues as seen in state I. It is therefore likely that state I is destabilized in favor of the states II/III as a result of such phosphorylation events. We assume that state II, which is depicted by the structure of Mnk2, constitutes an intermediate state on the way to full activation (Jauch et al., 2005). The fact that state II is adopted by Mnk2 in the absence of phosphorylation can be explained by the unusual crystal 


\section{autoinhibited}

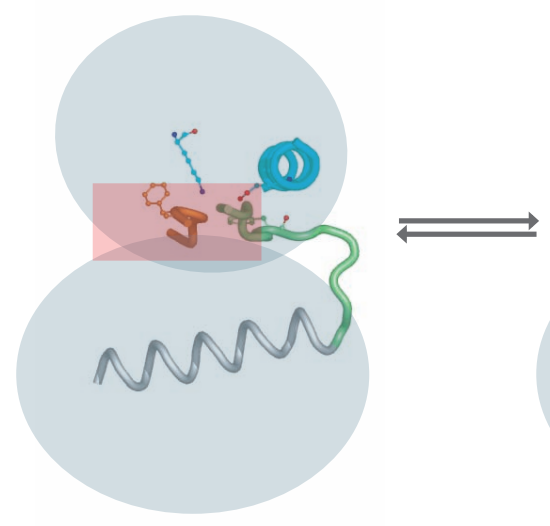

intermediate

active

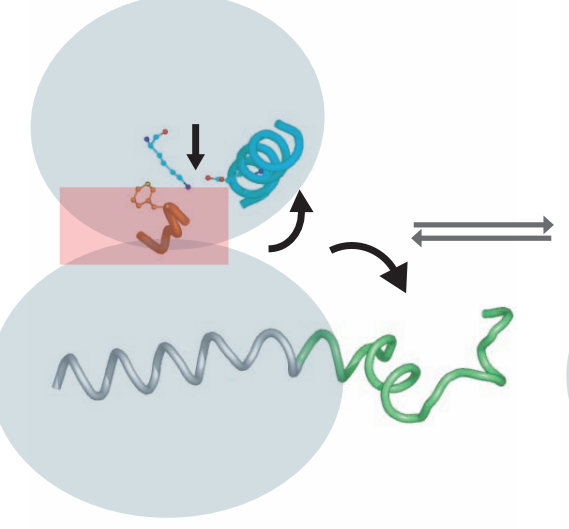

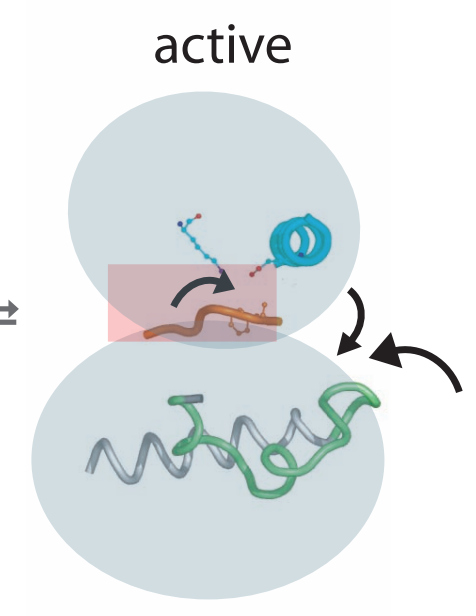

Figure 5: A model of the Mnk activation cascade. The different states are depicted schematically. The N- and $\mathrm{C}$-terminal lobes are indicated with oval circles. The ATP binding site is highlighted with a transparent red box. $\alpha \mathrm{C}$ (blue) and $\alpha \mathrm{F}$ (gray) as well as the magnesium binding loop (orange) and the region corresponding to $\mathrm{P}+1$ (state 2 and 3 only), $\alpha \mathrm{EF}$ and the $\alpha \mathrm{EF} / \alpha \mathrm{F}$ loop (green) are shown as cartoon. The Phe of the DFG/D-motif and the catalytically important Lys-Glu pair are shown as ball-and-stick. Arrows indicate movements of structural elements (see text for details of the model). 
packing interaction. Despite this consideration, state II illustrates the structural consequences which occur, when the activation segment leaves its binding site seen in state I: On the one hand, the N-lobe is subjected to a pronounced reorientation leading to lobe opening and the formation of the Lys-Glu ion pair. On the other hand, the DFG/D-in pocket is no longer occupied by Phe-230. However, state II still displays several features of inactive state kinases.

For the adoption of the active state III, further structural rearrangements are required. Firstly, to enable productive ATP binding, the DFG/D-motif has to adopt the DFG/D-in conformation. This switch has in parts been observed in the previously reported structure of the Mnk2 $2^{\mathrm{D} 228 \mathrm{G}}$ active site mutant (Jauch et al., 2005). Secondly, an inward switch of the P+1 loop and $\alpha-E F$ is required. Without this switch, the canonical mode of peptide substrate binding as described for many PKs can not be pursued ( Hubbard, 1997; Brown et al., 1999; Yang et al., 2002). We further assume a re-closure of the interlobal cleft which brings N-lobe residues such as the Gly rich loop and the Lys-Glu pair to their catalytic position. The lobe reclosure is probably induced by interactions of phospo-residues with RD-pocket residues from $\alpha \mathrm{C}$.

\section{Experimental Procedures}

\section{Cloning and Protein Preparation}

A cDNA fragment of human Mnk-1, corresponding to amino acid residues 37 to 341 and encompassing the kinase domain (KD) was amplified using the forward/reverse primer pair 5'CGGGATCCACTGACTCCTTGCCAGGAAAG/

5'ACGCGTCGACCTATCCCTTTTCTGGAGCTTGCC (utilized restriction sites underlined) and was cloned into the BamHI and SalI sites of the vector pGEX-4T1 (Amersham, Sweden, cat. no. 27-4580-01). This construct allows prokaryotic expression of Mnk1-KR as a fusion protein with a N-terminal, thrombin cleavable glutathione S-transferase (GST) tag. The expression and purification of the Mnk-1KR was essentially identical to method described for Mnk2-KR (Jauch et al., 2005). 


\section{Crystallization and Data Collection}

Needlelike crystals of wild type Mnk1-KR were grown at $20^{\circ} \mathrm{C}$ by vapor diffusion with a reservoir solution containing 20\% (w/v) PEG3350, 0.2M Ammonium sulfate and $0.1 \mathrm{M} \mathrm{Na-}$ Citrate, pH 5.6 and were frozen (liquid nitrogen) in reservoir solution supplemented with $20 \%$ glycerol. Diffraction data (Table 1) were collected on the PXII beamline (SLS, Villingen, Switzerland) at $100 \mathrm{~K}$ on a MarResearch (Norderstedt, Germany) CCD detector and processed with the HKL package (Otwinowski and Minor, 1997).

\section{Structure Solution and Refinement}

The Mnk1-KR structure was solved by molecular replacement (MolRep; (Collaborative Computational Project, 1994) using a truncated version of Mnk2-KR as the search model. The resulting electron density was further modified by density modification and noncrystallographic symmetry (NCS) averaging. Model building was performed manually by interpreting the 2Fo-Fc electron density using Xfit (McRee, 1999). Refinement was performed with the CNS program excluding NCS restraints (Brunger et al., 1998). All data between 15.0 and $2.8 \AA$ resolution were employed in the refinement, setting aside $5 \%$ of the reflections to monitor the free R-factor (Table 1). The molecular figures were prepared with PyMol (http://pymol.sourceforge.net/). 


\section{References}

Adams, J. A. (2001). Kinetic and catalytic mechanisms of protein kinases. Chem Rev 101, 2271-2290.

Arquier, N., Bourouis, M., Colombani, J., and Leopold, P. (2005). Drosophila Lk6 kinase controls phosphorylation of eukaryotic translation initiation factor $4 \mathrm{E}$ and promotes normal growth and development. Curr Biol 15, 19-23.

Brown, N. R., Noble, M. E., Endicott, J. A., and Johnson, L. N. (1999). The structural basis for specificity of substrate and recruitment peptides for cyclin-dependent kinases. Nat Cell Biol 1, 438-443.

Brunger, A. T., Adams, P. D., Clore, G. M., DeLano, W. L., Gros, P., Grosse-Kunstleve, R. W., Jiang, J. S., Kuszewski, J., Nilges, M., Pannu, N. S., et al. (1998). Crystallography \& NMR system: A new software suite for macromolecular structure determination. Acta Crystallographica Section D-Biological Crystallography 54, 905-921.

Buxade, M., Parra, J. L., Rousseau, S., Shpiro, N., Marquez, R., Morrice, N., Bain, J., Espel, E., and Proud, C. G. (2005). The Mnks Are Novel Components in the Control of TNFalpha Biosynthesis and Phosphorylate and Regulate hnRNP A1. Immunity 23, 177-189.

Collaborative Computational Project, N. (1994). The CCP4 Suite: Programs for protein crystallography. Acta Crystallographica D 50, 760-763.

Flocco, M. M., and Mowbray, S. L. (1995). Strange bedfellows: interactions between acidic side-chains in proteins. J Mol Biol 254, 96-105.

Fukunaga, R., and Hunter, T. (1997). MNK1, a new MAP kinase-activated protein kinase, isolated by a novel expression screening method for identifying protein kinase substrates. Embo J 16, 1921-1933.

Goldberg, J., Nairn, A. C., and Kuriyan, J. (1996). Structural basis for the autoinhibition of calcium/calmodulin-dependent protein kinase I. Cell 84, 875-887.

Griffith, J., Black, J., Faerman, C., Swenson, L., Wynn, M., Lu, F., Lippke, J., and Saxena, K. (2004). The structural basis for autoinhibition of FLT3 by the juxtamembrane domain. Mol Cell 13, 169-178. 
Hanks, S. K. (2003). Genomic analysis of the eukaryotic protein kinase superfamily: a perspective. Genome Biol 4, 111.

Hanks, S. K., Quinn, A. M., and Hunter, T. (1988). The protein kinase family: conserved features and deduced phylogeny of the catalytic domains. Science 241, 42-52.

Hubbard, S. R. (1997). Crystal structure of the activated insulin receptor tyrosine kinase in complex with peptide substrate and ATP analog. Embo J 16, 5572-5581.

Hubbard, S. R. (2004). Juxtamembrane autoinhibition in receptor tyrosine kinases. Nat Rev Mol Cell Biol 5, 464-471.

Huse, M., and Kuriyan, J. (2002). The conformational plasticity of protein kinases. Cell 109, 275-282.

Jauch, R., Jäkel, S., Netter, C., Schreiter, K., Aicher, B., Jäckle, H., and Wahl, M. (2005). Crystal Structures of the Mnk2 Kinase Domain Reveal an Inhibitory Conformation and a Zinc-Binding Site. Structure.

Knighton, D. R., Zheng, J. H., Ten Eyck, L. F., Ashford, V. A., Xuong, N. H., Taylor, S. S., and Sowadski, J. M. (1991a). Crystal structure of the catalytic subunit of cyclic adenosine monophosphate-dependent protein kinase. Science 253, 407-414.

Knighton, D. R., Zheng, J. H., Ten Eyck, L. F., Xuong, N. H., Taylor, S. S., and Sowadski, J. M. (1991b). Structure of a peptide inhibitor bound to the catalytic subunit of cyclic adenosine monophosphate-dependent protein kinase. Science 253, 414-420.

Kobe, B., Heierhorst, J., Feil, S. C., Parker, M. W., Benian, G. M., Weiss, K. R., and Kemp, B. E. (1996). Giant protein kinases: domain interactions and structural basis of autoregulation. Embo J 15, 6810-6821.

Krupa, A., Preethi, G., and Srinivasan, N. (2004). Structural modes of stabilization of permissive phosphorylation sites in protein kinases: distinct strategies in Ser/Thr and Tyr kinases. J Mol Biol 339, 1025-1039.

Manley, P. W., Bold, G., Bruggen, J., Fendrich, G., Furet, P., Mestan, J., Schnell, C., Stolz, B., Meyer, T., Meyhack, B., et al. (2004). Advances in the structural biology, design and clinical development of VEGF-R kinase inhibitors for the treatment of angiogenesis. Biochim Biophys Acta 1697, 17-27. 
Manning, G., Whyte, D. B., Martinez, R., Hunter, T., and Sudarsanam, S. (2002). The protein kinase complement of the human genome. Science 298, 1912-1934.

Marcotrigiano, J., Gingras, A. C., Sonenberg, N., and Burley, S. K. (1997). Cocrystal structure of the messenger RNA 5' cap-binding protein (eIF4E) bound to 7-methyl-GDP. Cell $89,951-961$.

McRee, D. E. (1999). XtalView/Xfit--A versatile program for manipulating atomic coordinates and electron density. J Struct Biol 125, 156-165.

Mol, C. D., Dougan, D. R., Schneider, T. R., Skene, R. J., Kraus, M. L., Scheibe, D. N., Snell, G. P., Zou, H., Sang, B. C., and Wilson, K. P. (2004a). Structural basis for the autoinhibition and STI-571 inhibition of c-Kit tyrosine kinase. J Biol Chem 279, 31655-31663.

Mol, C. D., Fabbro, D., and Hosfield, D. J. (2004b). Structural insights into the conformational selectivity of STI-571 and related kinase inhibitors. Curr Opin Drug Discov Devel 7, 639-648.

Nagar, B., Bornmann, W. G., Pellicena, P., Schindler, T., Veach, D. R., Miller, W. T., Clarkson, B., and Kuriyan, J. (2002). Crystal structures of the kinase domain of c-Abl in complex with the small molecule inhibitors PD173955 and imatinib (STI-571). Cancer Res 62, 4236-4243.

Nikolcheva, T., Pyronnet, S., Chou, S. Y., Sonenberg, N., Song, A., Clayberger, C., and Krensky, A. M. (2002). A translational rheostat for RFLAT-1 regulates RANTES expression in T lymphocytes. J Clin Invest 110, 119-126.

Nolen, B., Taylor, S., and Ghosh, G. (2004). Regulation of protein kinases; controlling activity through activation segment conformation. Mol Cell 15, 661-675.

Otwinowski, Z., and Minor, W. (1997). Processing of X-ray Diffraction Data Collected in Oscillation Mode. Meth Enz 276, 307-326.

Pargellis, C., Tong, L., Churchill, L., Cirillo, P. F., Gilmore, T., Graham, A. G., Grob, P. M., Hickey, E. R., Moss, N., Pav, S., and Regan, J. (2002). Inhibition of p38 MAP kinase by utilizing a novel allosteric binding site. Nat Struct Biol 9, 268-272.

Reiling, J. H., Doepfner, K. T., Hafen, E., and Stocker, H. (2005). Diet-dependent effects of the Drosophila Mnk1/Mnk2 homolog Lk6 on growth via eIF4E. Curr Biol 15, 24-30. 
Scheper, G. C., Morrice, N. A., Kleijn, M., and Proud, C. G. (2001). The mitogen-activated protein kinase signal-integrating kinase Mnk2 is a eukaryotic initiation factor $4 \mathrm{E}$ kinase with high levels of basal activity in mammalian cells. Mol Cell Biol 21, 743754.

Scheper, G. C., and Proud, C. G. (2002). Does phosphorylation of the cap-binding protein eIF4E play a role in translation initiation? Eur J Biochem 269, 5350-5359.

Taylor, S. S., and Radzio-Andzelm, E. (1994). Three protein kinase structures define a common motif. Structure 2, 345-355.

Tereshko, V., Teplova, M., Brunzelle, J., Watterson, D. M., and Egli, M. (2001). Crystal structures of the catalytic domain of human protein kinase associated with apoptosis and tumor suppression. Nat Struct Biol 8, 899-907.

Ueda, T., Watanabe-Fukunaga, R., Fukuyama, H., Nagata, S., and Fukunaga, R. (2004). Mnk2 and Mnk1 are essential for constitutive and inducible phosphorylation of eukaryotic initiation factor 4E but not for cell growth or development. Mol Cell Biol 24, 65396549.

Vieth, M., Sutherland, J. J., Robertson, D. H., and Campbell, R. M. (2005). Kinomics: characterizing the therapeutically validated kinase space. Drug Discov Today 10, 839846.

Walsh, D., and Mohr, I. (2004). Phosphorylation of eIF4E by Mnk-1 enhances HSV-1 translation and replication in quiescent cells. Genes Dev 18, 660-672.

Waskiewicz, A. J., Flynn, A., Proud, C. G., and Cooper, J. A. (1997). Mitogen-activated protein kinases activate the serine/threonine kinases Mnk1 and Mnk2. Embo J 16, 1909-1920.

Waskiewicz, A. J., Johnson, J. C., Penn, B., Mahalingam, M., Kimball, S. R., and Cooper, J. A. (1999). Phosphorylation of the cap-binding protein eukaryotic translation initiation factor 4E by protein kinase Mnk1 in vivo. Mol Cell Biol 19, 1871-1880.

Werten, S., Mitschler, A., Romier, C., Gangloff, Y. G., Thuault, S., Davidson, I., and Moras, D. (2002). Crystal structure of a subcomplex of human transcription factor TFIID formed by TATA binding protein-associated factors hTAF4 (hTAF(II)135) and hTAF12 (hTAF(II)20). J Biol Chem 277, 45502-45509. 
Yang, J., Cron, P., Good, V. M., Thompson, V., Hemmings, B. A., and Barford, D. (2002). Crystal structure of an activated Akt/protein kinase B ternary complex with GSK3peptide and AMP-PNP. Nat Struct Biol 9, 940-944. 


\section{Tables}

Table 1. Data collection and refinement statistics

\begin{tabular}{|c|c|}
\hline & Mnk1-KR wildtype \\
\hline \multicolumn{2}{|l|}{ Data collection } \\
\hline Space group & $\mathrm{P} 4_{3} 2{ }_{1} 2$ \\
\hline \multicolumn{2}{|l|}{ Cell dimensions } \\
\hline$a, b, c(\AA)$ & $93.5,93.5,175.2$ \\
\hline$\alpha, \beta, \gamma\left({ }^{\circ}\right)$ & $90,90,90$ \\
\hline Resolution $(\AA)$ & $30.0-2.8$ \\
\hline$R_{\text {sym }}$ or $R_{\text {merge }}$ & $10.3(34.1)$ \\
\hline$I / \sigma I$ & $9.9(1.9)$ \\
\hline Completeness (\%) & $89.8(43.0)$ \\
\hline \multicolumn{2}{|l|}{ Refinement } \\
\hline Resolution ( $\AA$ ) & $30.0-2.8$ \\
\hline No. reflections & 17771 \\
\hline$R_{\text {work }} / R_{\text {free }}$ & $23.0 / 28.3$ \\
\hline \multicolumn{2}{|l|}{ No. atoms } \\
\hline Protein & 2905 \\
\hline Water & 19 \\
\hline \multicolumn{2}{|l|}{$B$-factors } \\
\hline \multicolumn{2}{|l|}{ Protein } \\
\hline Chain A & 38.12 \\
\hline Chain B & 60.17 \\
\hline Waters & 28.1 \\
\hline \multicolumn{2}{|l|}{ R.m.s deviations } \\
\hline Bond lengths $(\AA)$ & 0.007 \\
\hline Bond angles $\left({ }^{\circ}\right)$ & 1.1 \\
\hline
\end{tabular}




\section{Supplementary Chapter III: Structures of Escherichia coli NAD Synthetase with substrates and products reveal mechanistic rearrangements}

\section{Citation:}

Jauch, R., Humm, A., Huber, R., and Wahl, M. C. (2005). Structures of Escherichia coli NAD synthetase with substrates and products reveal mechanistic rearrangements. J Biol Chem 280, 15131-15140.

\section{Statement of Contribution:}

structure refinement, model building, structure analysis and structural comparison, manuscript preparation 


\title{
Structures of Escherichia coli NAD Synthetase with Substrates and Products Reveal Mechanistic Rearrangements*
}

Received for publication, November 22, 2004, and in revised form, January 26, 2005 Published, JBC Papers in Press, February 7, 2005, DOI 10.1074/jbc.M413195200

\author{
Ralf Jauch $\$$, Andreas Humm§, Robert Huberף, and Markus C. Wahl|*** \\ From the $\$$ Max-Planck-Institut für Biophysikalische Chemie, Abteilung Molekulare Entwicklungsbiologie, \\ Am Fassberg 11, D-37077 Göttingen, Germany, §Roche Diagnostics GmbH, Nonnenwald 2, 82372 Penzberg, Germany, \\ १Max-Planck-Institut für Biochemie, Strukturforschung, Am Klopferspitz 18a, D-82152 Martinsried, Germany, and \\ |Max-Planck-Institut für Biophysikalische Chemie, Abteilung Zelluläre Biochemie/Röntgenkristallographie, \\ Am Fassberg 11, D-37077 Göttingen, Germany
}

\begin{abstract}
Nicotinamide adenine dinucleotide synthetases (NADS) catalyze the amidation of nicotinic acid adenine dinucleotide (NAAD) to yield the enzyme cofactor nicotinamide adenine dinucleotide (NAD). Here we describe the crystal structures of the ammonia-dependent homodimeric NADS from Escherichia coli alone and in complex with natural substrates and with the reaction product NAD. The structures disclosed two NAAD/NAD binding sites at the dimer interface and an adenosine triphosphate (ATP) binding site within each subunit. Comparison with the Bacillus subtilis NADS showed pronounced chemical differences in the NAAD/NAD binding sites and less prominent differences in the ATP binding pockets. In addition, the $E$. coli NADS structures revealed unexpected dynamical rearrangements in the NAAD/NAD binding pocket upon NAAD-to-NAD conversion, which define a catalysis state and a substrate/product exchange state. The two states are adopted by concerted movement of the nicotinysyl moieties of NAAD and NAD, Phe-170, and residues 224-228, which may be triggered by differential coordination of a magnesium ion to NAAD and NAD. Phylogenetic structure comparisons suggest that the present results are relevant for designing species-specific antibiotics.
\end{abstract}

Nicotinamide adenine dinucleotide $(\mathrm{NAD})^{1}$ is a ubiquitous carrier of reduction equivalents and functions as a cofactor in numerous metabolic reactions. NAD plays further roles in processes as diverse as calcium mobilization, DNA repair, and post-translational modification of proteins in eukaryotes. The final step of NAD biosynthesis is catalyzed by the enzyme NAD synthetase (NADS) and involves the conversion of nicotinic acid adenine dinucleotide (NAAD) to NAD (1) (see Fig. 1A). NAD synthetase activity was first described in the late $1950 \mathrm{~s}$ $(2,3)$. Two types of NADS enzymes have been discovered; the first using preferentially ammonia as the amide donor, the second employing glutamine instead. The corresponding reaction schemes have been reported in 1967 (4).

$$
\begin{gathered}
\mathrm{ATP}+\mathrm{NAAD}+\mathrm{NH}_{3} \rightarrow \mathrm{AMP}+\mathrm{PP}_{\mathrm{i}}+\mathrm{NAD} \\
\text { REACTION } 1
\end{gathered}
$$

$\mathrm{ATP}+\mathrm{NAAD}+$ L-glutamine $\rightarrow \mathrm{AMP}+\mathrm{PP}_{\mathrm{i}}+\mathrm{NAD}+$ L-glutamate

REACTION 2

In the course of the above reactions, the enzymes mediate an adenylyl transfer from ATP to the nicotinysyl moiety of NAAD, resulting in NAD-adenylate (see Fig. $1 B$ ) as a reaction intermediate. The second step of the reaction is the amidation of the nicotinysyl moiety using either ammonia (Reaction 1) or glutamine (Reaction 2) as an amide source.

NADSs from several prokaryotes, including Bacillus subitilis (bsu) (5), Escherichia coli (eco) (6, 7), and Mycobacterium tuberculosis ( $m t u$ ) (8) have been cloned and characterized. Both ecoNADS and $b s u$ NADS exhibit a clear preference for ammonia over glutamine $(2,5,7)$, whereas the mtuNADS can use both glutamine and ammonia as amide donors (8). Recently, the human (NADsyn1, here referred to as NADS1) and yeast (Qns1) NADSs have been characterized (9-11). Eukaryotic NADSs confer an additional N-terminal domain with nitrilase activity, which enables them to use glutamine as an amide donor (10).

NADS from Bacillus subtilis has been subjected to intensive crystallographic investigations. At first, the crystal structures of the bsuNADS apoenzyme, the complex of bsuNADS with ATP, and the complex of bsuNADS with the reaction intermediate NAD-adenylate had been reported (12-14). Subsequently, the picture was expanded by co-crystallizing bsuNADS with NAAD, AMP $+\mathrm{PP}_{\mathrm{i}}$ (a combination of NAAD and ATP), and also with the ATP analog AMP-CPP (15). More recently, a $1.0-\AA$ resolution structure of $b s u$ NADS in complex with the NAD-adenylate intermediate has been described (16). The combined structural studies unraveled the overall architecture of a NADS and delineated its mode of substrate binding. They defined separate binding sites for the ATP co-substrate (ATP binding pocket) and the substrate NAAD (NAAD/ NAD binding pocket), and they revealed some details of the catalytic mechanism. NADS blocking agents have been suggested to be promising antibiotics for pathogens, such as $B a$ cillus anthracis, which may be developed employing the bsuNADS crystal structures $(17,18)$.

In the present study, we provide high resolution crystal structures of NADS from $E$. coli, which shed light on evolutionary relationships among the enzymes ecoNADS and $b s u$ NADS. 
In addition to the structure of the apoenzyme and complexes of the enzyme with $\mathrm{AMP}, \mathrm{NAAD}$, and $\mathrm{AMP}+\mathrm{PP}_{\mathrm{i}}$, we present also the structure of NADS in association with its reaction product, NAD. This structure exhibits some unexpected rearrangements both in the active site and in the product molecules upon conversion of NAAD to NAD. The results reveal novel dynamical aspects for the reaction mechanism for ammonia-dependent NADSs. This work should also aid in the design of speciesspecific inhibitors for this class of enzymes.

\section{EXPERIMENTAL PROCEDURES}

Cloning, Expression and Purification-The E. coli nadE gene encoding NADS was PCR-amplified with $E$. coli $\mathrm{DH} 5 \alpha$ cells as the template source and was inserted into the EcoRI site of the expression plasmid pRSETHE (derived from pRSET6d (19)). The expression product was designed to contain an N-terminal hexahistidine tag (tag sequence MAHHHHHHEF). The DNA construct was verified by automated sequencing, which predicted a protein product in accordance with SwissProt accession number P18843.

The protein was expressed in shaking cultures of $E$. coli BL21(DE3) cells at $32{ }^{\circ} \mathrm{C}$ overnight by the addition of $1 \mathrm{~mm}$ isopropyl- $\beta$-D-thiogalactopyranoside during the mid-log phase of growth. The cells were harvested by centrifugation, resuspended in buffer A ( $50 \mathrm{~mm}$ Tris- $\mathrm{HCl}$, $\mathrm{pH}$ 7.0, $100 \mathrm{~mm} \mathrm{NaCl}, 0.2 \mathrm{~mm}$ phenylmethylsulfonyl fluoride) and lysed by sonification (Branson sonifier, Schwäbisch Gmünd, Germany). The His-tagged protein was captured on a nickel-nitrilotriacetic acid column (Qiagen, Düsseldorf, Germany) in buffer A, washed with buffer B (50 $\mathrm{mm}$ Hepes- $\mathrm{NaOH}, \mathrm{pH} 7.0,100 \mathrm{~mm} \mathrm{NaCl}, 50 \mathrm{~mm}$ imidazole, $0.2 \mathrm{~mm}$ phenylmethylsulfonyl fluoride), and eluted in a linear gradient to buffer C (50 mu Hepes- $\mathrm{NaOH}, \mathrm{pH}$ 7.0, $100 \mathrm{~mm} \mathrm{NaCl}, 800 \mathrm{~mm}$ imidazole, 0.2 $\mathrm{mm}$ phenylmethylsulfonyl fluoride). The NADS-containing fractions were pooled, dialyzed against buffer $\mathrm{D}$ (30 mu Hepes- $\mathrm{NaOH}, \mathrm{pH}$ 8.0, 0.1 mM EDTA) and applied to a DEAE-cellulose column (DE-52, Whatman, Brentford, UK), washed with buffer E (20 mM Tris-HCl, $\mathrm{pH} 8.0)$, and eluted in a linear gradient to buffer $\mathrm{F}(20 \mathrm{~mm}$ Tris- $\mathrm{HCl}, \mathrm{pH} 8.0,400 \mathrm{~mm}$ $\mathrm{NaCl}$. NADS eluted in the first of two peaks. The enzyme fractions were pooled, dialyzed against crystallization buffer $(20 \mathrm{~mm}$ Tris- $\mathrm{HCl}$, $\mathrm{pH}$ 7.0, $10 \mathrm{~mm} \mathrm{NaCl}, 0.2 \mathrm{~mm}$ dithiothreitol, $0.2 \mathrm{~mm}$ EDTA), and concentrated by ultrafiltration $(10-\mathrm{kDa}$ molecular mass cutoff membrane; Amicon, Billerica, MA) to $\sim 19 \mathrm{mg} / \mathrm{ml}$.

Crystallization and Data Collection-E. coli NADS apoenzyme was crystallized in hanging drops by vapor equilibration at room temperature against 12.5-18.5\% polyethylene glycol 400, $50 \mathrm{mM} \mathrm{MgCl}_{2}, 100 \mathrm{mM}$ sodium acetate, $\mathrm{pH} 5.0-5.4$, with a drop volume ratio of $2: 1$ (protein solution:reservoir). The addition of $1 \mu \mathrm{l}$ of a suspension of lithium-metatantalate significantly increased the nucleation rate and quality of the crystals. Crystals appeared and grew within a period of 1-14 days. NADS-ligand complexes were obtained by co-crystallization, adding 1.5-2 mM AMP (form II), ATP (form III), NAAD (form IV), or NAD (form V) to the setup (Table I).

Diffraction data were recorded at room temperature with a Rigaku (Tokyo, Japan) rotating anode x-ray generator equipped with a MarResearch (Hamburg, Germany) image plate. For data collection, crystals were mounted in thin walled special glass capillaries (Charles Supper Company, Natick, MA) with mother liquor on one side. Data were processed using the HKL software package (20).

Structure Solution and Refinement-The $E$. coli NADS-NAD co-crystal structure was solved by molecular replacement with the AMoRe software program (21) using the B. subtilis NADS coordinates (Protein Data Bank (PDB) code 1NSY) as a search model. The molecular replacement solution was subjected to rigid body refinement and simulated annealing using crystallography and NMR system (CNS) software package (22). The model was further improved by alternating cycles of manual model building using the XtalView software program (23) and positional and temperature factor refinement steps (CNS). During refinement, clear difference density appeared for the cofactor, which was manually inserted (XtalView). During the last cycles of refinement, water molecules were built automatically (CNS) into spherical peaks of the $F_{o}-F_{c}$ difference electron density and verified by manual inspection. During all refinement steps, $5 \%$ of the reflections were set aside to monitor the free $R$-factor.

All other NADS structures were subsequently solved by molecular replacement using the $E$. coli NADS coordinates as a search model and refined as outlined above. The final geometries of the models were evaluated using the PROCHECK program (24). For structure analyses, various programs from the Uppsala software factory (x-ray.bmc.uu.se/ usf/) and from the Collaborative Computational Project Number 4 (CCP4) program package (25) were employed. Coordinates and structure factors have been submitted to the Protein Data Bank (PDB codes 1WXE, 1WXF, 1WXG, 1WXH, 1WXI) and will be released upon publication.

\section{RESULTS}

Structure Solution and Quality of the Models-Recombinant ecoNADS was produced, purified, and crystallized as described under "Experimental Procedures." The crystal structures of the ecoNADS apoenzyme (form I; Table I) as well as four co-crystal structures with natural substrates and catalytic products (AMP, form II; AMP $+\mathrm{PP}_{\mathrm{i}}$, form III; NAAD, form IV; and NAD, form V; Table I) were solved by Patterson rotation/translation searches, using the structure coordinates of bsuNADS (PDB code 1NSY). The crystals of the enzyme complexes were obtained by co-crystallization and pertained to two different crystal systems (Table I). Employing diffraction data to maximum resolutions between 1.7 and $2.3 \AA$ without intensity cutoffs, the models were refined to $R_{\text {work }}$ and $R_{\text {free }}$ values between 17.1 and $22.3 \%$, maintaining good stereochemistry (Table I). All crystals comprised the full-length protein (residues 2-275), excluding the first methionine. No residues for the N-terminal hexahistidine affinity tag could be located in the electron densities. The short flexible loop P1 (residues 88-90) was not visible in the structures in which the ATP binding site is empty (forms I, IV, and V). A large loop P2 (residues 208-224; Fig. 2) could not be traced in either of the five crystal structures. Arg-186, located in a loop between $\beta 5$ and $\alpha 8$ (see below for a description of the topology), exhibited a disallowed dihedral angle conformation in all five models, which was maintained because of unambiguous $2 F_{o}-F_{c}$ electron density for this residue. The unusual conformation is stabilized through hydrogen bonds and ionic interactions with Glu-22, Ser-26, Pro-183, and Leu-184.

Global Structure and Comparison to the B. subtilis EnzymeecoNADS exhibits an $\alpha / \beta$ fold similar to the $B$. subtilis enzyme (Figs. 3 and $4 A$ ). $55 \%$ of all residues are found in $\alpha$-helical conformation, $15 \%$ belonging to $\beta$-structures. A twisted fivestranded parallel $\beta$-sheet is sandwiched between the two large helices $\alpha 3$ and $\alpha 6$. All $\beta$-strands are separated by single $\alpha$-helices, with the exception of $\beta 3$ and $\beta 4$, which are disrupted by two $\alpha$-helices ( $\alpha 5$ and $\alpha 6$ ). Thus, the core of ecoNADS comprises a typical Rossmann fold nucleotide binding motif.

In both space groups, $\mathrm{C} 2$ and $\mathrm{P} 3_{1} 21$, ecoNADS or its complexes crystallized with one protein molecule per crystallographic asymmetric unit. It has been well established that bacterial NADSs form homodimers $(7,12)$. In the present crystals, ecoNADS monomers are positioned close to crystallographic two-fold axes, which generate the functional dimers. The globular NADS homodimer has approximate dimensions of $77 \times 60 \times 56 \AA$. Based on an analysis with the protein-protein interaction server (26), $\sim 2500 \AA^{2}$ of combined surface area are buried in the dimer interface, encompassing $18 \%$ of the solventexposed surface area of the molecules. Contacts between the protomers are principally mediated by the $\alpha 5-\alpha 6$ region. The two pairs of helices align homotypically in an antiparallel fashion (Fig. 3). The dimer interface is predominantly nonpolar (75\% of the participating residues) and involves 14 hydrogen bonds but lacks intervening water molecules. Therefore, ecoNADS qualifies as an obligatory dimer.

$e c o N A D S$ and $b s u$ NADS are 55\% identical in their amino acid sequences (Fig. 2). Consistently, they exhibit quite similar overall folds. Fig. $4 A$ shows an overlay of the NAAD-containing models from both organisms, which exhibit an average root mean square deviation of $0.55 \AA$ for 241 aligned C- $\alpha$ atoms. Five regions in the $E$. coli protein contain more than one residue deviating by $>1 \AA$ from the $B$. subtilis structure (Fig. $4 A$ ): region 1 (residues 37-42) (E. coli numbering), region 2 
TABLE I

Crystallographic data

Data in parentheses for the last $0.05 \AA$ (for form I last $0.1 \AA$ ).

\begin{tabular}{|c|c|c|c|c|c|}
\hline \multicolumn{6}{|l|}{ Crystals } \\
\hline Form & I & II & III & IV & $\mathrm{V}$ \\
\hline Cofactor & $-^{c}$ & AMP & $\mathrm{AMP}+\mathrm{PP}_{\mathrm{i}}$ & NAAD & NAD \\
\hline Space group & $\mathrm{P} 3_{1} 21$ & $\mathrm{C} 2$ & $\mathrm{C} 2$ & $\mathrm{C} 2$ & $\mathrm{P} 3_{1} 21$ \\
\hline \multicolumn{6}{|l|}{ Unit cell parameters } \\
\hline a $(\AA)$ & 91.9 & 92.5 & 92.4 & 92.4 & 92.2 \\
\hline $\mathrm{b}(\AA)$ & 91.9 & 67.8 & 67.9 & 67.8 & 92.2 \\
\hline c $(\AA)$ & 76.3 & 48.3 & 48.4 & 48.2 & 76.2 \\
\hline$\alpha\left(^{\circ}\right)$ & 90 & 90 & 90 & 90 & 90 \\
\hline$\beta\left(^{\circ}\right)$ & 90 & 104.5 & 104.2 & 104.4 & 90 \\
\hline$\gamma\left({ }^{\circ}\right)$ & 120 & 90 & 90 & 90 & 120 \\
\hline PDB ID code & $1 \mathrm{WXF}$ & $1 \mathrm{WXE}$ & 1WXI & $1 \mathrm{WXG}$ & $1 \mathrm{WXH}$ \\
\hline \multicolumn{6}{|l|}{ Data collection } \\
\hline Resolution $(\AA)$ & $30.0-2.3$ & $30.0-1.8$ & $30.0-1.7$ & $30.0-1.9$ & $30.0-1.9$ \\
\hline Unique reflections & 16,081 & 25,734 & 29,229 & 21,526 & 29,775 \\
\hline Redundancy & 5.4 & 3.7 & 3.8 & 3.6 & 3.8 \\
\hline Completeness (\%) & $95.1(97.7)$ & $95.9(92.1)$ & $91.6(87.6)$ & 94.5 (91.7) & $99.5(95.3)$ \\
\hline$I / \sigma(I)$ & $14.4(3.0)$ & $13.0(2.9)$ & $14.7(2.7)$ & $11.9(2.3)$ & $19.6(3.0)$ \\
\hline$R_{\mathrm{sym}}{ }^{a}(\%)$ & $8.8(45.9)$ & $10.8(41.8)$ & $7.4(42.8)$ & $11.7(58.6)$ & $5.4(36.7)$ \\
\hline \multicolumn{6}{|l|}{ Refinement } \\
\hline Resolution & $30.0-2.3$ & $30.0-1.8$ & $30.0-1.7$ & $30.0-1.9$ & $30.0-1.9$ \\
\hline Reflections used & 16080 & 25734 & 29229 & 21526 & 29758 \\
\hline Protein molecules/asymmetric unit & 1 & 1 & 1 & 1 & 1 \\
\hline Protein atoms & 2008 & 2029 & 2051 & 2008 & 2008 \\
\hline Solvent atoms & 165 & 257 & 277 & 227 & 302 \\
\hline Nucleotide atoms & - & 46 & 55 & 44 & 44 \\
\hline $\mathrm{Mg}^{2+}$ & - & 1 & 2 & 1 & 1 \\
\hline$R_{\mathrm{work}} / R_{\text {free }}^{b}(\%)$ & $18.3 / 21.7$ & $19.1 / 21.1$ & $17.6 / 20.5$ & $19.9 / 22.3$ & $17.1 / 19.1$ \\
\hline \multicolumn{6}{|l|}{ Average temperature factors $\left(\AA^{2}\right)$} \\
\hline Protein & 36.4 & 18.7 & 18.9 & 20.3 & 28.2 \\
\hline Water oxygens & 47.8 & 33.9 & 38.1 & 34.2 & 50.4 \\
\hline Nucleotide (NAD/ATP sites) & $-1-$ & $19.5 / 45.1$ & $17.1 / 38.6$ & $29.0 /-$ & $38.3 /-$ \\
\hline $\mathrm{Mg}^{2+}$ & - & 42.9 & 59.2 & 33.5 & - \\
\hline Wilson $B$-factor & 38.9 & 26.5 & 26.8 & 24.5 & 33.3 \\
\hline \multicolumn{6}{|c|}{ Root mean square deviation from ideal values } \\
\hline Bond length $(\AA)$ & 0.005 & 0.005 & 0.005 & 0.005 & 0.005 \\
\hline Bond angle $\left({ }^{\circ}\right)$ & 1.08 & 1.20 & 1.10 & 1.13 & 1.15 \\
\hline \multicolumn{6}{|l|}{ Ramachandran analysis } \\
\hline Preferred $(\%)$ & 93.6 & 93.9 & 93.9 & 94.5 & 92.3 \\
\hline Additionally allowed (\%) & 6.0 & 5.7 & 5.3 & 5.0 & 7.3 \\
\hline Disallowed $(\%)$ & 0.4 & 0.4 & 0.8 & 0.5 & 0.4 \\
\hline
\end{tabular}

(residues 71-76), region 3 (residues 125-129), the far C terminus, and the C-terminal continuation of the P2 loop. Regions 1 and 2 exhibit single or double amino acid insertions in ecoNADS. Significantly, region 1 is intimately involved in binding of the adenylyl moiety in the NAAD/NAD binding pocket (see below). In contrast, the deviating regions 2,3 , and the far $\mathrm{C}$ terminus are distant from the substrate binding sites and are therefore not expected to exhibit effects on the substrate or product binding characteristics of the enzymes. The P2 loop has a fundamental impact on the catalytic mechanism (see "Architecture of the NAAD/NAD Binding Site").

Design of the ATP Binding Site-In the present ensemble of crystal structures, the ATP binding sites of form II and III crystals contained $\mathrm{AMP}$ and $\mathrm{AMP}+\mathrm{PP}_{\mathrm{i}}$, respectively, whereas the site was empty in the apoenzyme (form I), in the NAAD complex (form IV), and in the NAD complex (form V). Crystal form III was obtained by the addition of ATP to the crystallization drops, but the crystals contained AMP and pyrophosphate, indicating that hydrolysis of ATP took place during crystallization.

Each of the two ATP binding sites in the ecoNADS dimer is formed exclusively from one subunit. In crystal forms II and III, the ribose portions of the adenine nucleotides are engaged in hydrogen bonding interactions with the backbone oxygen of Gly-46 (to ribose O-2') and the side chain hydroxyl group of Thr-160 (to ribose O- $3^{\prime}$ ). The AMP adenine ring is held in place through stacking interactions with Arg-142, Ile-47, and Ser-48 (Fig. 5A). A hydrogen bond between $\mathrm{N}-6$ of the adenine moiety and Gln-88 leads to a stabilization of loop P1, as indicated by clear $2 F_{o}-F_{c}$ electron density for this protein region. In contrast, no electron density developed for loop P1 in the apoform or in other crystals with unoccupied ATP binding sites (forms I, $\mathrm{IV}$, and V). Flexibility in loop P1 is the only recognizable mechanism to yield access to the ATP binding site. Thus, the P1 loop serves as a lid, which is flexibly designed to allow ATP access but closes the ATP pocket upon substrate binding.

The side chain of Arg-82 exists in two conformations in the AMP co-crystal structures. In the major conformation, it interacts either with Gln-88 of the P1 loop and with $\mathrm{N}-1$ of the adenine moiety. In the minor conformation, it interacts with the side chains of Glu-91 and Gln-95 from helix $\alpha 4$ (Fig. 5A). The latter conformation of Arg-82 is the only form seen in crystal structures with unoccupied ATP binding sites (forms I, IV, and V). The conformation of Arg-82, in which it interacts with Gln-88 and the adenine of the nucleotide, thus seems to be induced upon ATP/AMP binding. Because the other conformation of Arg-82, in which it interacts with helix $\alpha 4$ residues and which is characteristic of the nucleotide-free state, is seen as a minor conformation in the $\mathrm{AMP}$ and $\mathrm{AMP}+\mathrm{PP}_{\mathrm{i}}$ co-crystal structures; a small portion of the protein molecules in these latter crystals presumably have no nucleotide bound. Taken together, Arg-82 in ecoNADS seems to play the role of a sub- 
FIG. 1. Chemical structures. $A$, structure of the reaction substrate $(\mathrm{NAAD})$ and product (NAD). $B$, structure of the reaction intermediate NAD-adenylate.

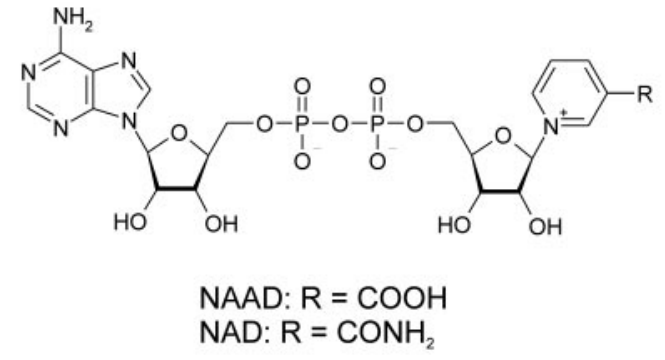

$B$ strate sensor or gate keeper for the ATP binding pocket.

As in most ATP-binding proteins, several magnesium ion binding sites have been reported for bsuNADS (14). In the present ecoNADS structures, a $\mathrm{Mg}^{2+}$ binding site (site I) is clearly occupied as well in crystal forms II (AMP), III $\left(\mathrm{AMP}+\mathrm{PP}_{\mathrm{i}}\right)$, and IV $(\mathrm{NAAD})$, i.e. in structures that contain at least AMP at the ATP binding site or NAAD at the NAAD/NAD binding site. The $\mathrm{Mg}^{2+}$-I ions show a typical octahedral coordination sphere, which in the $\mathrm{AMP}+\mathrm{PP}_{\mathrm{i}}$ co-crystal structure (form III) is composed of oxygens from the AMP $\alpha$-phosphate, the two phosphate groups of the pyrophosphate moiety (former $\beta$ - and $\gamma$-phosphates of ATP), the side chain carboxyls of Asp-51 and Glu-165 and a water molecule (Fig. 5C). In the AMP co-crystal structure (form II), which lacks the pyrophosphate molecule, and in the NAAD co-crystal structure (form IV), in which the ATP binding pocket is unoccupied, missing phosphate oxygens are replaced by water molecules in the coordination sphere.

In crystals of form IV (NAAD co-crystal), the nicotinysyl portion of NAAD is oriented toward $\mathrm{Mg}^{2+}-\mathrm{I}$. The nicotinysyl carboxyl group of NAAD is engaged in a hydrogen bond with a water oxygen, which belongs to the octahedral coordination sphere of $\mathrm{Mg}^{2+}-\mathrm{I}$ (Fig. 5C). The latter water oxygen coincides with the position of an $\alpha$-phosphate oxygen in forms II (AMP) and III $\left(\mathrm{AMP}+\mathrm{PP}_{\mathrm{i}}\right)$ structures. In contrast, the form $\mathrm{V}$ structure (NAD co-crystal) does not contain the $\mathrm{Mg}^{2+}$-I ion. Correlating with this observation, the nicotinysyl moiety of NAD is differently oriented from the corresponding portion of NAAD and is pointing away from the $\mathrm{Mg}^{2+}$-I binding site (see "Architecture of the NAAD/NAD Binding Site"). Therefore, in the NAAD co-crystal structure, $\mathrm{Mg}^{2+}-\mathrm{I}$ is seen as a direct link between the ATP binding pocket and the NAAD/NAD binding pocket. This direct interaction is lost upon formation of the product NAD.

A second magnesium ion $\left(\mathrm{Mg}^{2+}-\mathrm{II}\right)$ has been reported in bsuNADS crystal structures $(14,15)$, where it is coordinated by the AMP $\alpha$-phosphate, two pyrophosphate oxygens, two water molecules, and the backbone carbonyl oxygen of Thr-208 (B. subtilis numbering). In the present ensemble of structures, the $\mathrm{Mg}^{2+}-\mathrm{II}$ ion is only seen in form III crystals $\left(\mathrm{AMP}+\mathrm{PP}_{\mathrm{i}}\right.$ complex). Because $\mathrm{Mg}^{2+}-\mathrm{II}$ is present only in the $\mathrm{AMP}+\mathrm{PP}_{\mathrm{i}}$ complex, the pyrophosphate (or by inference the $\beta$ - and $\gamma$-phosphate groups of ATP) is required to capture a magnesium ion at site II in ecoNADS.

Comparison of the ATP Binding Sites of E. coli and B. subtilis NADS-Ordering of the P1 loop upon occupation of the ATP binding site has also been seen in bsuNADS. Therefore, loop P1 seems to be a conserved flexible element, the function of which is to close the ATP binding site upon the addition of a nucleotide. In ecoNADS, we have observed an additional conformational change in the side chain of Arg-82 upon AMP $+\mathrm{PP}_{\mathrm{i}}$ binding, which is consistent with Arg-82 constituting a sensor for the occupation of the ATP pocket (Fig. 5A). In contrast, according to available models for the $B$. subtilis protein, the Arg-82 conformation of the nucleotide-bound forms is preformed in the bsuNADS apoenzyme. Thus, despite a high degree of similarity in the chemical composition of the ATP binding pockets, ecoNADS and bsuNADS seem to respond differently to nucleotide binding.

Significantly, in bsuNADS, the N-terminal part of loop P2 contributes the backbone carbonyl oxygen of Thr-208 (B. subtilis numbering) for coordination of $\mathrm{Mg}^{2+}-\mathrm{II}$ in the ATP binding pocket. Loop P2 is ordered in structures of the Bacillus enzyme, in which nucleotides occupy the ATP pocket, portraying how this region can fold back onto the bulk of the protein (Fig. 4B). In contrast, loop P2 is disordered in the present structures of ecoNADS. The sequence of loop P2 is highly conserved between the two enzymes, and ecoNADS also comprises a threonine residue corresponding to Thr-208 $8^{\text {bsu }}$ (Thr-210 ${ }^{\text {eco }}$; superscripts identify the organism). However, although the $\mathrm{AMP}+\mathrm{PP}_{\mathrm{i}}$ cocrystal structure (form III) of ecoNADS contains a $\mathrm{Mg}^{2+}$-II, the backbone of the P2 loop remains disordered. It is possible that one ligand of the $\mathrm{Mg}^{2+}$-II coordination sphere in the ecoNADS form III structure, which has been modeled as a water molecule, actually corresponds to the backbone carbonyl of Thr$210^{\text {eco }}$ and that the P2 loop becomes partially ordered upon occupation of the $\mathrm{Mg}^{2+}$-II site. However, full ordering of the P2 loop seems to be supported by crystal packing contacts. In the 
B.subtilis B.anthracis E.coli H.sapiens S.cerevisiae

B.subtilis B. anthracis E.coli H. sapiens S.cerevisiae

B.subtilis B.anthracis E.coli

H. sapiens S.cerevisiae

B.subtilis

B. anthracis

E.coli

H.sapiens

S.cerevisiae
1

$1 \mathrm{MTLO}$

1 MTLQQQI IKALGAKPQIN 271 ATVDLEEVRSYRAAVMSRGLQASLAEIKFKRIDIPVELALMTSRFDPTVCPTKVREPFYH
B.subtilis B.anthracis E.coli H. sapiens S.cerevisiae
18 -PKQEIEDR-VNFLRQYVKKTG-AKGFVLGISGGQDSTLAGRLAQLAVESIREEGGD- - A 19 -PKAEIRKR-VDFLKDYVKKTG-AKGFVLGISGGQDSTLAGRLAQLAVEEIRNEGGN- - A 19 -AEEEIRRS -VDFLKSYLQTYPF IKSLVLGI SGGQDSTLAGKLCQMAINELRLETGNESL 327 SPEEEISLGPACWLWDFLRRSQ-QAGFLLPLSGGVDSAATACLIYSMCCQVCEAVRSGNE 331 SPEEEIALGPACWMWDYLRRCN-GTGFFLPLSGGIDSCATAMIVHSMCRLVTDAAQNGNE
73

74

\section{QFIAVR TFIAVR QFIAVR- -} EVL Q
*

\section{$\mathrm{OOO}$}

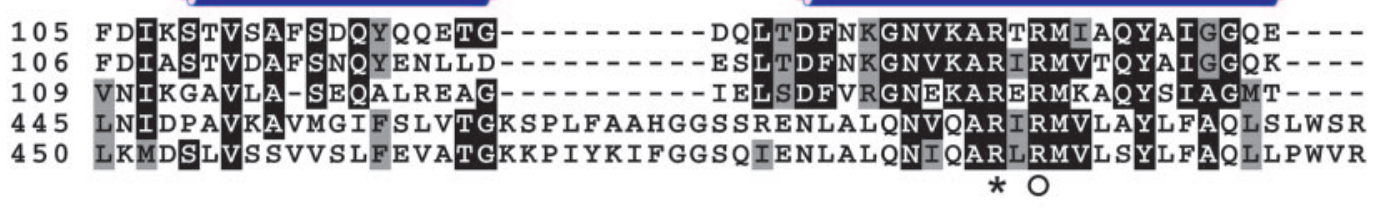

151 - - - - -GLLVLGTDHAAEAVTGFFTKYGDGGADLLPLTGLTKRQGRTLLK - - - - - ELGA 152 - - - - GLLVIGTDHAAEAVTGFFTKFGDGGADLLPLTGLTKRQGRALLQ - - - - - ELGA 154 -- - - SGVVVGTDHAAEAITGFFTKYGDGGTDINPLYRLNKRQGKQLLA - - - - ALAC 505 GVH--GGLLVLGSANVDESLLGYLTKYDCSSADINPIGGISKTDLRAFVQFCIQRFQLPA 510 GIPNSGGLLVLGSANVDECLRGYLTKYDCSSADINPIGGISKTDLKRFIAYASKQYNMPI
B.subtilis B. anthracis E.coli

H. sapiens

s.cerevisiae
199 PERLYLREPTADLL-- -DEKPQQSDETELGI SYDEIDDY -

200 DERLYLKMP TA DLL - - DEKP GQADETELGITYDQLLDDY

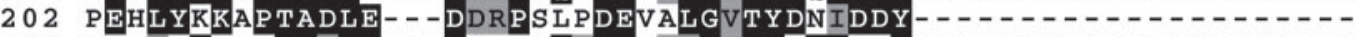
563 LQSILLAPATAELEPLADGQVSQTDEEDMGMTYAELSVYGKLRKVAKMGPYSMFCKLLGM 570 LNDFLNATPTAELEPMTKDYV-QSDEIDMGMTYEELGVFGYLRKVEKCGPYSMFLKLLHQ

\author{
B.subtilis \\ B. anthracis \\ E.coli \\ H. sapiens \\ S.cerevisiae
}

235 LEGKEVSAKVSEALEKRYSMTEHKRQVPASMF DDWWK- - - -

236 LEGKTVPADVAEKIEKRYTVSEHKRQVPASMF DDWWK238 LEGKNVPQQVARTIENWYLKTEHKRRP PITVF DDFWK623 RRHICTPRQVADKVKRFF SKYSMNRHKMTTLTPAYHAENYSPEDNRFDLRPFLYNTSWPW 629 WSPKLTPRQISEKVKRFFFFYAINRHKQTVLTPSYHAEQYSPEDNRF DLRPFLINPRFPW **

\section{B.subtilis \\ B. anthracis \\ E.coli \\ H. sapiens \\ S.cerevisiae \\ 689 ASRKIDEVVEQCEAHKGSTLDIMSID}

IIG. 2. Sequence alignment. Alignment of B. subtilis, B. anthracis, E. coli, human (NADsyn1) and Saccharomyces cerevisiae (Qnsp1) NADSs Human and yeast proteins comprise a number of insertions. An additional N-terminal domain in these proteins, not found in the bacterial NADSs, has been omitted from the alignment. Asterisks highlight residues involved in NAD/NAAD binding in bacteria. Closed circles indicate residues involved in the coordination of $\mathrm{Mg}^{2+}$-I, and open circles indicate residues, which interact with ATP at the ATP site. Blue helix icons above the alignment denote $\alpha$-helices, and red arrows denote $\beta$-strands as found in the ecoNADS structure. Green bars above the alignment denote loops P1 (residues 88-90 of ecoNADS) and P2 (residues 209-222) The alignment was generated using the ClustalW program (www.ebi.ac.uk/clustalw/).

bsuNADS crystal structures, residues Glu- $214^{b s u}$ and Lys$215^{\text {bsu }}$ from the central portion of loop P2 are involved in salt bridges to a neighboring molecule of the crystal lattice. In contrast, the P2 region in both ecoNADS crystal systems (C2 and $\mathrm{P} 3_{1} 21$ ) faces a large solvent channel, creating a solutionlike environment around this region of the enzyme. 
FIG. 3. Overall structure of ecoNADS. Upper panel, stereo ribbon plot of an $E$. coli NADS homodimer. One NADS molecule $(\mathrm{Mol} A)$ is color-coded by secondary structure ( $\alpha$-helices in blue, $\beta$-strands in red, and loops in gray). The other ( $\mathrm{Mol}$ $B$ ) is shown in black. Flexible loops P1 and $\mathrm{P} 2$ are shown in yellow. To indicate the respective binding pockets, NAD, $\mathrm{AMP}$, and $\mathrm{PP}_{\mathrm{i}}$ (as found in different crystal structures) are shown as stick figures. $\mathrm{Mg}^{2+}$ ions are indicated as spheres. Cofactors of molecule A are color-coded by atom type (carbon in gray, oxygen in red, nitrogen in blue, phosphorus in pink, and magnesium in green). Cofactors of molecule B are in brown. By default, structure figures were generated using the PyMOL program (pymol.sourceforge.net/). Lower panel, topology plot generated with Ligplot (27). $N, \mathrm{~N}$ terminus; $C, \mathrm{C}$ terminus.
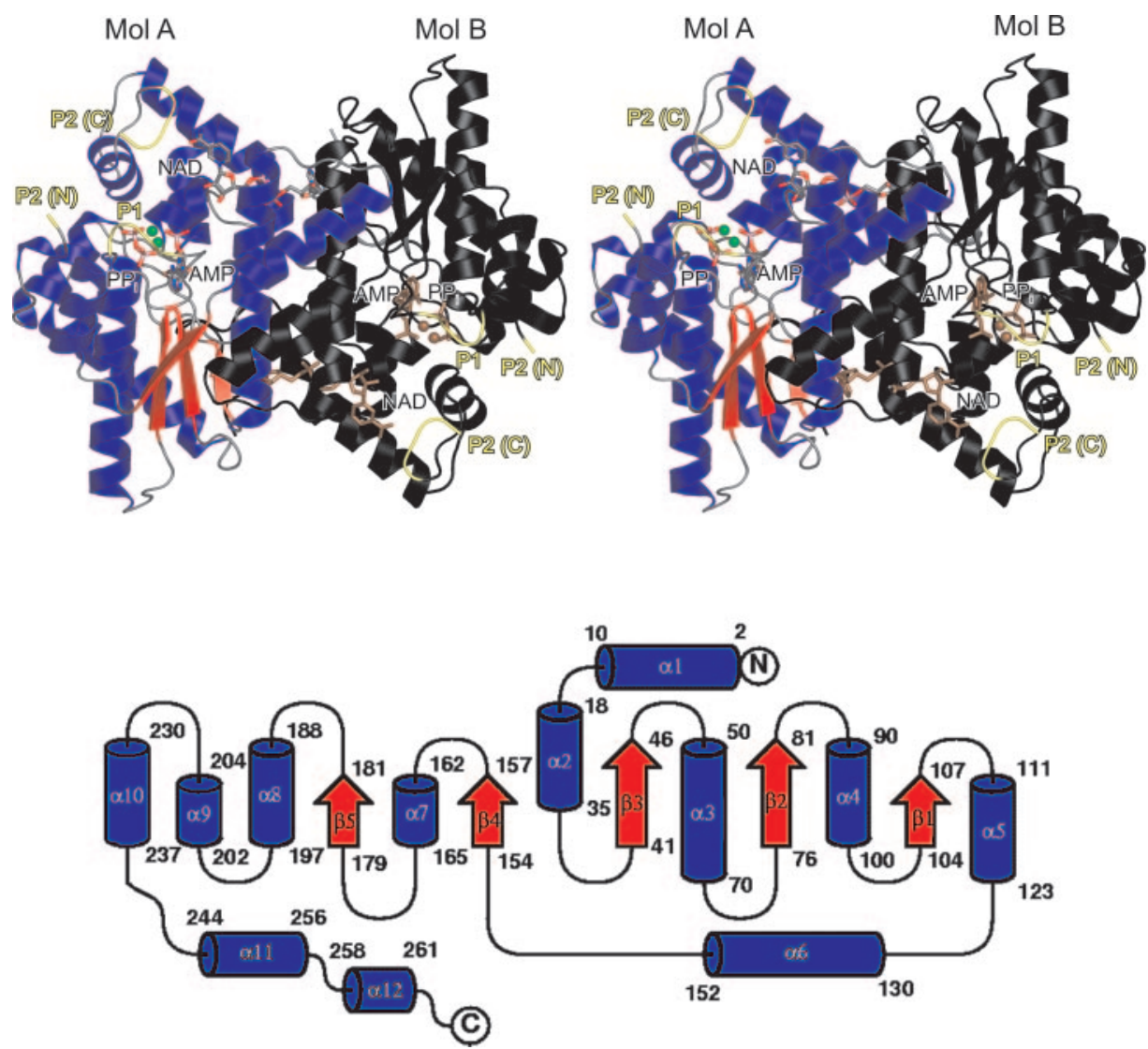

In conclusion, we note that the $\mathrm{N}$-terminal part of loop P2 can adopt various degrees of ordering, completely fixed (as in the structures of bsuNADS with nucleotides in the ATP pocket), partially ordered (as in the structure of $\mathrm{AMP}+\mathrm{PP}_{\mathrm{i}}$ of ecoNADS), and completely disordered (as in the structures of ecoNADS, in which $\beta$ - and $\gamma$-phosphates are lacking from the ATP binding site). To some degree, ordering of loop P2 thus seems to be dependent upon occupation of the $\mathrm{Mg}^{2+}$-II site, the fixation of which in turn depends on the presence and mode of binding of the ATP $\beta$ - and $\gamma$-phosphates or, after hydrolysis, the $\mathrm{PP}_{\mathrm{i}}$ leaving group. The interplay between the second $\mathrm{Mg}^{2+}$ ion and the N-terminal part of loop P2 therefore correlates with the state of the nucleotide in the ATP binding pocket.

Architecture of the NAAD/NAD Binding Site-The two identical NAAD/NAD binding sites per homodimer are located at the dimer interface (Fig. 3). Both subunits provide residues for NAAD/NAD binding (in the following termed subunit $\mathrm{A}$ and subunit B). In the present structures, we observed three different nucleotides at the NAAD/NAD binding site: AMP in forms II (AMP co-crystallization) and III (ATP co-crystallization), NAAD in form IV, and NAD in form V. Thus, co-crystallization in the presence of adenine nucleotides (but without NAD or NAAD) leads to the occupation of the NAAD/NAD binding site by AMP.

Binding of the adenosyl portions of the nucleotides by the enzyme is identical in the co-crystal structures with NAAD (form IV) and NAD (form V), and the adenosyl parts are tightly fixed at the dimer interface by these interactions. The adenine ring of the AMP portion of the nucleotides is sandwiched between His-260 from subunit A and Ile-40/Val-156 from subunit B. Tyr- $33^{\mathrm{B}}$ (superscripts identify the subunit) is engaged in a hydrogen bonding interaction with the adenine $\mathrm{N}-1$ atom. The adenylyl ribose interacts via its $3^{\prime}-\mathrm{OH}$ with Asp- $180^{\mathrm{B}}$ (carbonyl oxygen) and via O- $4^{\prime}$ with His- $260^{\mathrm{A}}$ (atom $\mathrm{N}^{\epsilon 2}$ ). The phosphate groups interact with the side chains of Lys-261 ${ }^{\mathrm{A}}, \mathrm{Arg}-140^{\mathrm{A}}$, and Asn- $136^{\mathrm{A}}$ and with the backbone nitrogen of Lys-173 ${ }^{\mathrm{A}}$.

In contrast, the nicotinysyl parts of both NAAD and NAD are fixed to a lesser extent compared with the adenosyl parts, indicated by higher temperature factors. However, the conformation of both NAAD and NAD could be unambiguously determined in $F_{o}-F_{c}$ "omit" electron density maps (Fig. $5 B$ ). The nicotinic acid ring of NAAD is stabilized by the side chains of Phe- $170^{\mathrm{A}}$, Thr- $172^{\mathrm{A}}$, and Asp- $176^{\mathrm{A}}$. The NAD conformation in form $\mathrm{V}$ crystals deviates significantly from the NAAD conformation (Fig. $3 C$ ). Relative to the NAAD structure, the nicotinamide moiety of NAD is rotated around the $\mathrm{O}-5^{\prime}-\mathrm{C}-5^{\prime}$ bond (Fig. 5C). Supporting the alternate NAD conformation, Phe170 undergoes a concomitant structural change, from stacking on the NAAD nicotinysyl ribose to stacking with the nicotinamide ring of NAD (Fig. $5 C$ ). The conformation of Phe-170 in the apoenzyme structure resembles its preferred conformation in the NAD co-crystal structure. In contrast, the structures of ecoNADS, in which AMP is bound to the NAD site (forms II and III), exhibit a NAAD-like Phe-170 conformation.

The conformation of loop P2 also appears to be subjected to rearrangements in the course of the enzymatic reaction. Although loop P2 could be traced neither in the form IV (NAAD) nor in the form V (NAD) structures as mentioned above, the conformation of the residues 224-228, which comprise the first traceable part after the disordered stretch 208-223, is strikingly different in the NAD complex compared with the NAAD co-crystal structure (Fig. $5 C$ ). The regions of the two structures exhibit C- $\alpha$ root mean square deviation values of $>3 \AA$. Resembling the behavior of Phe-170, the conformation of region 224228 in form V (NAD) recalls the conformation of the apoform (form I), whereas its conformation in form IV (NAAD) is highly similar to the models with occupied ATP sites (forms II and III). Significantly, the preferred conformation of NAD in the NAAD/ NAD pocket and the conformation of the surrounding enzyme 
FIG. 4. Phylogenetic comparison. $A$, stereo view of a superpositioning of the NAAD co-crystal structures of $E$. coli NADS (ecoNADS) (left subunit in light blue and right subunit in black) and B. subtilis NADS (bsuNADS) (both subunits in red; PDB code 1IFX). The NADS homodimers are shown in the same orientation as in Fig. 3. Cofactors are indicated by broad sticks (color-coded by atom type: oxygen in red, nitrogen in blue, and phosphorus in pink). Carbons for ligands of the left subunit are light blue, and those for ligands of the right subunit are gray). Besides NAAD, which was co-crystallized with the enzymes shown, AMP and $\mathrm{PP}_{i}$ from the form III crystal structure are superimposed to indicate the ATP binding pockets. Regions with a C- $\alpha$ root mean square deviation $>1 \AA$ between the two enzymes are colored in green in the left subunit of ecoNADS (region 1, residues $36-41$; region 2 , residues $70-75$; region 3 , residues 124-128). $B$, detailed stereo view of a similar superpositioning of ecoNADS (NAD complex) with bsuNADS (NAD-adenylate complex), indicating residues for the P2 loop in yellow (bsuNADS) and for residues neighboring the P2 loop in green (ecoNADS). Other coloring and the orientation of the figure are identical to those of the left subunit in $A$. C, comparison of region 1 from the two enzymes, which contains residues close to the NAAD/NAD binding site after global superpositioning of the molecules. Atoms are color-coded by type as above. Carbons and backbone traces of ecoNADS are shown in light blue, and those of bsuNADS are in red. Residues of ecoNADS are labeled in single letter style. The NAAD molecules superimpose almost perfectly; therefore, only one (from ecoNADS) is shown for clarity.
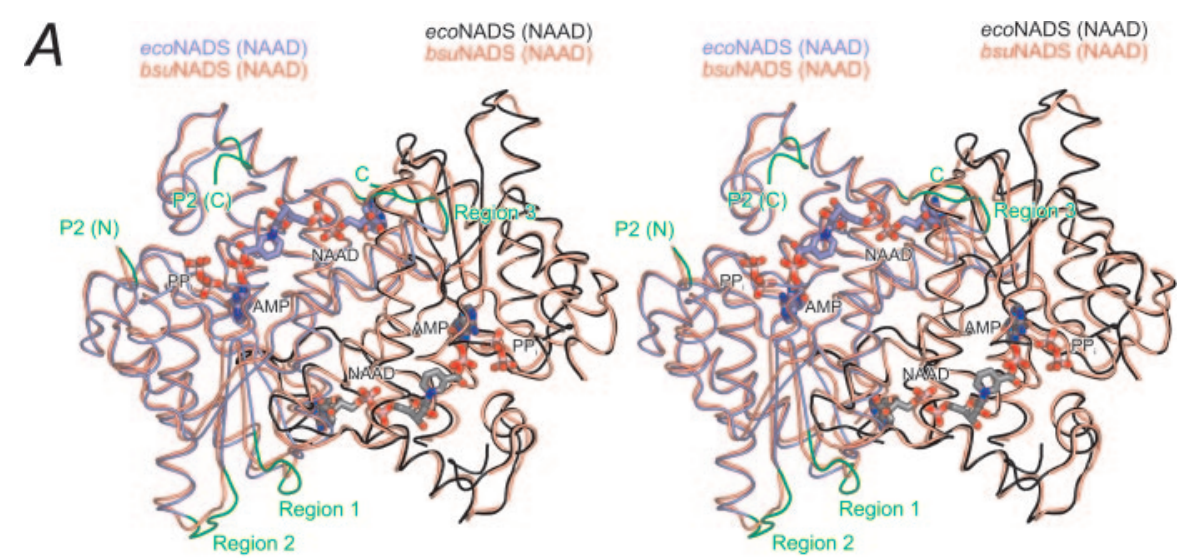

$B$
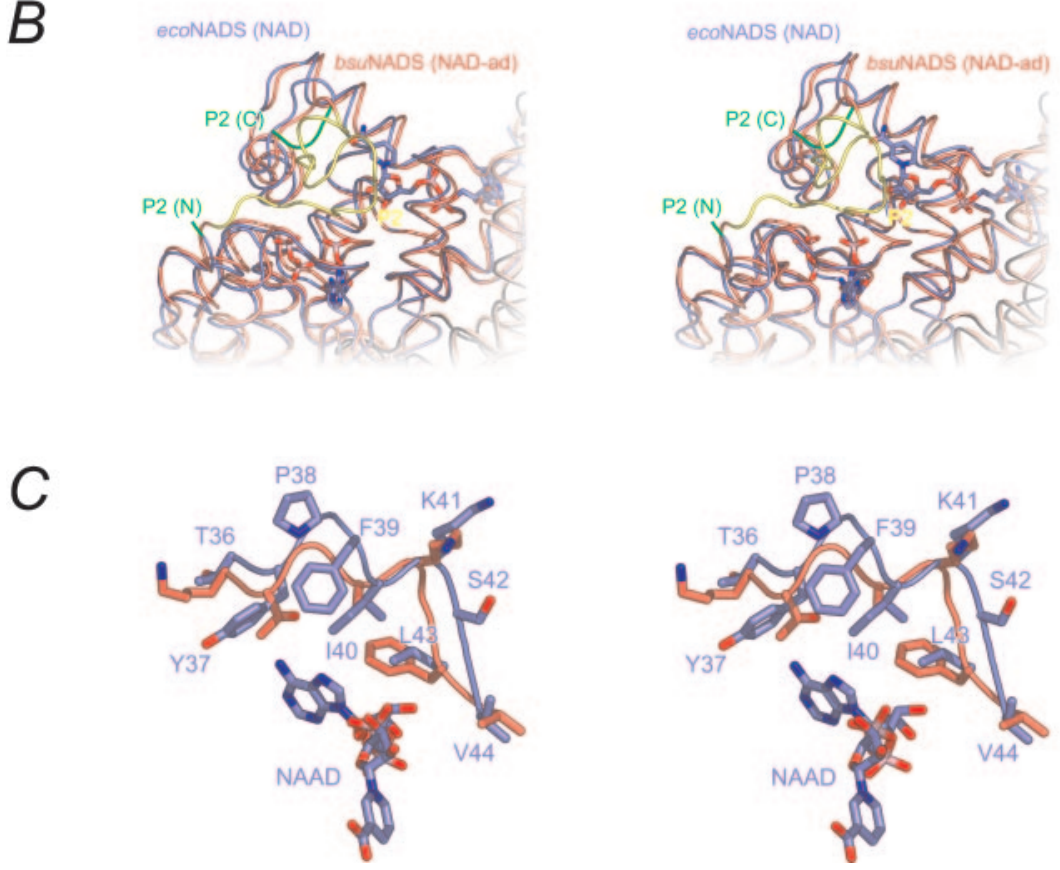

regions (Phe-170 and residues 224-228) in the NAAD co-crystal structure, and vice versa, are mutually exclusive. Superposition of the NAD-ecoNADS and NAAD-ecoNADS co-crystal structures clearly show a sterical hindrance for adoption of the NAAD-bound conformation of region 224-228 when NAD is present and vice versa (Fig. $5 C$ ). In this regard, it is important to note that the change in conformation of ecoNADS region 224-228 and of Phe-170 also correlates with the presence of $\mathrm{Mg}^{2+}$-I, which we have shown above to be involved in orientation of the substrate NAAD, but which is lacking when NAD is present. Therefore, release of $\mathrm{AMP}+\mathrm{PP}_{\mathrm{i}}$ and concomitant loss of $\mathrm{Mg}^{2+}$-I may be involved in the adoption of the NAD/apoform structures of the enzyme.

In conclusion, two alternative states of the NAAD/NAD binding site exist in ecoNADS, represented by alternative conformations observed for Phe-170, residues 224-228 (and most likely the remainder of the P2 loop), and the nicotinysyl moieties of NAAD and NAD, as well as the differential coordination of NAAD and NAD to $\mathrm{Mg}^{2+}$-I. One conformation, which is observed in the apoform and in the NAD-bound form of the enzyme and which we term the "exchange (E) state," seems to allow substrate binding and product release. The other conformation, which is formed upon occupation of the ATP binding site or in the presence of the substrate NAAD and which we term the "catalysis (C) state," has to be adopted for substrate conversion. It is noteworthy that these differences affect both the ATP binding pockets and the NAAD/NAD binding pockets and are therefore indicative of a structural communication between these sites during catalysis.

Comparison to the NAAD/NAD Binding Site of bsuNADSRegion 1, pointed out in the global comparison above (Fig. $4 A$ ), provides the docking environment for the adenylyl moiety in the NAAD/NAD binding site (Fig. $4 C$ ). Its amino acid composition is decisively different in ecoNADS and bsuNADS (Figs. 2 and $4 C$ ). E. coli region 1 contains a higher number of bulky, hydrophobic, or aromatic residues: Tyr-37 instead of Thr, Pro-38 instead of Gly, a Phe-39 insertion and Ile-40 instead of Ala (Figs. 2 and $4 C$ ). Despite these chemical differences, similar binding of the adenylyl moiety of NAAD or NAD is achieved by the two enzymes. Because of the phylogenetic variability in region 1 , the NAAD/NAD pocket may be of particular interest for the development of species-specific antibiotics. Additional minor variations at the NAD binding environment, such as the replacement of Val-156 and Trp-256 in ecoNADS by Leu-153 and Arg-253 in bsuNADS, could be of further relevance for rational inhibitor design.

We have outlined above how the N-terminal portion of loop P2 (residues 208-224) is involved in nucleotide binding at the ATP site through coordination of the $\mathrm{Mg}^{2+}-\mathrm{I}$ ion. In $b s u$ NADS, Ala-209 and Leu-211 from the same region of the P2 loop 
FIG. 5. Structural rearrangements. $A$, close-up stereo view of the ATP binding pocket of the ecoNADS apoenzyme (carbon atoms are in brown, and other atoms are colored as described in the legend to Fig. 4) superimposed onto the same region of the AMP complex structure (carbons in light blue). Residues lining the binding pocket are labeled in single letter style, and Arg-82 (R82) is highlighted. The arrow indicates repositioning of the Arg-82 side chain upon nucleotide binding. The dashed lines indicate hydrogen bonds of Arg-82 and of Gln-88 to the adenine base of AMP. $B, F-F_{c}$ difference omit map contoured at the $2.5 \sigma$ level with a radius of $3 \AA$ around NAAD (top) and NAD (bottom). The cofactors are shown as sticks. Arrows around NAAD bonds indicate the main rotations necessary to produce the NAD conformation. The arrow in NAD indicates the nitrogen of the amide group introduced in the course of the reaction. $C$, comparison of the NAAD/NAD binding pocket when complexed with NAD (carbon atoms and backbones traces in light blue) and when complexed with NAAD (carbon atoms and backbones traces in brown) after global superpositioning of the enzyme structures. Other atoms are color-coded as above. $\mathrm{Mg}^{2+}-\mathrm{I}$ is indicated as a green sphere coordinated to the Asp-51 and Glu-165 carboxylate groups and to water molecules (small red spheres). One water molecule also hydrogen bonds to the carboxylate of NAAD (dashed line). The different conformations of regions 224228, C-terminal of the disordered loop P2, are indicated by the backbone traces with side chains.
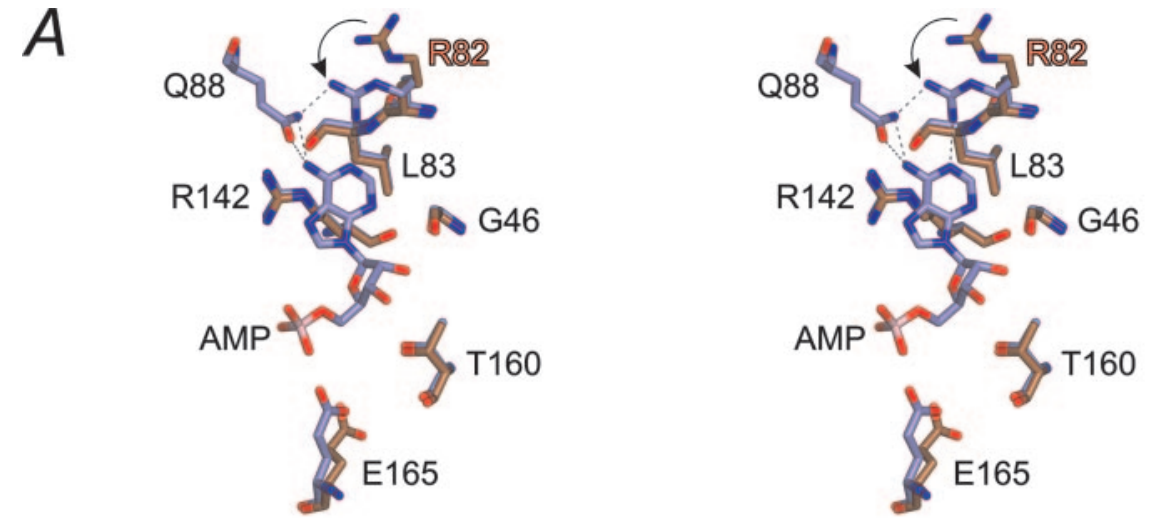

$B$

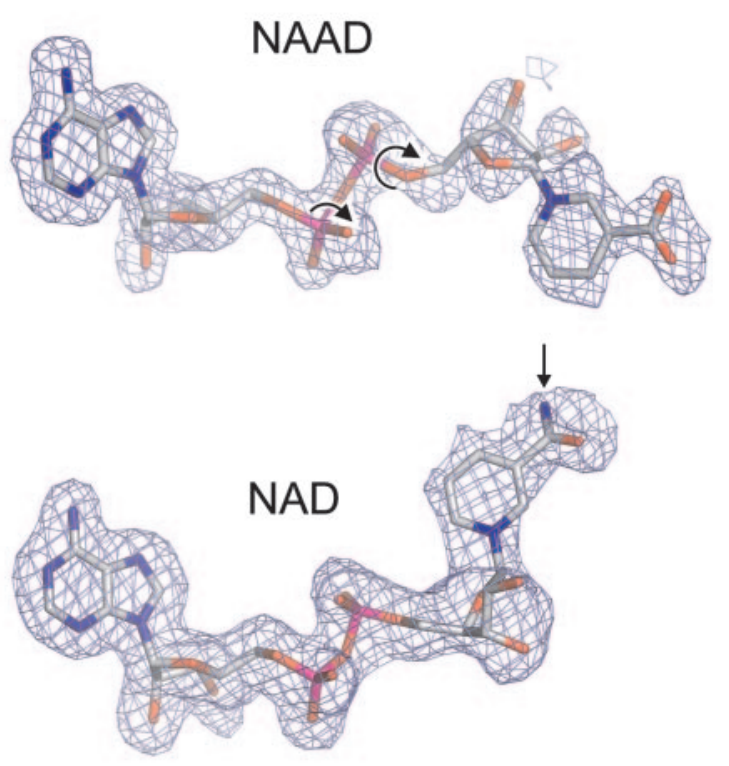

C
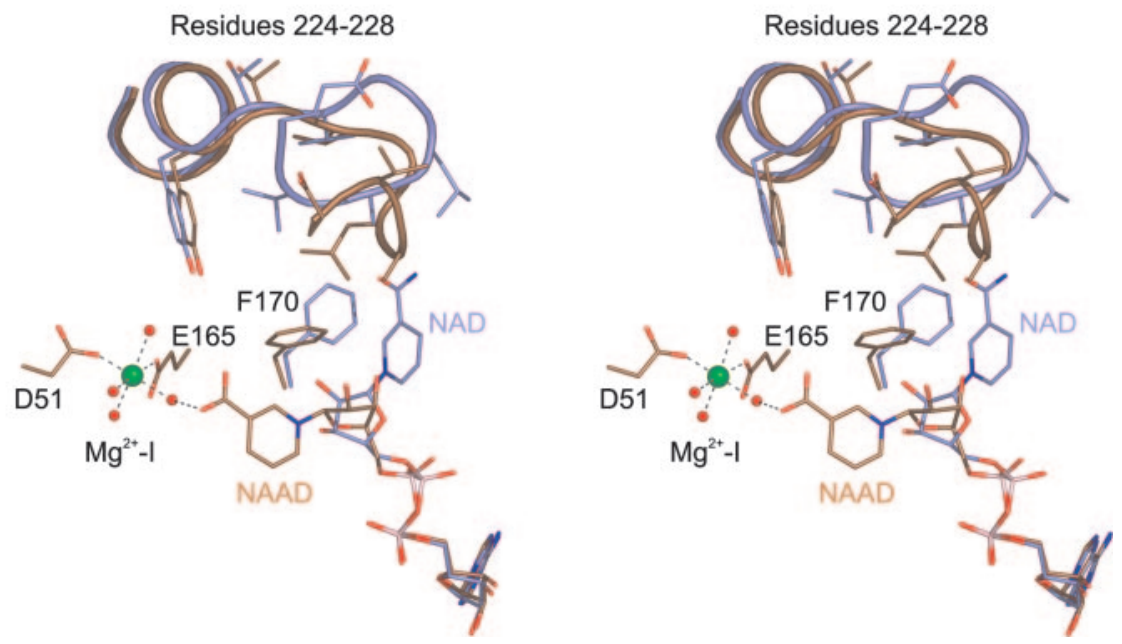

stabilize the nicotinysyl of bound NAAD. These interactions are not seen in ecoNADS. Supported by the crystal packing (see above), these interactions may therefore contribute to the fixation of loop P2 in bsuNADS structures. Interestingly, the C-terminal part of loop $\mathrm{P} 2$ also participates in substrate binding in bsuNADS. It is involved in the interaction with the nicotinysyl moiety of NAD-adenylate in the Bacillus enzyme (PDB code $1 \mathrm{KQP}$ ). The diol of the nicotinysyl ribose hydrogen bonds to the carboxyl group of Glu-223. Glu-223 is substituted by alanine in the $E$. coli enzyme, which consequently loses the interaction site to the nicotinysyl ribose.

\section{DISCUSSION}

In the present manuscript, we presented the high resolution crystal structures of ecoNADS as well as the structures of the enzyme with various substrates and products. The ensemble of 
structures encompasses the first co-crystal structure of a NADS with its enzymatic product, NAD. The structures confirm and significantly expand the picture that has emerged from the structural studies of $b s u$ NADS and provide evidence for a wide ranging conformational plasticity in this enzyme family. This plasticity manifests itself in (i) structural reorganizations upon binding of different substrates, (ii) a conformational cross-talk between the ATP binding site and the NAAD/ NAD binding pocket, and (iii) conformational adjustments during substrate conversion.

Flexible Elements Mediate Structural Reorganizations upon Substrate Binding-The mode of ATP binding appears to be largely invariant between $E$. coli and B. subtilis NADS. In both enzymes, loop P1 becomes fixed upon nucleotide binding to the ATP pocket and thus functions as an evolutionarily conserved lid for the ATP binding site. However, a significant difference between the two enzymes is seen in residue Arg-82, which undergoes side chain reorganization upon $\mathrm{ATP}$ or $\mathrm{AMP}+\mathrm{PP}_{\mathrm{i}}$ binding in ecoNADS, whereas its ATP-bound conformation is preformed in the bsuNADS apoenzyme. Thus, Arg-82 represents a hitherto unrecognized dynamical part of the ATP binding site in addition to loop P1.

Different from loop P1, the P2 loop is disordered in all structures of ecoNADS presented herein. In contrast, it is ordered in various bsuNADS structures, where several of its residues engage in direct or indirect contacts to the substrates ATP and NAAD. In its fixed conformation in $b s u$ NADS, both the N- and the C-terminal ends of the P2 loop are involved in substrate binding. At the $\mathrm{N}$ terminus, the backbone carbonyl of Thr- $208^{\text {bsu }}$ coordinates to $\mathrm{Mg}^{2+}$-II in the ATP pocket. In addition, residues Ala-209 ${ }^{b s u}$ and Leu-211 ${ }^{b s u}$ participate in the positioning of the nicotinysyl moiety of NAAD (15). At the $\mathrm{C}$ terminus, both side chain carboxyl oxygens of Glu-223 ${ }^{b s u}$ hydrogen bond to O-2' and $\mathrm{O}-3^{\prime}$ of the nicotinysyl ribose of nucleotides in the NAAD/NAD pocket. The highly similar sequence of the loop in ecoNADS and the similar modes of substrate binding suggest that P2 can interact in a similar fashion with the substrates in the $E$. coli protein, except for Glu-223 $3^{b s u}$ (which is replaced by alanine), but that these substrate interactions are transient. Thus, the main function of loop P2 seems to be the mediation of access to the substrate binding pockets and perhaps shielding them from the environment in the course of the reaction. Complete ordering of this loop in $b s u$ NADS structures seems to be observed, because it is supported by favorable crystal lattice contacts.

ecoNADS Dynamics in Response to Substrates and ProductsOur data provide the first co-crystal structure of a bacterial NADS in complex with the reaction product, NAD. We have shown that the nicotinysyl moiety of enzyme-bound NAD exhibits higher flexibility than the adenosyl portion and prefers a conformation, which is different from that of NAAD. The conformational difference in the substrate and product coincides with alternate conformations of Phe-170 and residues 224-228 following the C-terminal end of the disordered loop P2. Phe-170 stacks on the nicotinysyl ribose of NAAD and on the nicotinamide ring of NAD in the co-crystal structures. The conformation of residues 224-228 in the NAAD complex sterically interferes with the conformation of bound NAD (Fig. $5 C$ ). These observations suggest a structural reorganization prior to or accompanying product release. Catalysis requires an orientation of nicotinic acid toward the ATP binding site (14). We speculate that product release requires a torsion within $\mathrm{NAD}$, which in turn necessitates the reorganization of Phe-170 and the $\mathrm{P} 2$ loop. A possible trigger for this reorganization may be the $\mathrm{Mg}^{2+}-\mathrm{I}$ ion. In contrast to the previously reported complex structure of $b s u$ NADS with NAAD (15), $\mathrm{Mg}^{2+}-\mathrm{I}$ is present in our ecoNADS-NAAD co-crystal structure. The nicotinysyl carboxylate group of NAAD hydrogen bonds to a water of the $\mathrm{Mg}^{2+}$-I coordination sphere. On the other hand, $\mathrm{NAD}$ in the form $\mathrm{V}$ structure points away from the $\mathrm{Mg}^{2+}$-I site. Thus, $\mathrm{Mg}^{2+}-\mathrm{I}$ seems to act as an orientation device for the NAAD nicotinysyl moiety, which is activated by adenylyl transfer from ATP in the course of the reaction. Because $\mathrm{Mg}^{2+}-\mathrm{I}$ under physiological conditions presumably binds to the enzyme in complex with ATP, it prepares the NAAD conformation for this adenylyl transfer when the ATP pocket is ready to deliver the AMP moiety.

The data presented herein for ecoNADS, including the conservation of the mode of substrate and metal ion binding, are in general agreement with the reaction mechanism described for bsuNADS (16), suggesting that it is conserved for bacterial NADSs. However, our ensemble of ecoNADS structures allows us to expand the mechanism to structural changes taking place in the enzymes upon substrate conversion. We have found that the active center of ecoNADS exists in different conformations depending on the occupation of the ATP and NAAD/NAD binding sites. We refer to these conformations as the catalysis (or C-) state and the substrate/product exchange (or E-) state. The C-state is displayed in three of our crystal structures (forms II, III, IV), i.e. when ecoNADS is complexed with $\mathrm{AMP}, \mathrm{AMP}+\mathrm{PP}_{\mathrm{i}}$, or NAAD. The E-state predominates in the apoform of ecoNADS (form I) and in the complex with NAD (form V). The two states are distinguished by three major features: (i) the side chain conformation of Phe-170, (ii) the conformation of the P2 loop as represented by its C-terminal extension, comprising residues 224-228, and (iii) the occupation of the first magnesium site $\left(\mathrm{Mg}^{2+}-\mathrm{I}\right)$. The two states reflect the structural requirements in pursuing two interdependent processes. The E-state allows substrate binding and product release, and the $\mathrm{C}$-state provides the environment for adenylation and amidation of the substrate NAAD. As described above, $\mathrm{Mg}^{2+}$-I may be the element crucial for switching between the two states, because it seems to orient the NAAD nicotinysyl moiety. Following the enzymatic reaction, $\mathrm{AMP}, \mathrm{PP}_{\mathrm{i}}$, and $\mathrm{Mg}^{2+}-\mathrm{I}$ are released, shifting the equilibrium toward the E-state.

The NAAD/NAD Binding Pocket May Be a Target Site for Future Design of Species-specific Antibiotics-NADS has been proposed to be a promising target for the development of antibiotics to cure e.g. anthrax $(17,18)$. Knowledge of the results of structural comparisons among NADS from different organisms may allow development of inhibitory compounds that exhibit organism specificity. Although the structures of ecoNADS expectedly revealed a similar overall fold as bsuNADS (Figs. 3 and $4 A$ ), significant structural differences can be observed in detail. In particular, although the architectural alterations in the NAAD/NAD binding sites of E. coli and B. subtilis NADS do not affect the binding of the natural substrate, their chemically quite different makeup may provide a handle for specific inhibitor design (Fig. 4C).

In contrast to bacterial NADSs, which use exclusively ammonia as the amide donor (5, 7), NADS1 from human uses both ammonia and glutamine (11) (the ammonia-specific human NADS2 was later found to derive from a bacterial contamination (9)). Although many catalytic residues are invariant, human NADS1 contains several amino acid substitutions and peptide insertions at the ATP and the NAAD/NAD binding sites (Fig. 2). These differences confirm the suitability of the NADS enzymes for the design of antibiotics, because it should be possible to alleviate side effects in humans.

Acknowledgments-We thank Petra Birle and Tatjana Krywcun for help in enzyme preparation and crystallization, Dr. Hans-Georg Beisel and Dr. Peter Hof for initial help in structure solution, and Prof. Dr. Herbert Jäckle for support in data analysis. 


\section{REFERENCES}

1. Rizzi, M., and Schindelin, H. (2002) Curr. Opin. Struct. Biol. 12, 709-720

2. Preiss, J., and Handler, P. (1958) J. Biol. Chem. 233, 488-492

3. Preiss, J., and Handler, P. (1958) J. Biol. Chem. 233, 493-500

4. Spencer, R. L., and Preiss, J. (1967) J. Biol. Chem. 242, 385-392

5. Nessi, C., Albertini, A. M., Speranza, M. L., and Galizzi, A. (1995) J. Biol. Chem. 270, 6181-6185

6. Willison, J. C. (1992) J. Bacteriol. 174, 5765-5766

7. Willison, J. C., and Tissot, G. (1994) J. Bacteriol. 176, 3400-3402

8. Cantoni, R., Branzoni, M., Labo, M., Rizzi, M., and Riccardi, G. (1998) J. Bacteriol. 180, 3218-3221

9. Bieganowski, P., and Brenner, C. (2003) J. Biol. Chem. 278, 33056-33059

10. Bieganowski, P., Pace, H. C., and Brenner, C. (2003) J. Biol. Chem. 278, 33049-33055

11. Hara, N., Yamada, K., Terashima, M., Osago, H., Shimoyama, M., and Tsuchiya, M. (2003) J. Biol. Chem. 278, 10914-10921

12. Rizzi, M., Nessi, C., Mattevi, A., Coda, A., Bolognesi, M., and Galizzi, A. (1996) EMBO J. 15, 5125-5134

13. Rizzi, M., Nessi, C., Bolognesi, M., Coda, A., and Galizzi, A. (1996) Proteins 26, $236-238$

14. Rizzi, M., Bolognesi, M., and Coda, A. (1998) Structure (Camb.) 6, 1129-1140

15. Devedjiev, Y., Symersky, J., Singh, R., Jedrzejas, M., Brouillette, C., Brouil- lette, W., Muccio, D., Chattopadhyay, D., and DeLucas, L. (2001) Acta Crystallogr. Sect. D 57, 806-812

16. Symersky, J., Devedjiev, Y., Moore, K., Brouillette, C., and DeLucas, L. (2002) Acta Crystallogr. Sect. D 58, 1138-1146

17. Sutherland, S. (2003) Drug Discov. Today 8, 335-336

18. Velu, S. E., Cristofoli, W. A., Garcia, G. J., Brouillette, C. G., Pierson, M. C., Luan, C. H., DeLucas, L. J., and Brouillette, W. J. (2003) J. Med. Chem. 46, 3371-3381

19. Schoepfer, R. (1993) Gene 124, 83-85

20. Otwinowski, Z., and Minor, W. (1996) Methods Enzymol. 276, 307-326

21. Navaza, J. (2001) Acta Crystallogr. Sect. D 57, 1367-1372

22. Brunger, A. T., Adams, P. D., Clore, G. M., DeLano, W. L., Gros, P., GrosseKunstleve, R. W., Jiang, J. S., Kuszewski, J., Nilges, M., Pannu, N. S., Read, R. J., Rice, L. M., Simonson, T., and Warren, G. L. (1998) Acta Crystallogr. Sect. D 54, 905-921

23. McRee, D. E. (1999) J. Struct. Biol. 125, 156-165

24. Laskowski, R. A., MacArthur, M. W., Moss, D. S., and Thornton, J. M. (1993) J. Appl. Crystallogr. 26, 283-291

25. Collaborative Computational Project, N. (1994) Acta Crystallogr. Sect. D 50, $760-763$

26. Jones, S., and Thornton, J. M. (1996) Proc. Natl. Acad. Sci. U. S. A. 93, 13-20

27. Wallace, A. C., Laskowski, R. A., and Thornton, J. M. (1995) Protein Eng. 8 , 127-134 


\section{Supplementary Chapter IV: The zinc finger-associated domain of the Drosophila transcription factor Grauzone is a novel zinc-coordinating protein-protein interaction module}

\section{Citation:}

Jauch, R., Bourenkov, G. P., Chung, H. R., Urlaub, H., Reidt, U., Jackle, H., and Wahl, M. C. (2003). The zinc finger-associated domain of the Drosophila transcription factor grauzone is a novel zinc-coordinating protein-protein interaction module. Structure (Camb) 11, 1393-1402.

\section{Statement of Contribution:}

cloning, protein expression, protein purification, biochemical assays, light scattering, circular dichroism, structure refinement, model, building, structure analysis, manuscript preparation 


\section{The Zinc Finger-Associated Domain of the Drosophila Transcription Factor Grauzone Is a Novel Zinc- Coordinating Protein-Protein Interaction Module}

\author{
Ralf Jauch, ${ }^{1}$ Gleb P. Bourenkov, ${ }^{3}$ Ho-Ryun Chung, ${ }^{1}$ \\ Henning Urlaub, ${ }^{2}$ Ulrich Reidt, ${ }^{2}$ Herbert Jäckle, ${ }^{1, *}$ \\ and Markus C. Wahl' \\ ${ }^{1}$ Max-Planck Institut für biophysikalische Chemie \\ Abteilung Molekulare Entwicklungsbiologie \\ ${ }^{2}$ Abteilung Zelluläre Biochemie/ \\ Röntgenkristallographie \\ Am Faßberg 11 \\ D-37077 Göttingen \\ ${ }^{3}$ MPG-ASMB c/o DESY \\ Arbeitsgruppe Proteindynamik \\ Notkestraße 85 \\ D-22603 Hamburg \\ Germany
}

\section{Summary}

About one-third of the more than $300 \mathrm{C} 2 \mathrm{H} 2$ zinc finger proteins of Drosophila contain a conserved sequence motif, the zinc finger-associated domain (ZAD). Genes that encode ZAD proteins are specific for and expanded in the genomes of insects. Only three ZADencoding gene functions are established, and the role of ZAD is unknown. Here we present the crystal structure of the ZAD of Grauzone $\left(Z A D_{\text {Grau }}\right)$, a Drosophila transcription factor that specifically controls the maternal Cdc20-like APC subunit Cortex. ZAD forms an atypical treble-clef-like zinc-coordinating fold. Headto-tail arrangement of two $Z A D_{\text {Grau }}$ molecules in the crystals suggests dimer formation, an observation supported by crosslinking and dynamic light scattering. The results indicate that ZAD provides a novel protein-protein interaction module that characterizes a large family of insect transcription factors.

Introduction

A large number of protein domains, collectively referred to as zinc fingers, bind zinc ions through various combinations of four cysteine and/or histidine residues (Berg and Shi, 1996; Klug and Schwabe, 1995). The coordination of a zinc ion allows such modules to adopt compact three-dimensional structures which are otherwise too small to maintain extensive hydrophobic cores (Grishin, 2001). The prototype zinc finger proteins contain a 30 amino acid C2H2 motif (Miller et al., 1985; Pavletich and Pabo, 1991). In addition to this motif, a number of variants were found which are distinguished by zinccoordinating residues, the secondary structural elements contributing the zinc ligands and/or topology (Krishna et al., 2003; Laity et al., 2001). While a large number of zinc fingers function as DNA binding domains (Pavletich and Pabo, 1991; Rosenberg et al., 1986; Wolfe et al., 2000), it becomes increasingly clear that they can also mediate interactions of the protein with RNA, other

*Correspondence: hjaeckl@gwdg.de proteins and small molecules of other kinds (Krishna et al., 2003; Mackay and Crossley, 1998; McCarty et al., 2003). Furthermore, zinc-coordinating folds may confer specific enzymatic activities on proteins (Lorick et al., 1999).

The $\mathrm{C} 2 \mathrm{H} 2$ zinc finger motif characterizes one of the most abundant eukaryotic protein families (Lander et al., 2001). $\mathrm{C} 2 \mathrm{H} 2$ zinc finger proteins frequently contain additional protein modules in their $\mathrm{N}$ termini (Collins et al., 2001) such as the mammalian Krüppel-associated box (KRAB) or the insect zinc finger associated domain (ZAD) (Lespinet et al., 2002). Both KRAB- and ZAD-containing $\mathrm{C} 2 \mathrm{H} 2$ zinc finger proteins are characterized by lineage-specific expansions in the respective genomes (Chung et al., 2002; Lespinet et al., 2002; Looman et al., 2002). The KRAB domain acts as a transcriptional repressor module (Margolin et al., 1994; Peng et al., 2000), whereas the function of ZAD is unknown.

In silico studies have recently identified more than 90 ZAD proteins in Drosophila, accounting for $\sim 28 \%$ of the total $\mathrm{C} 2 \mathrm{H} 2$ zinc finger proteins of this organism (Chung et al., 2002). This family of proteins is characterized by a conserved constellation of four cysteines within the ZAD, the chromosomal clustering of the corresponding genes, and the lack of homologs in noninsect genomes (Chung et al., 2002). Although the identification of expressed sequence tags ensured that the majority of ZAD-encoding genes of the Drosophila genome are transcribed, only four annotated members of the gene family have been examined in some detail. Two of them, Serendipity delta (Sry- $\delta)$ and grauzone (grau), were shown to encode transcriptional activators of the eggpolarity gene bicoid (Payre et al., 1994) and the gene cortex which encodes a Cdc20-like APC subunit, respectively (Chen et al., 2000). The third factor, the Dorsal-interacting protein 1 (DIP1), was identified in a yeast two-hybrid screen as an interaction partner of the dorsoventral polarity transcription factor Dorsal (Bhaskar et al., 2000). Finally, Zeste-white-5 (Zw5) was reported to confer enhancer-blocking activity by association with the boundary element scs and may thereby participate in chromatin structuring by providing an insulating activity (Blanton et al., 2003; Gaszner et al., 1999). grau, Zw5, and $S r y-\delta$ have also been characterized by mutant analysis showing that they carry vital functions (Chen et al., 2000; Crozatier et al., 1992; Gaszner et al., 1999). In two cases, single amino acid replacements within the ZAD, such as cysteine 7 by tyrosine in Sry- $\delta$ (Crozatier et al., 1992) and arginine 4 by glycine in Zw5 (Gaszner et al., 1999), were the cause of lethality, implying that conserved amino acids within the ZAD are essential for its function.

Here we present the structure and biochemical features of the ZAD of Grauzone (ZAD $\left.D_{\text {Grau }}\right)$. Grauzone is a 570 amino acid long transcriptional regulator characterized by the $\mathrm{N}$-terminal ZAD and an array of eight C-terminal $\mathrm{C} 2 \mathrm{H} 2$ zinc finger domains which mediate the binding of Grauzone to a promotor element of its target gene cortex (Chen et al., 2000). In genetic terms, grau 
is necessary for the proper transcriptional activation of the gene cortex, coding for a Cdc20-like APC subunit (Chu et al., 2001). Maternal loss-of-function mutations of grau cause an irregular growth arrest in meiosis II, whereas homozygous grau mutant embryos, which have received maternal grau activity, develop into normal adults. The grau mutant phenotype suggests that grau is exclusively required during oogenesis and cortex is its only target gene (Chen et al., 2000). Our results show that $Z A D_{\text {Grau }}$ exhibits a $C 4$ zinc-coordinating fold with a novel treble-clef-like structure. The crystallographic data and additional biochemical evidence indicate that $Z A D_{\text {Grau }}$ is a protein interaction module with the capability to form homodimers and suggest that ZAD provides a taxon-specific means for the assembly of $\mathrm{C} 2 \mathrm{H} 2$ transcription factor complexes.

\section{Results and Discussion}

Sequence alignments of a total of 91 Drosophila $\mathrm{C} 2 \mathrm{H} 2$ proteins and corresponding proteins of a variety of other insects such as Anopheles gambiae revealed a conserved $\mathrm{N}$-terminal sequence motif that contains two invariant pairs of cysteines (Figure 1A) (for details see Chung et al., 2002). Due to the specific association with $\mathrm{C} 2 \mathrm{H} 2$ family members of zinc finger proteins, this motif was termed zinc finger-associated domain (ZAD). In order to characterize this protein motif, we studied the $Z A D$ of the transcription factor Grauzone $\left(Z A D_{\text {Grau }}\right)$. The $Z A D_{\text {Grau }}$ open reading frame (amino acid residues 2-90; Figure $1 \mathrm{~A}$ ) was fused to Glutathione S-transferase (GST) and the recombinant fusion protein was produced in bacteria (see Experimental Procedures). After an initial capture step, the $Z A D_{\text {Grau }}$ portion of the fusion protein was liberated by protease digestion, purified by column chromatography, and crystallized (see Experimental Procedures). SDS-PAGE analysis of dissolved crystals revealed that they contain only the $Z A D_{\text {Grau }}$ protein (data not shown). The fact that $Z A D_{\text {Grau }}$ crystallizes provides first evidence that ZADs encompass an independently folding protein module.

\section{ZAD Is a Zinc Binding Protein Module}

Based on the four conserved cysteine residues, ZADs have been suggested to comprise metal binding modules (Chung et al., 2002; Lander et al., 2001; Lespinet et al., 2002). Because metal association is expected to contribute significantly to the stability of the fold, heat denaturation experiments were performed in the presence and absence of EDTA. In the absence of EDTA, $Z A D_{\text {Grau }}$ was stable for at least $15 \mathrm{~min}$ at $60^{\circ} \mathrm{C}$, whereas in the presence of EDTA $(5 \mathrm{mM})$ a considerable portion of the protein precipitated when heated above $50^{\circ} \mathrm{C}$ for $15 \mathrm{~min}$ (Figure 1B). In these experiments, care was taken to exclude divalent metal ions from the purification procedure. The result therefore suggests that $Z A D_{\text {Grau }}$ contains an endogenous metal center that confers stability on its structure. In order to test this proposal, we performed an X-ray fluorescence scan. With this approach, the characteristic emission lines for zinc were observed (Figure 1C). This result and the finding that the anomalous diffraction from the zinc center could be exploited to solve the structure with a native crystal (see below) demonstrate that $Z A D_{\text {Grau }}$ includes a zinc ion.

\section{Structure Solution and Quality of the Model}

The primary sequence of $Z A D_{\text {Grau }}$ is shown in Figure $1 A$. The crystal structure of $Z A D_{\text {Grau }}$ was solved de novo by a two-wavelength multiple anomalous dispersion experiment around the K-edge of an endogenous $\mathrm{Zn}^{2+}$ ion. $\mathrm{A}$ segmented poly-alanine model could be manually fitted to the experimental electron density map and allowed the subsequent incorporation of side chains. The sequence assignment was guided by well-defined aromatic residues and by the two pairs conserved cysteines (C4, C7, C53, and C56; see Figures $1 A$ and 2A), which coordinated the $\mathrm{Zn}^{2+}$ ion (Figure 1D). Three regions in flexible loops (V11-C17, D22-E27, and E41-E47; see Figures $1 \mathrm{~A}$ and $2 \mathrm{~A}$ ) initially displayed weak densities and were fitted during the subsequent rounds of refinement and manual model building. These loop regions, which are made up of nonconserved residues, lack substantial intra- and interchain contacts. Thus, they gained aboveaverage temperature factors during the refinement. The main chain electron density of the molecule remained fragmented around position S12-A14 and D22 in the final $2 F_{0}-F_{c}$ map, consistent with high internal flexibility and lack of crystal packing contacts in these regions. All other side chains of the refined model, except those of some hydrophilic surface residues, were entirely covered by the final $2 \mathrm{~F}_{\mathrm{o}}-\mathrm{F}_{\mathrm{c}}$ map. No electron density developed for the C-terminal 9 residues (positions 82-90; Figure $1 \mathrm{~A}$ ) of $Z A D_{\text {Grau }}$ and the $5 \mathrm{~N}$-terminal vector-derived residues that are contained in the recombinant protein. The final model therefore encompasses the residues D2-S81 of ZAD ${ }_{\text {Grau }}$ that could be traced unambiguously. Presumably because of the considerable fraction of flexible residues at the termini, which could not be accounted for in the final model, the refinement converged with an $\mathrm{R}$ factor/ $\mathrm{R}_{\text {free }}$ factor of $24.1 \% / 26.7 \%$. Convergence at these numbers is consistent with a rather high B factor extracted from a Wilson plot $\left(52.6 \AA^{2}\right)$, which is comparable to the averaged $B$ factor of the final structure $\left(52.9 \AA^{2}\right)$.

During all refinement steps, $5 \%$ of the reflections were set aside to monitor the $\mathrm{R}_{\text {free }}$ factor (Table 1). Of the final model, $91.9 \%$ of the residues resided in the preferred regions of the Ramachandran plot, $6.8 \%$ in the additionally allowed areas. Only the single residue K29, which was well defined in both the experimental and the final model-derived maps assumed an unconventional $\phi / \psi$ conformation even after manual interference. The mean positional error of the model was estimated at $0.15 \AA$ (Luzzati, 1952).

Structural Properties of $Z A D_{\text {Grau }}$

The structured portion of $Z A D_{\text {Grau }}$ resembles the letter "b." Its approximate dimensions are $60 \times 30 \times 30 \AA$ (Figures $2 \mathrm{~A}$ and 2B). The N-terminal body of the "b" comprises a globular fold structured around a zinc ion. The C-terminal stem is formed by a long $\alpha$ helix ( $\alpha 2$, positions 54-80) that contains almost one-third of all residues of the domain. Residues 82-90 are not included in the ZAD consensus sequence (Chung et al., 2002) 
A

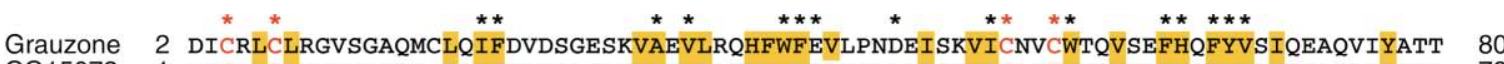

CG15073 1 MICRLCLNNVNDTDT-IRIFEDVGLSLNVANVLAKYFWFEPKSDDPISTVICLTCWNQVNDFHQFYVAVESAHRLLTER 79

A. gambiae 1 RKCTACFS-FSESEY-LNIFAEENVDKDIATVIAMHLWFEVTPTDEC-QWICRTCWTSLDSFHSFYVSIEQRHERHTDK 78 $\Delta \mathrm{A}$
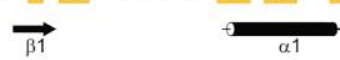

$\Delta A$

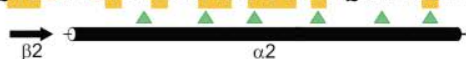

B

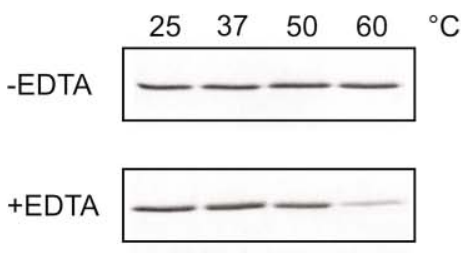

D

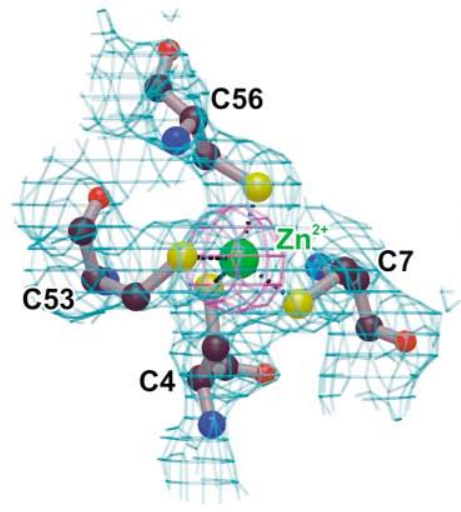

C

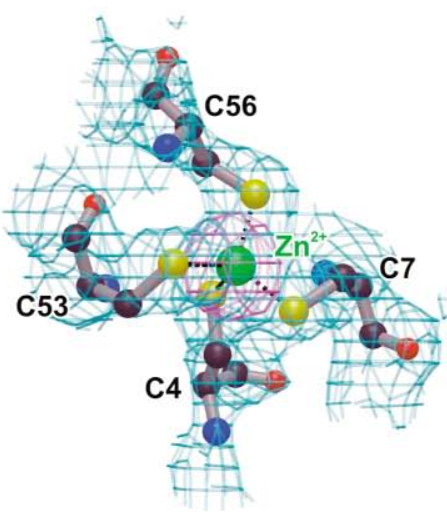

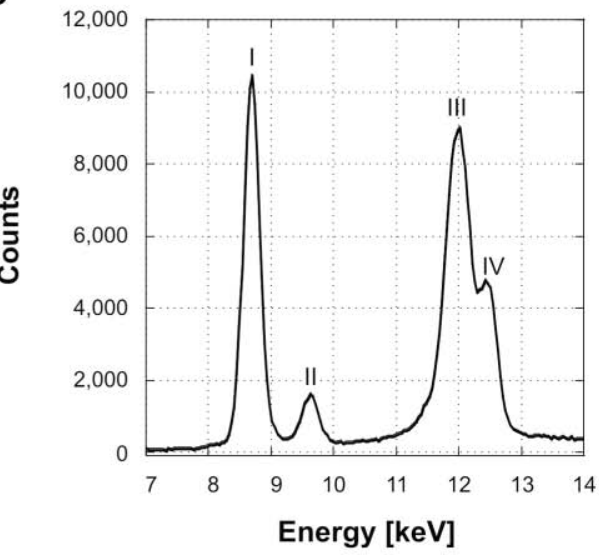

Figure 1. ZAD as a Zinc-Coordinating Fold

(A) Alignment of the Grauzone ZAD with its closest paralog, CG15073, in the Drosophila melanogaster genome and with a putative Anopheles gambiae homolog (ZAD only fragment, see Experimental Procedures). Asterisks mark 100\% conservation in all three ZADs; red characters, invariant cysteine pairs; yellow boxes, conserved hydrophobic or aromatic amino acid residues.

(B) Portion of $Z A D_{\text {Grau }}$ remaining soluble after incubation at the indicated temperatures in the absence (-EDTA) and in the presence of 5 mM EDTA (+EDTA). Reduction of the soluble fraction is clearly visible when $Z A D_{\text {Grau }}$ is heated above $50^{\circ} \mathrm{C}$ in presence of the chelator.

(C) X-ray emission spectrum of a native $Z A D_{\text {Grau }}$ crystal irradiated with X-radiation of $\lambda=1.0 \AA$. I, ZnK $\alpha$ line; II, ZnK $\beta$ line; III, Compton scattering; IV, elastic scattering.

(D) Electron density maps around the zinc center in $Z^{2} D_{\text {Grau. }}$ Cyan, experimental MAD electron density map ( $\left.1 \sigma\right)$; magenta, anomalous difference Fourier map $(10 \sigma)$, generated with the anomalous differences at the peak wavelength and the phases obtained after solvent flattening. The zinc-coordinating cysteine residues are labeled and shown in ball-and-stick. If not mentioned otherwise, all structural figures were prepared with Bobscript (http://www.strubi.ox.ac.uk/bobscript/) and rendered with Raster3D (Merritt and Bacon, 1997).

and are disordered in the present structure. Presumably, they provide a flexible linker to the remainder of the molecule, i.e., an acidic region followed by an array of eight $\mathrm{C} 2 \mathrm{H} 2$ zinc fingers in the case of Grauzone (Chen et al., 2000).

The arrangement of the different ZAD structural elements is summarized in Figures $2 A-2 C$ and detailed in the Figure 2 legend. The fold of $Z A D_{\text {Grau }}$ appears to be critically dependent on the zinc coordination, a result that is consistent with the EDTA-dependent precipitation of $Z A D_{\text {Grau }}$ shown above. The two pairs of coordinating cysteines are approximately 50 residues apart. Zinc coordination, therefore, links the $\beta 2-\alpha 2$ transition region at the center of the molecule with the $\mathrm{N}$ terminus of the domain likely to solidify the structure. In this view, the position of the zinc ion allows the fold to constrain the slanting angle of the C-terminal helix, $\alpha 2$, with respect to the long axis of the $\beta$ sheet (Figures 2B-2C). The relative orientation of this long helix and the $\beta$ sheet is further defined by helix $\alpha 1$, which rests with one of its surfaces on the end of the $\beta$ sheet distal to the zinc ion and the adjacent loop regions. With a neighboring surface, helix $\alpha 1$ is in contact with the $\mathrm{N}$-terminal and central portions of helix $\alpha 2$. Both the $\alpha 1$ sheet and the $\alpha 1-\alpha 2$ associations are based on extensive hydrophobic contacts forming a considerable hydrophobic core $(\alpha 1$ : V29, V33, L34, H37, and F38; $\beta 1 / \beta 2$ : L18, I20, and I52; $\alpha 2$ : W57, V60, F63, H64, and Y67; Figures $1 A$ and 2A-2C). In addition, the conserved $\mathrm{H} 37$ residue (atom NE2) of helix $\alpha 1$ hydrogen bonds at the edge of this core to the 
A

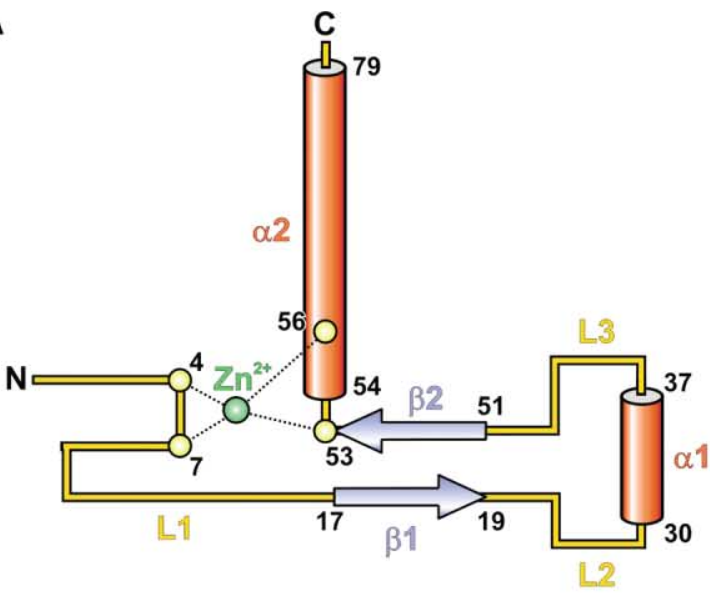

B

C

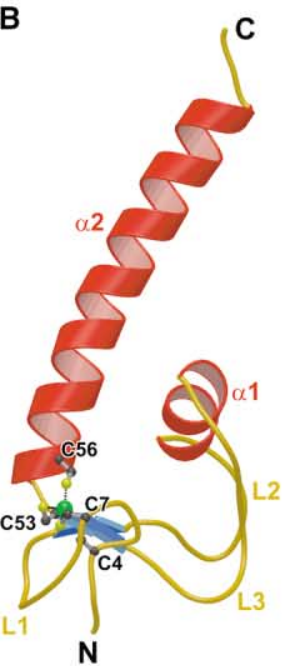

$\downarrow$

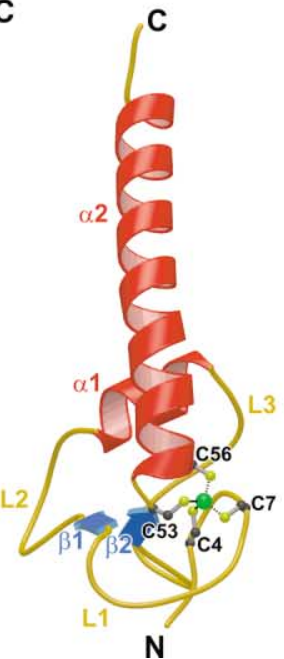

N
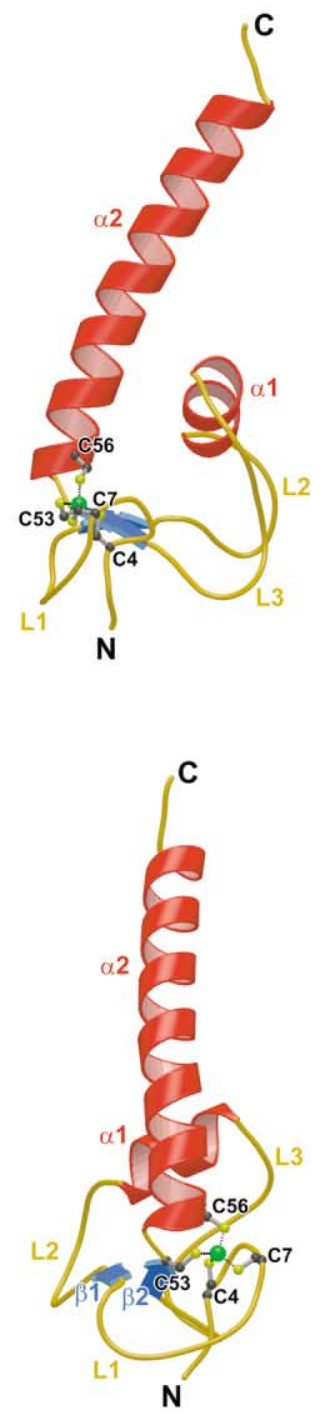

Figure 2. ZAD Structure and Topology

( $A$ and $B$ ) Two orthogonal stereo ribbon plots of the $Z A D_{\text {Grau }}$ structure. $\alpha$ helices, red; $\beta$ strands, blue; loops, gold. In the side view of (A), the structure resembles the letter "b." $\mathrm{N}$ - and $\mathrm{C}$ termini, secondary structure elements, and residues of the zinc center are labeled. The $\mathrm{Zn}^{2+}$ ion (green) and its coordinating cysteine side chains are shown in ball-and-stick. conserved H64 (atom ND1) of helix $\alpha 2$. In conclusion, the $L 2 / \alpha 1 / L 3$ region between the two central $\beta$ strands and the zinc ion provide platforms which orient the C-terminal helix relative to the globular portion of the $\mathrm{ZAD}_{\text {Grau }}$ molecule.

To further support the overall structure of $Z A D_{\text {Grau }}$ obtained by X-ray crystallography (Figures 2A-2D), we took circular dichroism spectra of the molecule in solution (data not shown). The calculated secondary structure content (45\%-50\% helix, 5\%-10\% strands) obtained from these recordings compare favorably to those seen in the crystal structure ( $37 \%$ helix, $6 \%$ strands). The conservation of length, the predicted secondary structure, the arrangement of the annotated secondary structure elements and the critical amino acid residues of ZADs (Chung et al., 2002) (Figure 1A) suggest that the present $Z A D_{\text {Grau }}$ crystal structure provides a prototype for ZAD folding.

\section{Structure Comparison}

Next we compared the ZAD fold with known structures by manual inspection, with special emphasis on reported zinc finger fold groups (Krishna et al., 2003). We found that ZAD exhibits a structural relation to treble clef zinc finger (TCZF) domains. Figure $3 A$ shows a comparison of ZAD with the structure of the C-terminal C4 TCZF of chicken GATA-1 (PDB ID code: 2gat) (Tjandra et al., 1997), revealing a similar topology of the secondary structure elements. The treble clef element has been identified among the members of seven different SCOP (http://scop.berkeley.edu/) fold groups including RING fingers, LIM domains, ribosomal proteins L24E and S14, and nuclear receptor-like fingers (Grishin, 2001; Krishna et al., 2003). Thus, despite the lack of significant sequence similarity between ZADs and TCZFs, which prevented recognition of their relation in the past, ZAD can be regarded as a novel, atypical TCZF that exerts several distinct features. Unlike the canonical zinc knuckle of

(C) Topological diagram of the $Z A D_{\text {Grau }}$ structure identifying the origin of the four zinc-coordinating cysteines and the borders of the secondary structure elements (numbers).

(D) Alignment of $10 \mathrm{ZAD}$ sequences. Sequence numbering corresponds to that of $Z A D_{\text {Grau. }}$. Highly conserved residues (Chung et al., 2002) are shown on a golden background. Green triangles above the alignment indicate residues involved in dimer formation (see also Figure 4). Secondary structure elements as seen in the $Z A D_{\text {Grau }}$ structure are indicated below the alignment. Note that the $\mathrm{N}$ terminus of $Z A D_{\text {Grau }}$ is folded into a hairpin motif (D2-V11) and contains two conserved zinc-coordinating cysteine residues (C4 and C7; Figure1A). Replacement of $\mathrm{C} 7$ by a tyrosine residue has been described as a mutation of Sry- $\delta$ (Crozatier et al., 1992), implying that $C 7$ is essential for ZAD function directly involved in zinc coordination. The $\mathrm{N}$-terminal hairpin is followed by a loop (L1: S12-M16) which extends into a two-stranded antiparallel $\beta$ sheet ( $\beta 1$ : C17-Q19; $\beta 2$ : V51-C53) that is interspersed by a long loop-helix-loop insertion (L2: I20-K29; $\alpha 1$ : V30-H37; L3: F38-K50) and followed by a C-terminal helix, $\alpha 2$. This helix is directly linked to the second $\beta$ strand with a slanting angle of $\sim 80^{\circ}$ between the helix axis and the average long axis of the $\beta$ sheet. Helix $\alpha 1$ of the L2- $\alpha 1$-L3 motif is positioned perpendicular to both the long axis of the sheet and the axis of helix $\alpha 2$, wedging between the two motifs. Conserved $\mathrm{C} 53$ and $\mathrm{C} 56$ residues reside in the C-terminal part of strand $\beta 2$ and the first turn of helix $\alpha 2$, respectively, and complete the zinc coordination sphere. 


\begin{tabular}{|c|c|c|}
\hline Dataset & Remote & Peak \\
\hline \multicolumn{3}{|l|}{ Data Collection } \\
\hline Spacegroup & $\mathrm{P} 4,2,2$ & \\
\hline \multicolumn{3}{|l|}{ Unit cell lengths $(\AA)$} \\
\hline a & 48.7 & \\
\hline b & 48.7 & \\
\hline c & 82.1 & \\
\hline Wavelength $(\AA)$ & 1.0500 & 1.2828 \\
\hline Resolution (Å) & $50.0-2.0$ & $50.0-2.5$ \\
\hline Unique Reflections & 6907 & 6089 \\
\hline Redundancy & 4.6 & 2.6 \\
\hline Completeness (\%) & $97.0(99.7)$ & $96.3(98.5)$ \\
\hline l/_(l) & $36.7(6.3)$ & $28.2(14.2)$ \\
\hline $\mathbf{R}_{\text {sym }}{ }^{a}(\%)$ & $4.2(44.7)$ & $3.1(8.2)$ \\
\hline \multicolumn{3}{|l|}{ Phasing } \\
\hline Resolution (Å) & $20.0-2.1$ & \\
\hline Heavy atom sites & 1 & \\
\hline \multicolumn{3}{|l|}{$\mathbf{R}_{\text {Cullis }}{ }^{b}$} \\
\hline Centrics & 0.50 & \\
\hline Acentrics & 0.61 & \\
\hline \multicolumn{3}{|l|}{ Phasing power } \\
\hline Centrics & 1.67 & \\
\hline Acentrics & 1.97 & \\
\hline \multicolumn{3}{|l|}{$\mathrm{FOM}^{d}$} \\
\hline Before DM & 0.42 & \\
\hline After DM & 0.78 & \\
\hline \multicolumn{3}{|l|}{ Refinement } \\
\hline Resolution (Å) & $15.0-2.0$ & \\
\hline Number of reflections & 6590 & \\
\hline \multicolumn{3}{|c|}{ Number of nonhydrogen atoms } \\
\hline Protein & 644 & \\
\hline $\mathrm{Zn}^{2+}$ ions & 1 & \\
\hline Water oxygens & 56 & \\
\hline $\mathbf{R}_{\text {work }}{ }^{e}(\%)$ & $24.1(29.6)$ & \\
\hline $\mathbf{R}_{\text {free }}{ }^{e}(\%)$ & $26.7(33.7)$ & \\
\hline \multicolumn{3}{|c|}{ Rmsd from ideal geometry } \\
\hline Bond lengths (Å) & 0.009 & \\
\hline Bond angles $\left({ }^{\circ}\right)$ & 1.20 & \\
\hline \multicolumn{3}{|l|}{ Average B factors $\left(\AA^{2}\right)$} \\
\hline Protein & 50.6 & \\
\hline $\mathrm{Zn}^{2+}$ ion & 37.2 & \\
\hline Water oxygens & 80.3 & \\
\hline Wilson B factor & 52.6 & \\
\hline \multicolumn{3}{|l|}{ Rmsd B factors $\left(\AA^{2}\right)$} \\
\hline Main chain bonds & 1.9 & \\
\hline Main chain angles & 3.3 & \\
\hline Side chain bonds & 3.5 & \\
\hline Side chain angles & 5.2 & \\
\hline \multicolumn{3}{|c|}{ Ramachandran analysis (\%) } \\
\hline Preferred & 91.9 & \\
\hline Additionally allowed & 6.8 & \\
\hline Disallowed & 1.4 & \\
\hline Mean residual error (Å) & 0.15 & \\
\hline
\end{tabular}

Data for the last $0.05 \AA$ in parentheses. DM, density modification (solvent flattening); rmsd, root-mean-square deviation.

${ }^{\mathrm{a}} \mathbf{R}_{\text {sym }}(\mathrm{l})=\left(\Sigma_{\mathrm{hk}} \Sigma_{i}\left[\left|l_{i}(\mathrm{hkl})-<l(\mathrm{hkl})>\right|\right] / \Sigma_{\mathrm{hk}} \Sigma_{i}\left[l_{i}(\mathrm{hkl})\right] ; l_{i}(\mathrm{hkl})-\right.$ intensity of the $i^{\text {th }}$ measurement of hkl; $</($ hkl) $>-$ average value of hkl for all $i$ measurements.

${ }^{\mathrm{b}} \mathrm{R}_{\text {Cullis }}=\Sigma_{\mathrm{hk}}\left[|| F_{\mathrm{PH}} \pm F_{\mathrm{P}}|-| F_{\mathrm{H}, \text { calc }} \mid\right] / \Sigma_{\mathrm{hk}}\left[\left|F_{\mathrm{PH}} \pm F_{\mathrm{P}}\right|\right] ;\left(F_{\mathrm{PH}}+F_{\mathrm{P}}\right)$ if signs are opposite, $\left(F_{\mathrm{PH}}-F_{\mathrm{P}}\right)$ if equal.

${ }^{c}$ Phasing power $=\left(\Sigma_{\mathrm{n}}\left[\left|F_{\mathrm{h}}\right|^{2}\right] / \Sigma_{\mathrm{n}}\left[|E|^{2}\right]\right)^{1 / 2} ; \Sigma_{\mathrm{n}}\left|E^{2}\right|=$ lack of closure error $=$ $\Sigma_{\mathrm{n}}\left[\left|F_{\mathrm{PH}}\right|(\text { obs })-\left|F_{\mathrm{PH}}\right|(\mathrm{calc})\right]^{2}$

${ }^{\mathrm{d}} \mathrm{FOM}=$ figure of merit $\left.\left.=[\mid F(\mathrm{hk}))_{\text {best }} \mid\right] / F(\mathrm{hk})\right) ; ; \mathrm{F}(\mathrm{hk})_{\text {best }}=\Sigma_{\alpha}\left[P(\alpha) F_{\text {hkl }}(\alpha)\right]$ $/ \Sigma_{\alpha}[P(\alpha)]$.

$\left.{ }^{e} R_{\text {work }}=\Sigma_{\text {hkl }}\left[|| F_{\text {obs }}|-k| F_{\text {calc }}||\right] / \Sigma_{\text {hkl }}\left[\left|F_{\text {obs }}\right|\right] ; R_{\text {free }}=\Sigma_{\text {hkl } 1 T}\left[|| F_{\text {obs }}|-k| F_{\text {calc }}||\right]\right]$

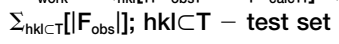
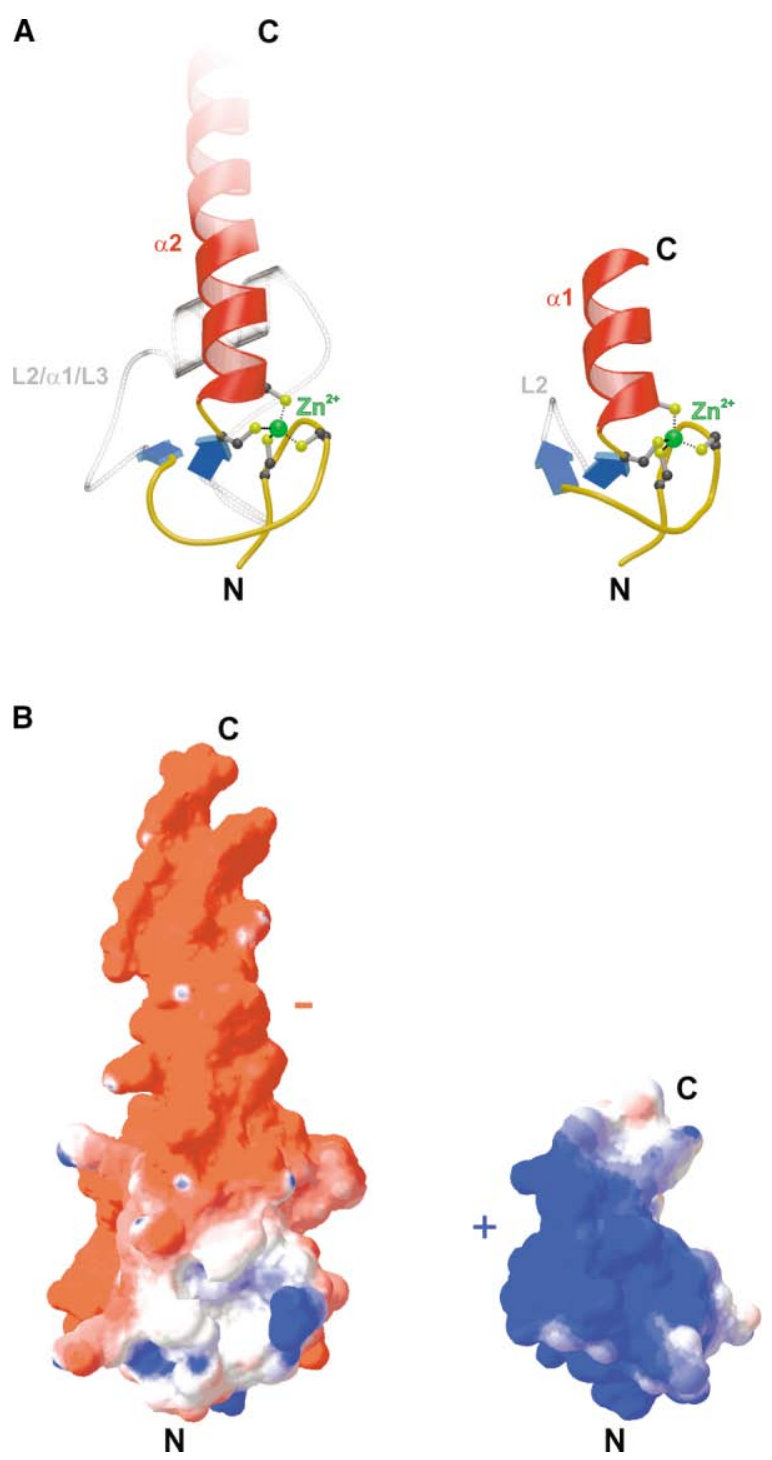

Figure 3. Structural Comparison

Ribbon (A) and electrostatic surface representation $(B)$ of $Z A D_{\text {Grau }}$ (left panels) and the C-terminal TCZF from chicken GATA-1 (right panels). Color coding for the ribbon plot is as in Figure 2. In the surface plot, red indicates negative potential, blue positive potential. The orientation is the same as in Figure 2B. This and all other surface images were prepared with SwissPDBViewer (Guex and Peitsch, 1997).

the TCZFs (Grishin, 2001), the ZAD N-terminal hairpin lacks the CPXCG consensus and an extended $\beta$ conformation at both ends. Instead, the residue following the first cysteine in ZAD is a conserved arginine, and the loop is stabilized by only one backbone-to-backbone hydrogen bond. Furthermore, in a typical TCZF the two central $\beta$ strands are connected by a terse loop, whereas in $Z A D$, the corresponding loop is expanded into the L2/ $\alpha 1 /$ L3 motif. In addition, helix $\alpha 2$ of ZAD comprises six turns and thus is considerably longer than the corresponding helices of TCZFs, which typically contain a maximum of four turns only (Grishin, 2001). As detailed above, insertion of helix $\alpha 1$ via the L2/ $\alpha 1 /$ L3 motif may 


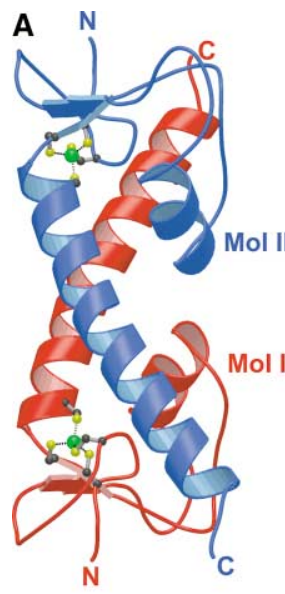

C

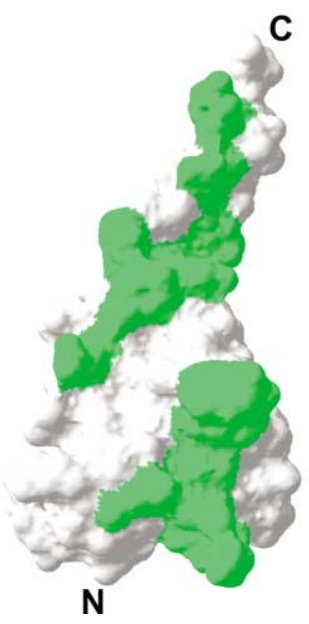

C
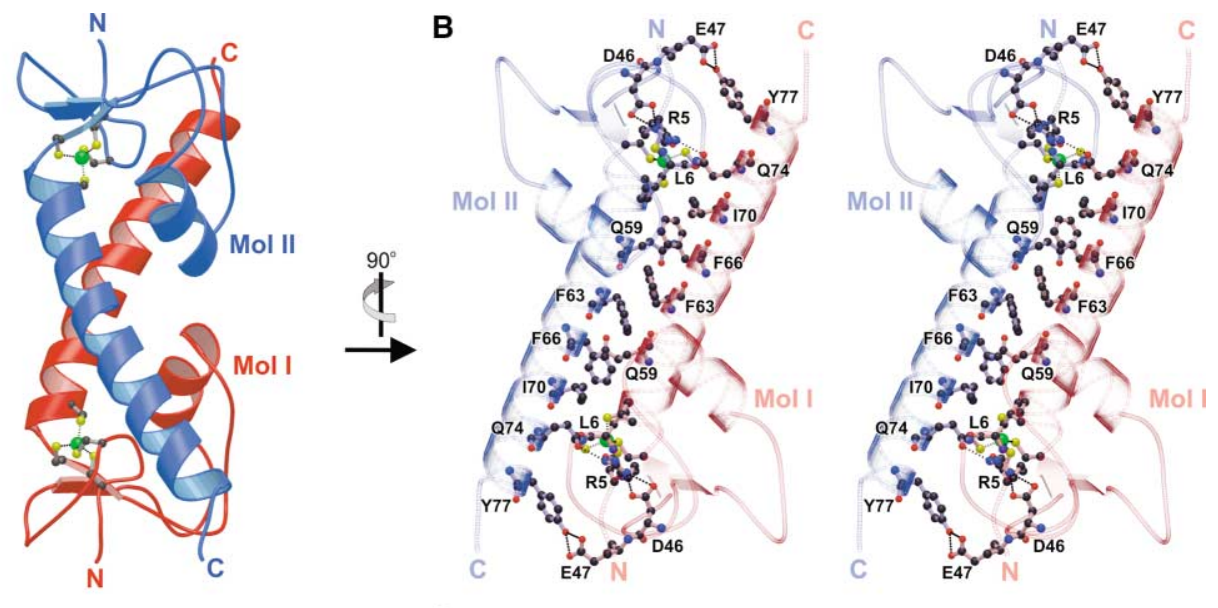

D
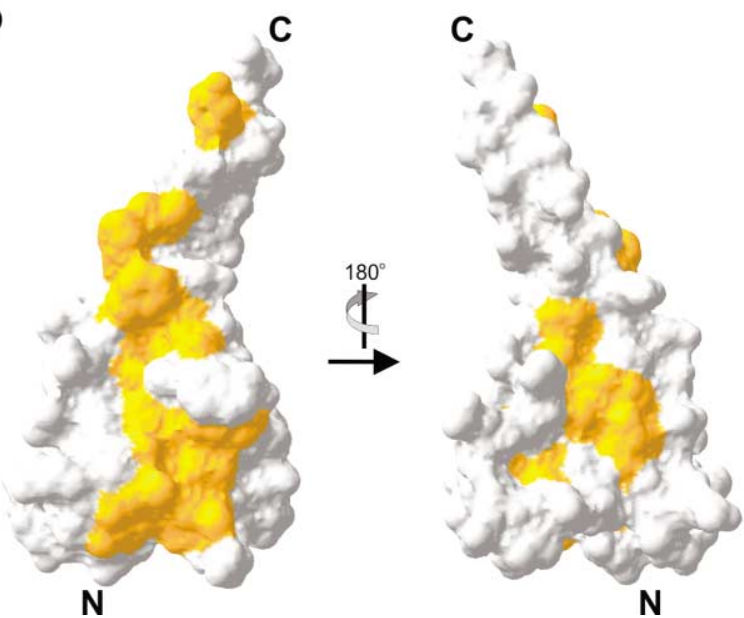

Figure 4. The $Z A D_{\text {Grau }}$ Dimer

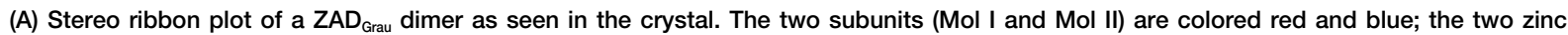
centers are depicted in ball-and-stick. $\mathrm{N}$ - and $\mathrm{C}$ termini are labeled. The orientation of the rear subunit is the same as in Figure $2 \mathrm{~A}$.

(B) Stereo representation of residue interactions in the dimer interface, $90^{\circ}$ from the view in (A). The two $Z A D_{\text {Grau }}$ subunits are colored as in (A) but are rendered semitransparent to clearly reveal the interacting residues in ball-and-stick. All residues and the molecular termini are labeled.

(C) Mapping of the contact residues within the dimer (green) onto the surface of a $Z_{A D}$ Grau monomer (gray). The orientation of the molecule is the same as that of the red subunit in (A).

(D) Mapping in orange of conserved residues on the surface of $Z A D_{\text {Grau }}$ in two diametric views. The left orientation is the same as in (C), indicating that the largest conserved surface patch and the dimer interface largely coincide.

be a prerequisite for extending the length of helix $\alpha 2$, without loosing its defined orientation relative to the $\beta$ sheet. Finally, helix $\alpha 2$ displays a conserved pattern of hydrophobic residues in ZAD (Figure 1D; see also below) which is absent from other TCZFs. ZAD can therefore be classified as a distinct subgroup of TCZF domain structures.

Crystal Structure Suggests $Z A D_{\text {Grau }}$ Homodimers

The evolutionary restriction of ZADs to certain $\mathrm{C} 2 \mathrm{H} 2$ zinc finger genes in insects, the chromosomal clustering of the majority of these ZAD-containing genes and the unique folding characteristics of the domain next to the DNA binding domain of transcriptional regulators such as Grauzone strongly argue that the ZAD is associated with a specific and distinct biological function. However, the mere similarity to the fold group of the TCZFs does not allow any conclusions about the specific role of ZAD, because treble clef motifs embody functions as diverse as binding to nucleic acids, proteins as well as small ligands and some may even exert enzymatic activity (Grishin, 2001).

However, the crystal structure of $Z A D_{\text {Grau }}$ clearly supports a model, in which ZAD represents a protein-protein interaction module involved in homodimerization. Figure 4 shows that in the crystal two $Z A D_{\text {Grau }}$ molecules are associated through a 2-fold axis in an isologous headto-tail fashion. As revealed by the protein-protein interaction server (http://www.biochem.ucl.ac.uk/bsm/PP/ server/), this contact buries $\sim 1000 \AA^{2}$ of accessible surface area $(\triangle \mathrm{ASA})$. This value seems on the lower side when compared to known homodimers (Jones and Thornton, 1996). However, the interaction surface of $Z A D_{\text {Grau }}$ covers a total of close to $20 \%$ of the entire surface area. More importantly, a large number of amino acid residues, which are strongly conserved among ZAD 
A

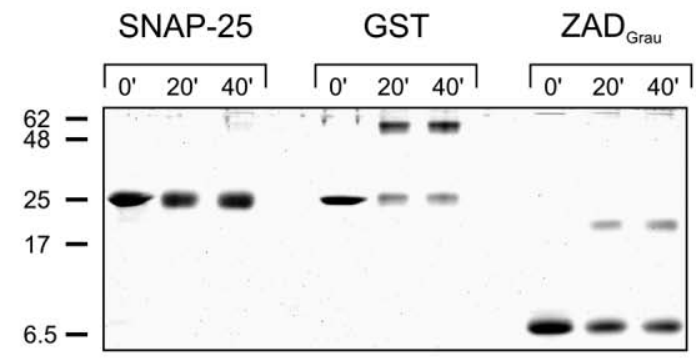

B

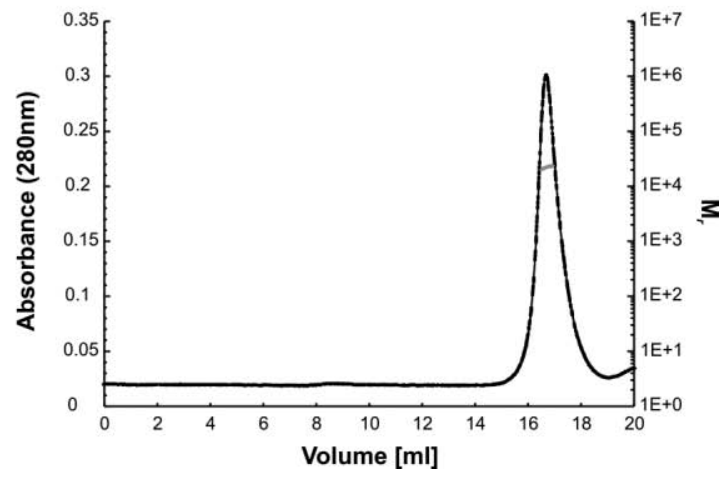

Figure 5. Detection of $Z A D_{\text {Grau }}$ Dimers in Solution

(A) Results from chemical crosslinking with glutaraldehyde displayed on a denaturing polyacrylamide gel. Crosslinking took place for the times indicated above the lanes. SNAP-25 served as a negative control, GST as a positive control. Molecular weight markers are given on the left $(\mathrm{kDa})$.

(B) Results from a gel filtration chromatography run combined with multiangle-laser-light scattering analysis of the emerging peak. Emergence of protein from the column was detected by absorbance at $280 \mathrm{~nm}$ (black trace, left $y$ axis), readout from the light scattering of the single, symmetrical peak is depicted as a gray line (right $y$ axis).

family members (for details see Chung et al., 2002), are responsible for the contacts between the two subunits (Figure 4B). When the conserved residues are mapped on the surface of $Z A D_{\text {Grau, }}$ it becomes obvious that the largest conserved surface patch closely coincides with the presumed dimer interface (Figures 4C and 4D). In particular, hydrophobic residues of the long C-terminal helix $\alpha 2$ (F63, F66, 170, and Y77; Figure 1A) build up major parts of the contact interface (Figure 4B). As a consequence, $72.5 \%$ of the amino acid residues of the dimer interface are nonpolar. The presumed dimer interface is thus designed very differently from the remainder of the surface, which is lined with polar residues and exhibits a highly negative electrostatic potential (Figure 3B). In the region where the tip of helix $\alpha 2$ from one molecule contacts the globular portion of the other subunit, some intermolecular hydrogen bonds are observed as well (Q74-R5; Y77-E47; Figure 4B).

Because of the involvement of its hydrophobic side chains, the presumed homodimerization mode provides a straightforward explanation for the amphipathic design and the unusual length of helix $\alpha 2$ (Figure 2A). Since, in addition, both helix $\alpha 2$ and the globular portion of ZAD are involved in this dimerization mode, the importance of restraining their relative orientations by the inserted L2/ $\alpha 2 / L 3$ module and the zinc coordination becomes obvious. Further supporting its significance, the present dimerization may explain the lethal phenotype observed with the conserved R4 (corresponding to R5 in Grauzone) mutated to glycine in Zw5 (Gaszner et al., 1999). Its side chain is positioned by ionic interactions with D46 to engage in a hydrogen bond with Q74 of the neighboring molecule (Figure 4). We note, however, that there are a number of other contacts between the two subunits that could stabilize subunit interactions. It is therefore possible that the mutation simply perturbs the globular structure of the $\mathrm{N}$ terminus which might preclude dimerization. In any case, the analysis of the crystal packing strongly suggests a functional homodimerization of $Z A D_{\text {Grau. }}$
Besides the above symmetrical contact between two $Z A D_{\text {Grau }}$ molecules, other crystal contacts were observed. Some of these are not functional because of an unreasonably small $\triangle$ ASA. Other contacts did not involve conserved residues. One rather intimate alternative association takes place through the crystallographic 4-fold screw axis. However, this symmetry element gives rise to heterologous contacts, leaving the bonding potentials of ZAD unsaturated. The latter interaction mode could therefore lead to the formation of larger oligomers. We therefore examined the arrangement of ZAD in solution.

\section{$Z_{\text {Grau }}$ Forms Dimers in Solution}

The quaternary structure of $Z A D_{\text {Grau }}$ in solution was investigated by two approaches. First, we performed chemical crosslinking experiments with glutaraldehyde (Figure 5A). In contrast to SNAP-25, which was reported not to self-interact (Fasshauer et al., 1999), and in parallel with the dimerizing GST, ZAD ${ }_{\text {Grau }}$ could be efficiently crosslinked to the dimer state, but no higher oligomers were observed. Secondly, multiangle-laser-light-scattering following size exclusion chromatography yielded strong evidence for homdimerization in solution (Figure $5 B) . Z A D_{\text {Grau }}$ eluted as a single symmetrical peak from various size exclusion columns with different optimal separation regimes. Comparison of the elution times with those of reference proteins was consistent with $Z A D_{\text {Grau }}$ dimers (data not shown). The scattering signal at $632.8 \mathrm{~nm}$ across the elution peak indicated a molecular weight of $21.11 \mathrm{kDa}$, which matched the theoretical dimer mass (21.14 kDa) almost perfectly (Figure 5B). This high congruence and the lack of a monomer or a trimer signal, strongly suggested a monodisperse dimer solution.

Collectively, our results provide strong evidence for $Z A D_{\text {Grau }}$ homodimerization under near-physiological salt conditions. They leave the 2 -fold symmetrical association of $Z A D_{\text {Grau }}$ in the crystal (Figure 4) as the only dimerization mode, which is consistent with all observations. 
Because $Z A D_{\text {Grau }}$ is an independently folding unit, dimerization is expected to prevail within the context of the full-length Grauzone transcription factor. By extension of this finding, a general function of ZADs could be to provide dimerization modules that mediate homodimer and/or heterodimer formation among closely related members of the ZAD transcription factor family. In support of this observation, homodimerization was also reported for the ZAD-containing transcription factor Sry- $\delta$ (Payre et al., 1997; Ruez et al.,1998). It is noteworthy that Sry- $\delta$ comprises a deviated version of ZAD family members and could thus not be considered as prototype for ZAD function (Chung et al., 2002).

\section{ZAD $_{\text {Grau }}$ Lacks DNA Binding Features}

The majority of zinc finger proteins were shown to be nucleic acid binding proteins (Berg and Shi, 1996; Laity et al., 2001; Pavletich and Pabo, 1991; Wolfe et al., 2000). Because of this amply documented function, we asked whether the $Z A D_{\text {Grau }}$ structure has features to support nucleic acid binding as well. In order to obtain first hints, we compared the ZAD with the structure of the C-terminal treble clef module of the chicken erythroid transcription factor GATA-1 (Tjandra et al., 1997), a representative of sequence-specific DNA binding TCZFs. Figure 3B shows that the two molecules display opposite electrostatic surface potentials. Whereas the TCZF of GATA-1 exhibits an almost continuously electropositive surface potential that facilitates a close association with the negatively charged sugar-phosphate backbone of the $D N A$, the $Z A D_{\text {Grau }}$ is almost completely wrapped into negative potential. This observation strongly argues against DNA binding features of ZAD.

Superimposition of one subunit of a $Z A D_{\text {Grau }}$ dimer on the structure of DNA-bound GATA-1 TCZF (Tjandra et al., 1997) revealed that the second $Z A D_{\text {Grau }}$ module faces the minor groove of the DNA and would thus collide with the DNA backbone (data not shown). Furthermore, Grauzone mutants, which lack the ZAD-containing region of the protein, had unaltered DNA binding properties in vitro, whereas the part of Grauzone protein that contains the ZAD in the absence of $\mathrm{C} 2 \mathrm{H} 2$ zinc fingers had no DNA binding activity (Chen et al., 2000), supporting the argument that $Z A D_{\text {Grau }}$ has no DNA binding features. Since the key positions and features of the amino acid residues are conserved among ZADs (for an alignment, see Chung et al., 2002), it appears unlikely that any of them carries DNA binding properties. We therefore propose that ZADs, in general, are protein interaction modules involved in homodimerization and/or the formation of protein complexes. To further support this conclusion, we also compared the $Z A D_{\text {Grau }}$ dimer with the DNA-bound dimer of other members of the TCZF fold group, the glucocorticoid receptors (Luisi et al., 1991; Grishin, 2001). In these receptor molecules, the DNA binding helix equivalent to ZAD helix $\alpha 2$ is employed as a recognition element, which is positioned in the DNA major groove. Two DNA binding domains interact through their C-terminal helical extension, which have no equivalent in ZADs. These $\mathrm{C}$-terminal extensions yield a spacing between the two recognition helices from neighboring subunits, which is large enough to position them both into neighboring turns of the DNA major groove. Therefore, the dimerization mode of ZADs is fundamentally different from the interaction mode seen in the DNA binding domains of glucocorticoid receptors, consistent with these molecules serving different functions.

\section{Experimental Procedures}

\section{Sample Preparation and EDTA-Dependent Precipitation}

The DNA coding for Grauzone ZAD residues 2-90 was PCR amplified, cloned into the Not1/EcoR1 sites of the pGEXT-3 vector, and recombinantly expressed as a GST-fusion protein in a BL21(DE3) $E$. coli strain. Cells were grown to an $\mathrm{OD}_{600} 0.7-1.0$ at $30^{\circ} \mathrm{C}$, shifted to $20^{\circ} \mathrm{C}$, and induced with $0.7 \mathrm{mM}$ IPTG overnight. Cells were harvested by centrifugation and resuspended in phosphate-buffered saline containing Complete protease inhibitor tabs (Roche) and traces of lysozyme. Cells were lysed by sonication and the insoluble fraction was removed by centrifugation. GST-ZAD $\mathrm{Grau}_{\text {was }}$ waptured using Glutathione (GSH)-Sepharose 4B (Amersham) beads in disposable columns following the manufacturer's suggestions. Samples were eluted with $50 \mathrm{mM}$ Tris- $\mathrm{HCl}, \mathrm{pH}$ 8.0, $50 \mathrm{mM}$ GSH. Thrombin was directly added to the eluate to $1 \mathrm{U} / 100 \mu \mathrm{g}$ protein and incubated overnight at room temperature. The protein identity was confirmed by peptide finger printing and subsequently purified with a $1 \mathrm{ml}$ Ressource-Q $15 \mu \mathrm{m}$ anion exchange column applying a linear 20 $\mathrm{ml}$ salt gradient $(20 \mathrm{mM}$ Tris- $\mathrm{HCl}, \mathrm{pH} 8.0,0-1 \mathrm{M} \mathrm{NaCl})$, a second GSH column and a Superdex 25/60 75 pg gel filtration (GF) column (GF buffer: $10 \mathrm{mM}$ Tris, pH 7.5, $100 \mathrm{mM} \mathrm{NaCl}, 5 \mathrm{mM}$ DTT). Samples were concentrated to $8 \mathrm{mg} / \mathrm{ml}$ via $20 \mathrm{ml} 5000 \mathrm{Da}$ molecular weight cutoff concentrators (Vivascience). For EDTA-dependent precipitation studies, $10 \mu \mathrm{M} Z \mathrm{ZAD}_{\text {Grau }}$ samples in GF buffer either lacking EDTA or containing $5 \mathrm{mM}$ EDTA were incubated for $15 \mathrm{~min}$ at room temperature, $37^{\circ} \mathrm{C}, 50^{\circ} \mathrm{C}$, and $60^{\circ} \mathrm{C}$. Samples were pelleted and the supernatant was analyzed by SDS-PAGE.

\section{Crystallization and Data Collection}

Crystallization screens were carried in the sitting drop vapor diffusion format on 24-well Cryschem plates (Hampton Research, Laguna Niguel). 1-4 $\mu \mathrm{l}$ of an $8 \mathrm{mg} / \mathrm{ml} Z_{\text {GA }}$ solution in GF buffer were combined with $1 \mu \mathrm{l}$ reservoir buffer and equilibrated against a $400 \mu \mathrm{l}$ reservoir. Crystals grew at $22^{\circ} \mathrm{C}$ with $0.2 \mathrm{M}$ ammonium acetate, $0.1 \mathrm{M}$ sodium citrate, $\mathrm{pH} 5.6$, and $30 \%$ polyethylene glycol 4000 in 3-10 days.

After transfer into Paratone-N (Hampton Research) and removal of residual mother liquor, crystals could be shock frozen in a liquid nitrogen stream. Diffraction data were collected at $100 \mathrm{~K}$ on the HASYLAB beamline BW6 (DESY, Hamburg, Germany; http://www-hasylab.desy. de/facility/experimental_stations/stations/BW6.htm). X-ray fluorescence spectra of native crystals clearly indicated zinc emission lines (Figure 1B). A single such crystal yielded complete anomalous data sets at the $\mathrm{f}^{\prime \prime}$-maximum of the K-edge $(\lambda=1.2828 \AA)$ and at a remote wavelength $(\lambda=1.0500 \AA)$. The data were recorded on a MarResearch (Norderstedt, Germany) CCD detector and processed with the HKL package (Otwinowski and Minor, 1997) (see also Table 1).

Structure Solution, Model Building, and Refinement If not mentioned otherwise, programs from the CCP4 collection (CCP4, 1994) were employed for the structure solution. The location of a single $\mathrm{Zn}^{2+}$ ion could be deduced from anomalous difference Patterson maps at the $f^{\prime \prime}$-maximum (program RSPS) or by direct methods (SHELX, http://shelx.uni-ac.gwdg.de/SHELX/) and was used to phase the data with the remote data set as the reference (MLPHARE). The hand of the heavy atom was revealed during solvent flattening (DM).

Prominent secondary structure elements were manually fitted to the electron density with MAIN (Turk, 1996). Subsequently, these regions were decorated with side chains guided by the prominent features of the aromatic residues and by four cysteine residues, which coordinated the $\mathrm{Zn}^{2+}$ ion. The connecting loops were sequentially traced by alternating refinement of the partial model (CNS) (Brunger et al., 1998) and model building guided by the experimental 
and $2 \mathrm{~F}_{\mathrm{o}}-\mathrm{F}_{\mathrm{c}}$ electron density maps. Refinement included standard procedures of positional and $B$ factor optimization, a round of simulated annealing, and positioning of 56 water molecules into spherical peaks of the $F_{\mathrm{o}}-\mathrm{F}_{\mathrm{c}}$ maps. The final step was a TLS refinement with REFMAC5 with the structure divided into four rigid bodies (the $\mathrm{Zn}^{2+}$ ion, residues 2-19 and 51-54, residues 20-50, and residues 55-81). No data within 15.0-2.0 ̊ were excluded, and during all refinement steps the same set of reflections $(5 \%)$ was used to monitor the $R_{\text {tree }}$ factor (Table 1). The geometry of the final model was analyzed with PROCHECK (Laskowski et al., 1993) (Table 1), secondary structure elements were extracted with PROMOTIF (http://www.biochem. ucl.ac.uk/bsm/promotif/promotif.html).

\section{Spectroscopy}

For far UV-CD measurements, the sample was dialyzed against 10 $\mathrm{mM} \mathrm{Na} \mathrm{HPO}_{4}, \mathrm{pH}$ 7.9. The spectra $(190-260 \mathrm{~nm})$ were recorded with a JASCO J-720 spectropolarimeter (Jasco Corporation, Tokyo, Japan) at $20^{\circ} \mathrm{C}$ in a $0.1 \mathrm{~cm}$ cuvette with a scanning speed of $50 \mathrm{~nm} /$ $\mathrm{min} .30$ scans were averaged and baseline corrected. The spectra were analyzed using the neural network-based CD deconvolution software CDNN v2.1 (Böhm et al., 1992).

\section{Chemical Crosslinking}

The crosslinking reactions were carried out at $25^{\circ} \mathrm{C}$ in $20 \mathrm{mM}$ Tris$\mathrm{HCl}, \mathrm{pH} 7.5,100 \mathrm{mM} \mathrm{NaCl}$ with $15 \mu \mathrm{M}$ samples of $Z A D_{\text {Grau, }}$ GST (26 $\mathrm{kDa}$ ), and SNAP-25 (25 kDa) (Fasshauer et al., 1999) and $1 \mathrm{mM}$ glutaraldehyde. The reactions were stopped at selected time points by boiling in SDS sample buffer.

\section{Multiangle-Laser-Light Scattering}

The experiment was performed on a HR-10/30 Superdex-200 size exclusion column (Amersham) connected to a UV spectrometer and the Dawn and Optilab instruments XY (Wyatt Technology Corp.). $200 \mu \mathrm{l}$ of a $25 \mu \mathrm{M} Z^{2} D_{\text {Grau }}$ sample were chromatographed in 20 $\mathrm{mM}$ Tris- $\mathrm{HCl}, \mathrm{pH} 8.0,150 \mathrm{mM} \mathrm{NaCl}$. The $\mathrm{UV}_{280}$ absorption, the light scattering at $632.8 \mathrm{~nm}$ and the differential refraction of the elution profile were monitored. Spectra were analyzed with the Astra software package (Wyatt, 1993). We also checked $Z A D_{\text {Grau }}$ migration on an analytical Superdex-75 column in comparison to protein molecular weight standards.

\section{Identification of a Putative Grauzone ZAD Homolog} in the Anopheles gambiae Genome

We searched the whole Anopheles gambiae genome using the ZAD HMM described in Chung et al., 2002 and the Wise package 2.2.0 (Birney et al., 1996). All identified ZADs were aligned with all Drosophila melanogaster ZADs using ClustalW 1.8.1. (Thompson et al., 1994), false positive hits were eliminated. The alignment was used to construct a neighbor-joining tree with ClustalW. Based on this tree, we identified the closest relative of the Grauzone ZAD in the Anopheles gambiae genome. The alignment of the Grauzone ZAD with the closest paralog, CG15073, of Drosophila melanogaster and the putative Anopheles homolog was performed with ClustalW using default parameters.

\section{Acknowledgments}

We thank our colleagues in the labs for their various contributions, T. Siddiqui for kindly providing SNAP-25, G. Dowe for sequencing, and Stefan Pabst for help with MALLS analysis. Work was supported by the Max Planck Society. H.-R.C. is a fellow of the Boehringer Ingelheim Fonds.

Received: June 30, 2003

Revised: July 7, 2003

Accepted: July 9, 2003

Published: November 4, 2003

References

Berg, J., and Shi, Y. (1996). The galvanization of biology: a growing appreciation for the roles of zinc. Science 271, 1081-1085.

Bhaskar, V., Valentine, S., and Courey, A. (2000). A functional interac- tion between dorsal and components of the smt 3 conjugation machinery. J. Biol. Chem. 275, 4033-4040.

Birney, E., Thompson, J., and Gibson, T.J. (1996). PairWise and SearchWise: finding the optimal alignment in a simultaneous comparison of a protein profile against all DNA translation frames. Nucleic Acids Res. 24, 2730-2739.

Blanton, J., Gaszner, M., and Schedl, P. (2003). Protein:protein interactions and the pairing of boundary elements in vivo. Genes Dev. 17, 664-675.

Böhm, G., Muhr, R., and Jaenicke, R. (1992). Quantitative analysis of protein far UV circular dichroism spectra by neural networks. Protein Eng. 5, 191-195.

Brunger, A., Adams, P., Clore, G., DeLano, W., Gros, P., GrosseKunstleve, R., Jiang, J., Kuszewski, J., Nilges, M., Pannu, N., et al. (1998). Crystallography \& NMR system: a new software suite for macromolecular structure determination. Acta Crystallogr. D 54, 905-921.

Chen, B., Harms, E., Chu, T., Henrion, G., and Strickland, S. (2000). Completion of meiosis in Drosophila oocytes requires transcriptional control by grauzone, a new zinc finger protein. Development 127 , 1243-1251.

Chu, T., Henrion, G., Haegeli, V., and Strickland, S. (2001). Cortex, a Drosophila gene required to complete oocyte meiosis, is a member of the Cdc20/fizzy protein family. Genesis 29, 141-152.

Chung, H., Schäfer, U., Jäckle, H., and Böhm, S. (2002). Genomic expansion and clustering of ZAD-containing $\mathrm{C} 2 \mathrm{H} 2$ zinc-finger genes in Drosophila. EMBO Rep. 3, 1158-1162.

Collins, T., Stone, J., and Williams, A. (2001). All in the family: the BTB/POZ, KRAB, and SCAN domains. Mol. Cell. Biol. 21, 3609-3615. Crozatier, M., Kongsuwan, K., Ferrer, P., Merriam, J., Lengyel, J., and Vincent, A. (1992). Single amino acid exchanges in separate domains of the Drosophila serendipity delta zinc finger protein cause embryonic and sex biased lethality. Genetics 131, 905-916.

Fasshauer, D., Antonin, W., Margittai, M., Pabst, S., and Jahn, R. (1999). Mixed and non-cognate SNARE complexes. Characterization of assembly and biophysical properties. J. Biol. Chem. 274, 14401446.

Gaszner, M., Vazquez, J., and Schedl, P. (1999). The Zw5 protein, a component of the scs chromatin domain boundary, is able to block enhancer-promoter interaction. Genes Dev. 13, 2098-2107.

Grishin, N. (2001). Treble clef finger-a functionally diverse zincbinding structural motif. Nucleic Acid Res. 29, 1703-1714.

Guex, N., and Peitsch, M. (1997). SWISS-MODEL and the SwissPdbViewer: an environment for comparative protein modeling. Electrophoresis 18, 2714-2723.

Harms, E., Chu, T., Henrion, G., and Strickland, S. (2000). The only function of Grauzone required for Drosophila oocyte meiosis is transcriptional activation of the cortex gene. Genetics 155, 1831-1839. Jones, S., and Thornton, J. (1996). Principles of protein-protein interaction. Proc. Natl. Acad. Sci. USA 93, 13-20.

Klug, A., and Schwabe, J. (1995). Protein motifs 5: zinc fingers. FASEB J. 9, 597-604.

Krishna, S., Indraneel, M., and Grishin, N. (2003). Structural classification of zinc fingers: survey and summary. Nucleic Acids Res. 31, 532-550.

Laity, J., Lee, M., and Wright, P. (2001). Zinc finger proteins: new insights into structural and functional diversity. Curr. Opin. Struct. Biol. 11, 39-46.

Lander, E., Linton, L.M., Birren, B., Nusbaum, C., Zody, M.C., Baldwin, J., Devon, K., Dewar, K., Doyle, M., FitzHugh, W., et al. (2001). Initial sequencing and analysis of the human genome. Nature 409, 860-921.

Laskowski, R., MacArthur, M., Moss, D., and Thornton, J. (1993). PROCHECK: a program to check the stereochemical quality of protein structures. J. Appl. Crystallogr. 26, 283-291.

Lespinet, O.W., YI. Koonin, EV., and Aravind, L. (2002). The role of lineage-specific gene family expansion in the evolution of eukaryotes. Genome Res. 12, 1048-1059. 
Looman, C., Abrink, M., Mark, C., and Hellman, L. (2002). KRAB zinc finger proteins: an analysis of the molecular mechanisms governing their increase in numbers and complexity during evolution. Mol. Biol. Evol. 19, 2118-2130.

Lorick, K., Jensen, J., Fang, S., Ong, A., Hatakeyama, S., and Weissman, A. (1999). RING fingers mediate ubiquitin-conjugating enzyme (E2)-dependent ubiquitination. Proc. Natl. Acad. Sci. USA 96, 1136411369.

Luisi, B., Xu, W., Otwinowski, Z., Freedman, L., Yamamoto, K., and Sigler, P. (1991). Crystallographic analysis of the interaction of the glucocorticoid receptor with DNA. Nature 352, 497-505.

Luzzati, V. (1952). Traitement statistique des erreurs dans la détermination des structures cristallines. Acta Crystallogr. A 5, 802-810.

Mackay, J., and Crossley, M. (1998). Zinc fingers are sticking together. Trends Biochem. Sci. 23, 1-4.

Margolin, J., Friedman, J., Meyer, W., Vissing, H., Thiesen, H., and Rauscher, F. (1994). Kruppel-associated boxes are potent transcriptional repression domains. Proc. Natl. Acad. Sci. USA 91, 4509-4513.

McCarty, A., Kleiger, G., Eisenberg, D., and Smale, T. (2003). Selective dimerization of a $\mathrm{C} 2 \mathrm{H} 2$ Zinc Finger Subfamily. Mol. Cell 11, 459-470.

Merritt, E., and Bacon, D.J. (1997). Raster3D: photorealistic molecular graphics. Methods Enzymol. 277, 505-524.

Miller, J., McLachlan, A., and Klug, A. (1985). Repetitive zinc-binding domains in the protein transcription factor IIIA from Xenopus oocytes. EMBO J. 4, 1609-1614.

Otwinowski, Z., and Minor, W. (1997). Processing of X-ray diffraction data collected in oscillation mode. Methods Enzymol. 276, 307-326.

Pavletich, N., and Pabo, C. (1991). Zinc finger-DNA recognition: crystal structure of a Zif268-DNA complex at $2.1 \AA ̊$. Science 252, 809-817.

Payre, F., Crozatier, M., and Vincent, A. (1994). Direct control of transcription of the Drosophila morphogen bicoid by the serendipity delta zinc finger protein, as revealed by in vivo analysis of a finger swap. Genes Dev. 8, 2718-2728.

Payre, F., Buono, P., Vanzo, N., and Vincent, A. (1997). Two types of zinc fingers are required for dimerization of the serendipity delta transcriptional activator. Mol. Cell. Biol. 17, 3137-3145.

Peng, H., Begg, G., Harper, S., Friedman, J., Speicher, D., and Rauscher, F. (2000). Biochemical analysis of the Kruppel-associated box (KRAB) transcriptional repression domain. J. Biol. Chem. 275, 18000-18010.

CCP4 (Collaborative Computational Project 4) (1994). The CCP4 suite: programs for protein crystallography. Acta Crystallogr. D 50, 760-763.

Rosenberg, H., Schroder, C., Preiss, A., Kienlin, A., Cote, S., Riede, I., and Jackle, H. (1986). Structural homology of the product of the Drosophila Krüppel gene with Xenopus transcription factor-IIIA. Nature 319, 336-339.

Ruez, C., Payre, F., and Vincent, A. (1998). Transcriptional control of Drosophila bicoid by Serendipity delta: cooperative binding sites, promoter context, and co-evolution. Mech. Dev. 78, 125-134.

Thompson, J., Higgins, D., and Gibson, T. (1994). CLUSTAL W: improving the sensitivity of progressive multiple sequence alignment through sequence weighting, position-specific gap penalties and weight matrix choice. Nucleic Acids Res. 22, 4673-4680.

Tjandra, N., Omichinski, J., Gronenborn, A., Clore, G., and Bax, A. (1997). Use of dipolar $1 \mathrm{H}-15 \mathrm{~N}$ and $1 \mathrm{H}-13 \mathrm{C}$ couplings in the structure determination of magnetically oriented macromolecules in solution. Nat. Struct. Biol. 4, 732-738.

Turk, D. (1996). MAIN 96: an interactive software for density modifications, model building, structure refinement and analysis. In Meeting of the International Union of Crystallography Macromolecular Macromolecular Computing School, P.E. Bourne and K. Watenpaugh, eds. (International Union of Crystallography).

Wolfe, S., Nekludova, L., and Pabo, C. (2000). DNA recognition by Cys2His2 zinc finger proteins. Annu. Rev. Biophys. Biomol. Struct. 29, 183-212.
Wyatt, P. (1993). Light scattering and the absolute characterisation of macromolecules. Anal. Chim. Acta 272, 1-40.

\section{Accession Numbers}

The structure has been submitted to the Protein Data Bank (http:// www.rscb.org/pdb) under accession number 1PZW. 


\section{General Discussion}

\section{Zinc Coordinating Protein Modules and Intermolecular Recognition}

Zinc ions are found in a plethora of proteins that belong to a variety of functional and structural classes (Krishna et al., 2003). Besides acting as co-factors in catalytic reactions, e.g. in certain proteases (Hernick and Fierke, 2005), zinc ions function to stabilize structural modules remote from catalytic centers and enable the formation of small and highly compact folds (Krishna et al., 2003). In the latter case, zinc ions contribute to the architectural integrity of protein modules which are often involved in intermolecular recognition (Laity et al., 2001). In this study, zinc ions have been identified in two unrelated classes of proteins (Chapter I, Supplementary Chapter IV). The first class is represented by the zinc finger associated domain (ZAD) of the Drosophila transcription factor Grauzone.

The ZAD constitute a $\sim 80$ amino acid sequence motif that is specific for insects and genes encoding the domain are expanded in their genomes (Chung et al., 2002). The ZAD of the Drosophila transcriptional activator Grauzone represents a prototype member of this insect specific domain class ( Harms et al., 2000; Chung et al., 2002). It has been demonstrated that the ZAD constitutes a protein-protein interaction module which enables dimerization of ZAD containing proteins in an antiparallel fashion (Supplementary Chapter IV). In the ZAD, the zinc ion is coordinated by a cluster of four Cys residues. This arrangement leads to the formation of a spherical and compact N-terminal portion of the domain from which a Cterminal $\alpha$-helix emanates. This $\alpha$-helix accommodates most of the conserved and hydrophobic amino acid residues required for dimerization (Supplementary Chapter IV). Although the ZAD has some architectural similarities with the large group of the so-called treble clef zinc fingers (Grishin, 2001), it exhibits unique features which led to the definition of a novel protein domain family (Zf-AD, synonym ZAD; Pfam accession number PF07776). Since the ZAD is almost exclusively associated with transcription factors of the canonical $\mathrm{C} 2 \mathrm{H} 2$ zinc-finger type, it may contribute to the regulation of gene expression likely by mediating the assembly of distinct ZAD bearing proteins on clustered target sequences. 
A second zinc coordination site has been detected within the catalytic domain of Mnk2 (Chapter I). In this case, the zinc ion is coordinated by a Cys cluster located within an amino acid insertion upstream of helix $\alpha \mathrm{G}$ that is not found in other PKs. This insertion is phylogenetically conserved within the Mnk subfamily. A zinc coordination site as integral part of a PKD is unprecedented for this class of enzymes. The zinc site shapes a C-terminal extension of the enzyme. This extension is present in all protein kinases but absent in, for example, many metabolite kinases (Kannan and Neuwald, 2005). The C-terminal part of PKs and in particular helix $\alpha G$ has therefore been proposed to provide a scaffold for proteinprotein recognition (Kannan and Neuwald, 2005; Kim et al., 2005)\}. Hence, although the precise function of the zinc containing structural motifs of Mnks remains elusive, it is likely involved in the binding to substrates and/or regulator proteins. Taken together, the results obtained for both the Mnks and the ZAD highlight the significance of zinc ions in shaping protein domains and provide novel examples of zinc containing protein-protein recognition motifs.

\section{Mechanistic Plasticity of Biological Catalysts: Lessons from Mnk1/2 and NADS}

The structural studies on Mnk subfamily members and on the ecoNADS (see Chapters I, II and Supplementary Chapter III) provide different snapshots of highly dynamic enzymes which illustrate mechanistic rearrangements of their functional elements. The ecoNADS in complex with different substrates and products, as well as the three structures of Mnk subfamily members, permit to draw a dynamic picture of the respective enzymes. The plasticity of Mnk subfamily members is posed by alternative conformations of closely related isoforms and active site mutants, whereas the structural changes of NADS are depending on the association with different substrates and products. Binding of ATP to NADS is accomplished through an induced fit mechanism that leads to the reorganization of a short loop and amino acid side chains. The NAD site of NADS adopts alternative conformations depending on whether the substrate NAAD or the product NAD is bound. This structural rearrangement is accompanied by a reorientation of the ligand itself and reflects necessities which enable, on one hand, the catalytic reaction, and on the other hand, product release (Supplementary Chapter III). 
The crystal structures of the PKDs of Mnk1, Mnk2 and the active site mutant Mnk2 ${ }^{\mathrm{D} 228 \mathrm{G}}$ reveal a dynamic behavior which distinguishes the Mnk subfamily from other PKs. The dynamic behavior is manifested in pronounced conformational differences of the isoforms Mnk1 and Mnk2 despite their almost identical amino acid sequence. Mnk1 and Mnk2 vary with respect to the relative orientation of structural subdomains ( $\mathrm{N}$ - and $\mathrm{C}$-terminal lobe), the conformation of the activation segment and the side chain orientation of active site residues (Chapter I and II). These conformational differences can be attributed to distinct snapshots of different states of activity which are common to members of the Mnk subfamily of PKs. The structure of the Mnk2 $2^{\mathrm{D} 228 \mathrm{G}}$ mutant demonstrates how a single amino-acid substitution affects the stability of the catalytically relevant magnesium binding loop of the PK domain. In conclusion, the results suggest a novel regulatory mechanism that includes a gradual interconversion of the conformational states depicted in the structures of Mnk1, Mnk2 and $\mathrm{Mnk} 2^{\mathrm{D} 228 \mathrm{G}}$.

\section{The Structures of Mnk1 and Mnk2 Reveal a Novel Mechanism of Protein Kinase Regulation}

Although the more than 500 human PKs exhibit a very similar overall architecture, individual members function in distinct biological pathways and often respond to specific signaling events ( Krebs, 1983; Manning et al., 2002; Kannan and Neuwald, 2005). At the structural level, this specificity is achieved by a diverse set of regulatory mechanisms which distinguish individual PKs and adjust their activities (Huse and Kuriyan, 2002). As a consequence, most PKs exhibit intricate structural features which are specific and facilitate their differential regulation. Such features constitute adjoining regulatory domains, appropriate recognition sites for such domains and for regulatory subunits as well as specific phosphorylation sites.

The active site and, in particular, the activation segment, frame structural hot spots at which regulatory events converge (Nolen et al., 2004). Subtle structural changes affecting these regions of a PKD may be a prerequisite for productive substrate peptide and/or ATP binding. The activation of protein kinases is therefore accompanied by dynamic events which alter the conformation of structural elements reaching from individual amino acids to 
structural domains. The activation segment, for example, is highly flexible and often subjected to pronounced structural changes as a result of phosphorylation events (Nolen et al., 2004). In addition, regulatory events may influence the orientation of the $\mathrm{N}$ - and $\mathrm{C}$-terminal lobes of the PKD relative to each other and, in particular, the conformation of the regulatory helix $\alpha \mathrm{C}$. These rearrangements affect the chemical environment of the ATP binding site and, in turn, alter its accessibility and the catalytic activity (Huse and Kuriyan, 2002). Such mechanisms are pursued, for example, by the binding of cyclins to helix $\alpha \mathrm{C}$ at the N-lobe of CDKs (for CDKs this helix is also called PSTAIRE helix). The structure of cyclinA bound to CDK2 revealed that cyclinA binding induces a combination of rotating and translating movements of the $\alpha$ C-helix (Jeffrey et al., 1995). This way, the binding displaces certain backbone atoms by as much as $8.5 \AA$ when compared to the cyclinA free structure of CDK2 (Jeffrey et al., 1995). As a result of this movement, active site residues are positioned in a productive configuration and the kinase adopts an active conformation. A further principle of PK regulation is implemented by autoinhibiting domains located outside the core of the PKD. Autoinhibiting domains can obstruct PK function by acting as substrate mimics which occupy the substrate binding site as observed with Twitchin (Kobe et al., 1996), or by distorting the productive configuration of actives site elements as observed with CaMKI (Goldberg et al., 1996). The juxtamembrane (JM) domain constitutes a further regulatory element which is found in a subset of receptor protein tyrosine kinases (RTK). The JM domain links the transmembrane helix to the catalytic PKD and functions as an autoinhibitory domain in members of the platelet-derived growth factor receptor (PDGFR) family of RTKs such as c-KIT and FLT3 (Arkin and Wells, 2004; Griffith et al., 2004; Hubbard, 2004). The release of the JM domainmediated autoinhibition in c-KIT and FLT3 is achieved through phosphorylation of conserved tyrosine residue located within the JM domain (Hubbard, 2004).

The structures of Mnk subfamily members reveal a novel autoinhibitory mechanism of PK regulation (Chapter II). But Mnks do not contain autoinhibitory regions outside the core of the PKD such as a JM domain (Parra-Palau et al., 2003; Scheper et al., 2003). Hence, Mnks employ elements to achieve autoinhibition which are integral parts of the PKD. However, to stay inactive, Mnks employ a 'molecular trick' which strikingly similar to the JM domain mediated autoinhibition of PDGFRs. Both classes of PKs obstruct ATP binding by sterically interfering with the productive DFG/D-in conformation and induce the inhibitory DFG/D-out 
conformation. This rearrangement is achieved by inserting hydrophobic amino acid residues into a structural region referred to as $\mathrm{DFG} / \mathrm{D}$-in pocket. The origin of these inserted residues, however, differs between PDGFRs and Mnks. In contrast to c-KIT and FLT3 where inhibiting amino acid residues emanate form the JM domain, the Mnks make use of a specific Phe from the $\alpha \mathrm{EF} / \alpha \mathrm{F}$ loop of the extended and structurally rearranged activation segment. Hence, PDGFRs and Mnks employ an analogous strategy to prevent constitutive PK activity. Albeit, the structural elements used for this purpose are of different structural origin.

The occupation of a novel binding site by the activation segment of Mnk1 constitutes the prerequisite for the induction of DFG/D-out. This repositioning of the activation segment has further structural consequences: By binding to residues from helix $\alpha \mathrm{C}$ that otherwise interact with phosphor residues the helix $\alpha \mathrm{C}$ and the remainder $\mathrm{N}$-terminal lobe is reoriented and the interlobal cleft is closed (see Chapter II for details). Both series of structural changes, i.e. the induction of DFG/D-out and the N-lobe reorientation, crosstalk and enhance each other by intramolecular contacts at the ATP binding site. Notably, the structural data derived from both, the Mnk2 and the Mnk2 $2^{\mathrm{D} 28 \mathrm{G}}$ active site mutant, display a partial, but yet incomplete, release from autoinhibition (Chapter I). Due to the high degree of conservation within their primary sequences it is likely that Mnk1 and Mnk2 behave similarly on the molecular level. Hence, I assume that the different conformational states as seen for Mnk1 and Mnk2 reflect alternative states which can be adopted by each one of the two isoforms. The combination of the results enables to depict the mechanistic events that accompany activation of Mnk subfamily members. The resulting model illustrates a novel mechanism of protein kinase regulation and expands the functional roles of the activation segment and in particular of the helix $\alpha E F$ and the $\alpha E F / \alpha F$ loop. The Mnk structures also highlight the significance of the DFG/D motif for PK regulation (see discussion of Chapter II for details of the model). The study on Mnk regulation is therefore relevant for mechanistic and regulatory aspects of PKs in general (Huse and Kuriyan, 2002; Nolen et al., 2004). 


\section{The Structures of ZAD, NADS and Mnk1/2 are Relevant for Structure Guided Drug Design}

'The first protein structures revealed wonderful new faces of nature, but they did not help to cure anyone'. This statement, made by Max Perutz in 1992, illustrates that structural biology is, at least in part, driven by the desire to aid medical research (Perutz, 1992). The availability of an overwhelming amount of structural data and technology advancements on several levels may now indeed 'help to cure' since structure-guided drug design has turned into a commonly recognized discipline which is widely applied by the pharmaceutical industry (Nienaber et al., 2000; Williams et al., 2005). The authors of one of the first protein/drug costructure, which is the bacterial enzyme dihydrofolate reductase in complex with methotrexate, set the trend by saying: 'Perhaps it is worth noting here that the availability of the kind of detailed structural information ... provides an opportunity for rational drug design ... .' (Matthews et al., 1977). This prediction had soon been met. Among the first examples of successful applications of structure-guided drug design studies count the development of a potent agent against the influenza virus (von Itzstein et al., 1993) and inhibitors for the HIV protease (Ridky and Leis, 1995). Likewise, the proteins studied in the course of this PhD-work constitute potential drug targets or are related to pharmaceutically relevant proteins, respectively. Inasmuch, their crystal structures may provide valuable templates for structure guided drug design efforts. The availability of inhibitors against the studied proteins could, in addition to their medical applications, also potentiate basic functional understanding on the organismic and the tissue culture level.

PKs are implicated in many severe diseases. However, they have initially been considered to be 'not drugable' because of the strong conservation of their ATP binding site and the high intracellular ATP concentration (Cohen, 2002). Nevertheless, the first selective PK inhibitor was launched in 2001 (O'Dwyer and Druker, 2000). Subsequently, PKs turned into a major target of drug development programs (Noble et al., 2004). As a result, several additional PK inhibitors were launched and the pipelines of the pharmaceutical industries are filled with promising candidates (Vieth et al., 2005). Currently, protein kinases constitute the most important drug target in conjunction with the G-protein coupled receptors and investigators make tremendous use of structural data when designing and optimizing small molecule inhibitors (Cohen et al., 2005; Gill et al., 2005). The majority of small-molecule PK inhibitors achieve inactivation through direct binding to the ATP site. However, the desire for 
selectivity encouraged the search for alternative inhibitory strategies. Consequently, novel agents have been found comprising monoclonal antibodies and small molecules which recognize binding sites remote from the ATP binding pocket (O'Loghlen et al., 2004; Vieth et al., 2005).

The crystal structures of Mnk1 (Chapter II) and Mnk2 (Chapter I) suggest novel strategies of PK inhibitor development (Vieth et al., 2004). The autoinhibited conformation of Mnk1 and Mnk2 as well as the presence of Mnk-specific residues at the active site, suggest distinct target sites for specific Mnk inhibition and for PK inhibition in general. This conclusion is based on two major observations. Firstly, the autoinhibitory DFG/D-out conformation of the magnesium binding loop resembles a conformation observed in the MAPK p38 which was induced by the inhibitor BIRB-796 (Pargellis et al., 2002). The mode of inhibition seen in the p38/BIRB-796 complex indicates the presence of a novel allosteric binding site which causes indirect inhibition by stabilizing DFG/D-out (Pargellis et al., 2002). This allosteric binding site is structurally less conserved than the actual ATP binding site and may therefore result in an improved selectivity of the corresponding inhibitors (Mol et al., 2004; Noble et al., 2004). Since Mnk1 and Mnk2 adopt DFG/D-out passively, they appear well suited for allosteric inhibition. This assumption is supported by the recent observation that the allosteric p38 inhibitor BIRB-769 binds to Mnk2 in vitro (Fabian et al., 2005). Furthermore, the occurrence of DFD instead of the canonical DFG assigns the chemical distinctiveness to the relevant binding site and could benefit selectivity. A second strategy of PK inhibition can be deduced from the induction of autoinhibition through the structurally rearranged activation segment as seen in the Mnk1 structure. As described above, the occupation of the DFG/D-in pocket through a hydrophobic residue induces DFG/D-out and thereby switches the PK off. Hence, the development of agents which target the DFG/D-in pocket may represent a novel strategy for the design and screening for protein kinase inhibitors. Peptidomimetics resembling the autoinhibitory elements encoded by the $\alpha$ EF helix and the $\alpha E F-\alpha F$ loop region may represent a suitable starting point for such endeavors.

The second type of enzyme under study, the ecoNADS, is likewise of relevance for structure guided drug design. With the availability of structural data for the ecoNADS phylogenetic structure comparisons with the corresponding enzyme of pathogenic bacteria are made possible. Namely, the NADS of Bacillus anthracis is targeted for the development of agents combating anthrax (Sutherland, 2003). Therefore, the availability of the crystal 
structure of various forms of the Escherischia coli NADS provided in Supplementary Chapter III and its detailed comparison to previously investigated Bacillus subtilis NADS structures can help to optimize inhibitory compounds by potentiating the selectivity (Supplementary Chapter III).

Traditionally, the small-molecule binding sites of enzymes and receptors are targeted by drug design efforts (Cochran, 2000). However, protein-protein recognition surfaces are often chemically more distinct and, thus, receive an increasing attention in the course of drug design efforts (Arkin and Wells, 2004; Sillerud and Larson, 2005). The insect-specific zincfinger associated domain (ZAD) represents such a potentially drugable protein-protein recognition site (Supplementary Chapter III). The ZAD exhibits a unique fold and amino acid substitutions wihin the ZAD of certain genes result in lethality (Crozatier et al., 1992; Gaszner et al., 1999). Furthermore, the analysis of the genomic sequence of humans and other vertebrates revealed that the ZAD is missing in the respective genomes (Chung et al., 2002). This finding is consistent with the assumption that drugs specifically targeting the ZAD would not be harmful to vertebrates including humans. Hence, the ZAD constitutes a molecular scaffold which could potentially be used to develop insecticides. One of the potential targets of such compounds could be the transmitter of malaria Anopheles gambiae (Supplementary Chapter IV). 


\section{Bibliography}

Adams, J. A. (2001). Kinetic and catalytic mechanisms of protein kinases. Chem Rev 101, 2271-2290.

Allemand, E., Guil, S., Myers, M., Moscat, J., Caceres, J. F., and Krainer, A. R. (2005). Regulation of heterogenous nuclear ribonucleoprotein A1 transport by phosphorylation in cells stressed by osmotic shock. Proc Natl Acad Sci U S A 102, 3605-3610.

Arkin, M. R., and Wells, J. A. (2004). Small-molecule inhibitors of protein-protein interactions: progressing towards the dream. Nat Rev Drug Discov 3, 301-317.

Arquier, N., Bourouis, M., Colombani, J., and Leopold, P. (2005). Drosophila Lk6 kinase controls phosphorylation of eukaryotic translation initiation factor $4 \mathrm{E}$ and promotes normal growth and development. Curr Biol 15, 19-23.

Bellon, S., Fitzgibbon, M. J., Fox, T., Hsiao, H. M., and Wilson, K. P. (1999). The structure of phosphorylated p38gamma is monomeric and reveals a conserved activation-loop conformation. Structure Fold Des 7, 1057-1065.

Brown, N. R., Noble, M. E., Endicott, J. A., and Johnson, L. N. (1999). The structural basis for specificity of substrate and recruitment peptides for cyclin-dependent kinases. Nat Cell Biol 1, 438-443.

Buxade, M., Parra, J. L., Rousseau, S., Shpiro, N., Marquez, R., Morrice, N., Bain, J., Espel, E., and Proud, C. G. (2005). The Mnks Are Novel Components in the Control of TNFalpha Biosynthesis and Phosphorylate and Regulate hnRNP A1. Immunity 23, 177-189.

Chung, H. R., Schafer, U., Jackle, H., and Bohm, S. (2002). Genomic expansion and clustering of ZAD-containing C2H2 zinc-finger genes in Drosophila. EMBO Rep 3, 1158-1162.

Cochran, A. G. (2000). Antagonists of protein-protein interactions. Chem Biol 7, R85-94.

Cohen, M. S., Zhang, C., Shokat, K. M., and Taunton, J. (2005). Structural bioinformaticsbased design of selective, irreversible kinase inhibitors. Science 308, 1318-1321. 
Cohen, P. (2002). Protein kinases--the major drug targets of the twenty-first century? Nat Rev Drug Discov 1, 309-315.

Crozatier, M., Kongsuwan, K., Ferrer, P., Merriam, J. R., Lengyel, J. A., and Vincent, A. (1992). Single amino acid exchanges in separate domains of the Drosophila serendipity delta zinc finger protein cause embryonic and sex biased lethality. Genetics $131,905-$ 916.

Duncan, R. F., Peterson, H., and Sevanian, A. (2005). Signal transduction pathways leading to increased eIF4E phosphorylation caused by oxidative stress. Free Radic Biol Med 38, 631-643.

Fabian, M. A., Biggs, W. H., Treiber, D. K., Atteridge, C. E., Azimioara, M. D., Benedetti, M. G., Carter, T. A., Ciceri, P., Edeen, P. T., Floyd, M., et al. (2005). A small moleculekinase interaction map for clinical kinase inhibitors. Nat Biotechnol, 329-336.

Feynman, R. P., Leighton, R. B., and Sands, M. (1963). The Feynman Lectures on Physics. Addison-Wesley.

Fischer, E. H., and Krebs, E. G. (1955). Conversion of phosphorylase b to phosphorylase a in muscle extracts. J Biol Chem 216, 121-132.

Fornerod, M., Ohno, M., Yoshida, M., and Mattaj, I. W. (1997). CRM1 is an export receptor for leucine-rich nuclear export signals. Cell 90, 1051-1060.

Fukunaga, R., and Hunter, T. (1997). MNK1, a new MAP kinase-activated protein kinase, isolated by a novel expression screening method for identifying protein kinase substrates. Embo J 16, 1921-1933.

Gaszner, M., Vazquez, J., and Schedl, P. (1999). The Zw5 protein, a component of the scs chromatin domain boundary, is able to block enhancer-promoter interaction. Genes Dev 13, 2098-2107.

Gebauer, F., and Hentze, M. W. (2004). Molecular mechanisms of translational control. Nat Rev Mol Cell Biol 5, 827-835.

Gill, A., Cleasby, A., and Jhoti, H. (2005). The discovery of novel protein kinase inhibitors by using fragment-based high-throughput x-ray crystallography. Chembiochem 6, 506512. 
Goldberg, J., Nairn, A. C., and Kuriyan, J. (1996). Structural basis for the autoinhibition of calcium/calmodulin-dependent protein kinase I. Cell 84, 875-887.

Griffith, J., Black, J., Faerman, C., Swenson, L., Wynn, M., Lu, F., Lippke, J., and Saxena, K. (2004). The structural basis for autoinhibition of FLT3 by the juxtamembrane domain. Mol Cell 13, 169-178.

Grishin, N. V. (2001). Treble clef finger--a functionally diverse zinc-binding structural motif. Nucleic Acids Res 29, 1703-1714.

Hanks, S. K. (2003). Genomic analysis of the eukaryotic protein kinase superfamily: a perspective. Genome Biol 4, 111.

Hanks, S. K., Quinn, A. M., and Hunter, T. (1988). The protein kinase family: conserved features and deduced phylogeny of the catalytic domains. Science 241, 42-52.

Harms, E., Chu, T., Henrion, G., and Strickland, S. (2000). The only function of Grauzone required for Drosophila oocyte meiosis is transcriptional activation of the cortex gene. Genetics 155, 1831-1839.

Hart, J. C., Hillier, I. H., Burton, N. A., and Sheppard, D. W. (1998). An Alternative Role of the Conserved Asp Residue in Phosphoryl Transfer Reactions. J Am Chem Soc 120, 13535-13536.

Hefner, Y., Borsch-Haubold, A. G., Murakami, M., Wilde, J. I., Pasquet, S., Schieltz, D., Ghomashchi, F., Yates, J. R., 3rd, Armstrong, C. G., Paterson, A., et al. (2000). Serine 727 phosphorylation and activation of cytosolic phospholipase A2 by MNK1-related protein kinases. J Biol Chem 275, 37542-37551.

Hernick, M., and Fierke, C. A. (2005). Zinc hydrolases: the mechanisms of zinc-dependent deacetylases. Arch Biochem Biophys 433, 71-84.

Hubbard, S. R. (1997). Crystal structure of the activated insulin receptor tyrosine kinase in complex with peptide substrate and ATP analog. Embo J 16, 5572-5581.

Hubbard, S. R. (2004). Juxtamembrane autoinhibition in receptor tyrosine kinases. Nat Rev Mol Cell Biol 5, 464-471.

Huse, M., and Kuriyan, J. (2002). The conformational plasticity of protein kinases. Cell 109, 275-282. 
Jeffrey, P. D., Russo, A. A., Polyak, K., Gibbs, E., Hurwitz, J., Massague, J., and Pavletich, N. P. (1995). Mechanism of CDK activation revealed by the structure of a cyclinA-CDK2 complex. Nature 376, 313-320.

Johnson, L. N., Noble, M. E., and Owen, D. J. (1996). Active and inactive protein kinases: structural basis for regulation. Cell $85,149-158$.

Kannan, N., and Neuwald, A. F. (2005). Did protein kinase regulatory mechanisms evolve through elaboration of a simple structural component? J Mol Biol 351, 956-972.

Kim, C., Xuong, N. H., and Taylor, S. S. (2005). Crystal structure of a complex between the catalytic and regulatory (RIalpha) subunits of PKA. Science 307, 690-696.

Knighton, D. R., Zheng, J. H., Ten Eyck, L. F., Ashford, V. A., Xuong, N. H., Taylor, S. S., and Sowadski, J. M. (1991a). Crystal structure of the catalytic subunit of cyclic adenosine monophosphate-dependent protein kinase. Science 253, 407-414.

Knighton, D. R., Zheng, J. H., Ten Eyck, L. F., Xuong, N. H., Taylor, S. S., and Sowadski, J. M. (1991b). Structure of a peptide inhibitor bound to the catalytic subunit of cyclic adenosine monophosphate-dependent protein kinase. Science 253, 414-420.

Kobe, B., Heierhorst, J., Feil, S. C., Parker, M. W., Benian, G. M., Weiss, K. R., and Kemp, B. E. (1996). Giant protein kinases: domain interactions and structural basis of autoregulation. Embo J 15, 6810-6821.

Krebs, E. G. (1983). Historical perspectives on protein phosphorylation and a classification system for protein kinases. Philos Trans R Soc Lond B Biol Sci 302, 3-11.

Krebs, E. G., and Fischer, E. H. (1956). The phosphorylase b to a converting enzyme of rabbit skeletal muscle. Biochim Biophys Acta 20, 150-157.

Krebs, E. G., Kent, A. B., and Fischer, E. H. (1958). The muscle phosphorylase b kinase reaction. J Biol Chem 231, 73-83.

Krishna, S. S., Majumdar, I., and Grishin, N. V. (2003). Structural classification of zinc fingers: survey and summary. Nucleic Acids Res 31, 532-550.

Laity, J. H., Lee, B. M., and Wright, P. E. (2001). Zinc finger proteins: new insights into structural and functional diversity. Curr Opin Struct Biol 11, 39-46. 
Lander, E. S., Linton, L. M., Birren, B., Nusbaum, C., Zody, M. C., Baldwin, J., Devon, K., Dewar, K., Doyle, M., FitzHugh, W., et al. (2001). Initial sequencing and analysis of the human genome. Nature 409, 860-921.

Lowe, E. D., Noble, M. E., Skamnaki, V. T., Oikonomakos, N. G., Owen, D. J., and Johnson, L. N. (1997). The crystal structure of a phosphorylase kinase peptide substrate complex: kinase substrate recognition. Embo J 16, 6646-6658.

Manning, G., Whyte, D. B., Martinez, R., Hunter, T., and Sudarsanam, S. (2002). The protein kinase complement of the human genome. Science 298, 1912-1934.

Marcotrigiano, J., Gingras, A. C., Sonenberg, N., and Burley, S. K. (1997). Cocrystal structure of the messenger RNA 5' cap-binding protein (eIF4E) bound to 7-methyl-GDP. Cell $89,951-961$.

Matthews, D. A., Alden, R. A., Bolin, J. T., Freer, S. T., Hamlin, R., Xuong, N., Kraut, J., Poe, M., Williams, M., and Hoogsteen, K. (1977). Dihydrofolate reductase: x-ray structure of the binary complex with methotrexate. Science 197, 452-455.

McKendrick, L., Morley, S. J., Pain, V. M., Jagus, R., and Joshi, B. (2001). Phosphorylation of eukaryotic initiation factor $4 \mathrm{E}$ (eIF4E) at Ser209 is not required for protein synthesis in vitro and in vivo. Eur J Biochem 268, 5375-5385.

Mol, C. D., Fabbro, D., and Hosfield, D. J. (2004). Structural insights into the conformational selectivity of STI-571 and related kinase inhibitors. Curr Opin Drug Discov Devel 7, 639-648.

Morley, S. J., and Naegele, S. (2002). Phosphorylation of eukaryotic initiation factor (eIF) 4E is not required for de novo protein synthesis following recovery from hypertonic stress in human kidney cells. J Biol Chem 277, 32855-32859.

Nagar, B., Hantschel, O., Young, M. A., Scheffzek, K., Veach, D., Bornmann, W., Clarkson, B., Superti-Furga, G., and Kuriyan, J. (2003). Structural basis for the autoinhibition of c-Abl tyrosine kinase. Cell 112, 859-871.

Nienaber, V. L., Richardson, P. L., Klighofer, V., Bouska, J. J., Giranda, V. L., and Greer, J. (2000). Discovering novel ligands for macromolecules using X-ray crystallographic screening. Nat Biotechnol 18, 1105-1108. 
Nikolcheva, T., Pyronnet, S., Chou, S. Y., Sonenberg, N., Song, A., Clayberger, C., and Krensky, A. M. (2002). A translational rheostat for RFLAT-1 regulates RANTES expression in T lymphocytes. J Clin Invest 110, 119-126.

Noble, M. E., Endicott, J. A., and Johnson, L. N. (2004). Protein kinase inhibitors: insights into drug design from structure. Science 303, 1800-1805.

Nolen, B., Taylor, S., and Ghosh, G. (2004). Regulation of protein kinases; controlling activity through activation segment conformation. Mol Cell 15, 661-675.

O'Dwyer, M. E., and Druker, B. J. (2000). STI571: an inhibitor of the BCR-ABL tyrosine kinase for the treatment of chronic myelogenous leukaemia. Lancet Oncol 1, 207-211.

O'Loghlen, A., Gonzalez, V. M., Pineiro, D., Perez-Morgado, M. I., Salinas, M., and Martin, M. E. (2004). Identification and molecular characterization of Mnk1b, a splice variant of human MAP kinase-interacting kinase Mnk1. Exp Cell Res 299, 343-355.

Pargellis, C., Tong, L., Churchill, L., Cirillo, P. F., Gilmore, T., Graham, A. G., Grob, P. M., Hickey, E. R., Moss, N., Pav, S., and Regan, J. (2002). Inhibition of p38 MAP kinase by utilizing a novel allosteric binding site. Nat Struct Biol 9, 268-272.

Parra-Palau, J. L., Scheper, G. C., Wilson, M. L., and Proud, C. G. (2003). Features in the N and $\mathrm{C}$ termini of the MAPK-interacting kinase Mnk1 mediate its nucleocytoplasmic shuttling. J Biol Chem 278, 44197-44204.

Pawson, T., and Scott, J. D. (2005). Protein phosphorylation in signaling--50 years and counting. Trends Biochem Sci 30, 286-290.

Perutz, M. F. (1992). Protein Structure. WH Freeman and Company.

Preiss, T., and Hentze, M. W. (2003). Starting the protein synthesis machine: eukaryotic translation initiation. Bioessays 25, 1201-1211.

Proud, C. G. (2005). The eukaryotic initiation factor 4E-binding proteins and apoptosis. Cell Death Differ 12, 541-546.

Pyronnet, S., Imataka, H., Gingras, A. C., Fukunaga, R., Hunter, T., and Sonenberg, N. (1999). Human eukaryotic translation initiation factor 4G (eIF4G) recruits mnk1 to phosphorylate eIF4E. Embo J 18, 270-279. 
Reiling, J. H., Doepfner, K. T., Hafen, E., and Stocker, H. (2005). Diet-dependent effects of the Drosophila Mnk1/Mnk2 homolog Lk6 on growth via eIF4E. Curr Biol 15, 24-30.

Ridky, T., and Leis, J. (1995). Development of drug resistance to HIV-1 protease inhibitors. J Biol Chem 270, 29621-29623.

Rizzi, M., Bolognesi, M., and Coda, A. (1998). A novel deamido-NAD+-binding site revealed by the trapped NAD-adenylate intermediate in the NAD+ synthetase structure. Structure 6, 1129-1140.

Rizzi, M., Nessi, C., Mattevi, A., Coda, A., Bolognesi, M., and Galizzi, A. (1996). Crystal structure of NH3-dependent NAD+ synthetase from Bacillus subtilis. Embo J 15, $5125-5134$.

Sachs, A. B. (2000). Cell cycle-dependent translation initiation: IRES elements prevail. Cell $101,243-245$.

Scheper, G. C., Morrice, N. A., Kleijn, M., and Proud, C. G. (2001). The mitogen-activated protein kinase signal-integrating kinase Mnk2 is a eukaryotic initiation factor 4E kinase with high levels of basal activity in mammalian cells. Mol Cell Biol 21, 743 754.

Scheper, G. C., Parra, J. L., Wilson, M., Van Kollenburg, B., Vertegaal, A. C., Han, Z. G., and Proud, C. G. (2003). The N and C termini of the splice variants of the human mitogenactivated protein kinase-interacting kinase Mnk2 determine activity and localization. Mol Cell Biol 23, 5692-5705.

Scheper, G. C., and Proud, C. G. (2002). Does phosphorylation of the cap-binding protein eIF4E play a role in translation initiation? Eur J Biochem 269, 5350-5359.

Scheper, G. C., van Kollenburg, B., Hu, J., Luo, Y., Goss, D. J., and Proud, C. G. (2002). Phosphorylation of eukaryotic initiation factor $4 \mathrm{E}$ markedly reduces its affinity for capped mRNA. J Biol Chem 277, 3303-3309.

Sillerud, L. O., and Larson, R. S. (2005). Design and structure of peptide and peptidomimetic antagonists of protein-protein interaction. Curr Protein Pept Sci 6, 151-169.

Slentz-Kesler, K., Moore, J. T., Lombard, M., Zhang, J., Hollingsworth, R., and Weiner, M. P. (2000). Identification of the human Mnk2 gene (MKNK2) through protein interaction with estrogen receptor beta. Genomics 69, 63-71. 
Sonenberg, N., and Gingras, A. C. (1998). The mRNA 5' cap-binding protein eIF4E and control of cell growth. Curr Opin Cell Biol 10, 268-275.

Sutherland, E. W., Jr., and Wosilait, W. D. (1955). Inactivation and activation of liver phosphorylase. Nature 175, 169-170.

Sutherland, S. (2003). New antibiotics for anthrax? Drug Discov Today 8, 335-336.

Taylor, S. S., and Radzio-Andzelm, E. (1994). Three protein kinase structures define a common motif. Structure 2, 345-355.

Ueda, T., Watanabe-Fukunaga, R., Fukuyama, H., Nagata, S., and Fukunaga, R. (2004). Mnk2 and Mnk1 are essential for constitutive and inducible phosphorylation of eukaryotic initiation factor 4E but not for cell growth or development. Mol Cell Biol 24, 65396549.

Vieth, M., Higgs, R. E., Robertson, D. H., Shapiro, M., Gragg, E. A., and Hemmerle, H. (2004). Kinomics-structural biology and chemogenomics of kinase inhibitors and targets. Biochim Biophys Acta 1697, 243-257.

Vieth, M., Sutherland, J. J., Robertson, D. H., and Campbell, R. M. (2005). Kinomics: characterizing the therapeutically validated kinase space. Drug Discov Today 10, 839846.

von Itzstein, M., Wu, W. Y., Kok, G. B., Pegg, M. S., Dyason, J. C., Jin, B., Van Phan, T., Smythe, M. L., White, H. F., Oliver, S. W., and et al. (1993). Rational design of potent sialidase-based inhibitors of influenza virus replication. Nature 363, 418-423.

Walsh, D., and Mohr, I. (2004). Phosphorylation of eIF4E by Mnk-1 enhances HSV-1 translation and replication in quiescent cells. Genes Dev 18, 660-672.

Waskiewicz, A. J., Flynn, A., Proud, C. G., and Cooper, J. A. (1997). Mitogen-activated protein kinases activate the serine/threonine kinases Mnk1 and Mnk2. Embo J 16, 1909-1920.

Waskiewicz, A. J., Johnson, J. C., Penn, B., Mahalingam, M., Kimball, S. R., and Cooper, J. A. (1999). Phosphorylation of the cap-binding protein eukaryotic translation initiation factor 4E by protein kinase Mnk1 in vivo. Mol Cell Biol 19, 1871-1880.

Williams, S. P., Kuyper, L. F., and Pearce, K. H. (2005). Recent applications of protein crystallography and structure-guided drug design. Curr Opin Chem Biol 9, 371-380. 
Yang, J., Cron, P., Good, V. M., Thompson, V., Hemmings, B. A., and Barford, D. (2002). Crystal structure of an activated Akt/protein kinase B ternary complex with GSK3peptide and AMP-PNP. Nat Struct Biol 9, 940-944.

Zuberek, J., Wyslouch-Cieszynska, A., Niedzwiecka, A., Dadlez, M., Stepinski, J., Augustyniak, W., Gingras, A. C., Zhang, Z., Burley, S. K., Sonenberg, N., et al. (2003). Phosphorylation of eIF4E attenuates its interaction with mRNA 5' cap analogs by electrostatic repulsion: intein-mediated protein ligation strategy to obtain phosphorylated protein. Rna 9, 52-61. 


\section{Abbreviations}

ATP

CAMK

CDK

C-loop

DAPK

DFG

eIF

ERK

hnRNPA1

IRK

JM

MAPK

MAPKAP

Mnk

mRNA

NAAD

NAD

NADS

PDGFR

PK

PKD

RTK

TNF- $\alpha$

tRNA

UTR

VEGF-R

ZAD adenosine tri-phosphate

$\mathrm{Ca}^{2+}$ - calmodulin modulated protein kinase

cyclin dependent kinase

catalytic loop

death associated protein kinase

Asp-Phe-Gly tripeptide motif

eukaryotic initiation factor

extracellular signal regulated kinase

heterogenous nuclear ribonucleoprotein A1

insulin receptor kinase

juxtamembrane

mitogen activated protein kinase

MAPK activated protein kinase

MAPK interacting protein kinase

messenger RNA

nicotinic acid adenosine dinucleotide

nicotinamide adenosine dinucleotide

NAD synthetase

platelet-derived growth factor receptor

protein kinase

protein kinase domain

receptor tyrosine kinase

tumor necrosis factor alpha

transfer RNA

untranslated region

vascular endothelial growth factor receptor

zinc finger associated domain 


\section{Abbreviations - Amino Acids}

\begin{tabular}{|c|c|c|}
\hline Alanine & Ala & A \\
\hline Arginine & Arg & $\mathrm{R}$ \\
\hline Asparagine & Asn & $\mathrm{N}$ \\
\hline Aspartic acid & Asp & $\mathrm{D}$ \\
\hline Cysteine & Cys & $\mathrm{C}$ \\
\hline Glutamic acid & Glu & $\mathrm{E}$ \\
\hline Glutamine & Gln & Q \\
\hline Glycine & Gly & G \\
\hline Histidine & His & $\mathrm{H}$ \\
\hline Isoleucine & Ile & $\mathrm{I}$ \\
\hline Leucine & Leu & $\mathrm{L}$ \\
\hline Lysine & Lys & $\mathrm{K}$ \\
\hline Methionine & Met & $\mathrm{M}$ \\
\hline Phenylalanine & Phe & $\mathrm{F}$ \\
\hline Proline & Pro & $\mathrm{P}$ \\
\hline Serine & Ser & $\mathrm{S}$ \\
\hline Threonine & Thr & $\mathrm{T}$ \\
\hline Tryptophane & $\operatorname{Trp}$ & W \\
\hline Tyrosine & Tyr & $\mathrm{Y}$ \\
\hline Valine & Val & V \\
\hline
\end{tabular}




\section{Acknowledgements}

I'd like to express my gratitude to Prof. Dr. Herbert Jäckle for his inspiring advice, continuous interest in my work and seemingly infinite support. I particularly appreciated that he gave me the freedom develop my personal scientific interest without restrictions. I would also like to thank all members of the 'Abteilung für Molekulare Entwicklungsbiologie' for helpful advice, discussions and reagents. In this respect I'm particularly grateful to Dr. Ralf Pflanz.

None of my projects would have been possible without the close collaboration with the 'Arbeitsgruppe Röntgenkristallographie' led by Dr. Markus Wahl. I enjoyed a tremendous support provided by Markus in most aspects of my biochemical and crystallographic endeavors. I also thank the other members of his group for advice, help with reagents and for tolerating me as a 'contaminant' in their lab. I'm particularly grateful to Catharina Netter who helped me with the initial crystallographic work and by setting up the crystallization facility. In addition, she was always available for critically assessing my manuscripts and ideas. I also thank Dr. Gleb Bourenkov (DESY) and Dr. Ehmke Pohl (SLS) for excellent beamline maintenance, help and discussions.

I thank Prof. Dr. Reinhard Jahn and Prof. Dr. Thomas R. Schneider as members of my PhD committee for their support, helpful comments and critical evaluation of my work. The efficient operation of the coordination office of the 'MolBio'-program led by Dr. Steffen Burkhardt facilitated numerous aspects of the 'PhD life'.

The NADS project would not have been impossible without the initial work performed by Dr. Andreas Humm and his colleagues. I'm very grateful that I have been allowed to use his data. I thank the Develogen AG and in particular Dr. Stefan Jäkel, Dr. Babette Aicher and Dr. Cord Dohrmann for providing purified Mnk proteins. It's been a very fruitful and congenial collaboration. For numerous instructive discussions particularly during the lunch breaks I thank Ben Harder, Patrick Müller and Ajaybabu V. Pobbati. I'm grateful to Dr. Jörg Kahle and Anja Uhmann for proofreading this manuscript and many aspects beyond the scientific life. 


\title{
History of Education
}

\author{
2003-2005 Doctoral Studies \\ Max-Planck-Institut für biophysikalische Chemie, Göttingen, Germany \\ $2003 \quad$ Master's of Science, Grade 1.0 \\ 2001 - 2003 Advanced Studies in Molecular Biology/Master's Project \\ $\mathrm{MSc} / \mathrm{PhD}$ Program 'Molecular Biology', Göttingen, Germany \\ 2000 - 2001 Advanced Studies in Molecular Biology \\ University of Manchester, Manchester, UK \\ $2000 \quad$ Pre-Diploma Biology, Grade 1.2 \\ 1998 - $2000 \quad$ Undergraduate Studies Biology \\ Friedrich Schiller University, Jena, Germany \\ 1997 - 1999 Undergraduate Studies Archeology \\ Friedrich Schiller University, Jena, Germany \\ 1996 \\ 1984 - 1996 \\ Abitur, Grade 1.7 \\ School Education \\ Talschule and Angergymnasium, Jena, GDR/Germany
}

\section{Original Publications}

Jauch R, Jäkel S, Netter C, Schreiter K, Aicher B, Jäckle H and Wahl MC. The MAPK Interacting Kinase (Mnk) Subfamily is Regulated via a Novel Autoinhibitory Mechanism. to be submitted.

Jauch R, Jäkel S, Netter C, Schreiter K, Aicher B, Jäckle H and Wahl MC. Structures of Mnk2 Reveal an Inhibitory Conformation and a Zinc-Binding Site. Structure.2005. In press.

Jauch R, Humm A, Huber R, Wahl MC. Structures of E. coli NAD Synthetase with Substrates and Product Reveal Mechanistic Rearrangements. J Biol Chem. 2005; 280: 15131 15140

Jauch R, Bourenkov GP, Chung HR, Urlaub H, Reidt U, Jackle H, Wahl MC. The zinc finger-associated domain of the Drosophila transcription factor grauzone is a novel zinccoordinating protein-protein interaction module. Structure (Camb). 2003;(11):1393-402.

\section{Patents}

Jauch R, Wahl MC et al. Crystallographic structure of Mnk2 and Mnk1.(EP 050198899.3). assignee: DeveloGen AG and Max-Planck-Gesellschaft. filing date 13.9.2005

Jauch R, Chung HR, Wahl MC. Method for obtaining agents against insects by using the structural coordinates of $Z_{A D}$ Grau. (EP 03017076). assignee: Max-Planck-Gesellschaft. filing date 28.07.2003 